\title{
Novel primers improve species delimitation in Cercospora
}

\author{
Mounes Bakhshi ${ }^{1}$, Mahdi Arzanlou², Asadollah Babai-ahari², Johannes Z. Groenewald ${ }^{3}$, and Pedro W. Crous ${ }^{3,4,5}$
}

${ }^{1}$ Department of Botany, Iranian Research Institute of Plant Protection, P.O. Box 19395-1454, Agricultural Research, Education and Extension Organization (AREEO), Tehran, Iran; corresponding author e-mail: mounesbakhshi@gmail.com

2Plant Protection Department, Faculty of Agriculture, University of Tabriz, P.O. Box 5166614766, Tabriz, Iran

${ }^{3}$ Westerdijk Fungal Biodiversity Institute, Uppsalalaan 8, 3584 CT Utrecht, The Netherlands

${ }^{4}$ Department of Genetics, Biochemistry and Microbiology, Forestry and Agricultural Biotechnology Institute, University of Pretoria, Pretoria, 0002 , South Africa

${ }^{5}$ Wageningen University and Research Centre (WUR), Laboratory of Phytopathology, Droevendaalsesteeg 1, 6708 PB Wageningen, The Netherlands

Abstract: The genus Cercospora includes many important plant pathogens that are commonly associated with leaf spot diseases on a wide range of cultivated and wild plant species. Due to the lack of useful morphological features and high levels of intraspecific variation, host plant association has long been a decisive criterion for species delimitation in Cercospora. Because several taxa have broader host ranges, reliance on host data in Cercospora taxonomy has proven problematic. Recent studies have revealed multi-gene DNA sequence data to be highly informative for species identification in Cercospora, especially when used in a concatenated alignment. In spite of this approach, however, several species complexes remained unresolved as no single gene proved informative enough to act as DNA barcoding locus for the genus. Therefore, the aims of the present study were firstly to improve species delimitation in the genus Cercospora by testing additional genes and primers on a broad set of species, and secondly to find the best DNA barcoding gene(s) for species delimitation. Novel primers were developed for tub2 and rpb2 to supplement previously published primers for these loci. To this end, 145 Cercospora isolates from the Iranian mycobiota together with 25 additional reference isolates preserved in the Westerdijk Fungal Biodiversity Institute were subjected to an eight-gene (ITS, tef1, actA, cmdA, his3, tub2, rpb2 and gapdh) analysis. Results from this study provided new insights into DNA barcoding in Cercospora, and revealed gapdh to be a promising gene for species delimitation when supplemented with $c m d A$, tef1 and tub2. The robust eight-gene phylogeny revealed several novel clades within the existing Cercospora species complexes, such as C. apii, C. armoraciae, C. beticola, C. cf. flagellaris and Cercospora sp. G. The C. apii s. lat. isolates are distributed over three clades, namely C. apii s. str., C. plantaginis and C. uwebrauniana sp. nov. The $C$. armoraciae s. lat. isolates are distributed over two clades, C. armoraciae s. str. and C. bizzozeriana. The C. beticola s. lat. isolates are distributed over two clades, namely C. beticola s. str. and C. gamsiana, which is newly described.
Key words:

Bar codes

biodiversity

Cercospora apii complex

host specificity

multi-gene phylogeny

new taxa

Article info: Submitted: 14 March 2018; Accepted: 11 September 2018; Published: 26 September 2018.

\section{INTRODUCTION}

Fungi belonging to the genus Cercospora (Mycosphaerellaceae, Capnodiales) are common etiological agents of leaf spots, but some also cause necrotic lesions on flowers, fruits, bracts, seeds and pedicels of many woody and herbaceous plants in a range of climates worldwide (Ellis 1976, Crous \& Braun 2003, Agrios 2005, Groenewald et al. 2013, Bakhshi et al. 2015a).

Cercospora is a species-rich genus of cercosporoid fungi that was established by Fresenius (1863) for passalora-like species with pluriseptate conidia. During the course of the next 100 years, the concept of Cercospora had been continuously widened (Saccardo 1880, Solheim 1930) and all kinds of superficially similar species, with or without conspicuous conidiogenous loci, with hyaline or pigmented conidia, formed singly or in chains, were assigned to this genus (Braun et al. 2013). In 1954, the genus was monographed by Chupp (1954), who treated 1419 Cercospora species while applying this broad generic concept. He also stated that species of Cercospora were generally host-specific and used this argument as the basis of formulating the concept that each plant host genus or family would have its own Cercospora species. The number of Cercospora species increased rapidly to more than 3000, which led Pollack (1987) to publish her annotated list of Cercospora names. Since the introduction of the genus, several attempts to split Cercospora s. lat. into smaller generic units have been made by applying a combination of characters such as conidiomatal structure, mycelium, conidiophores, conidiogenous cells, and conidia

\section{@ 2018 International Mycological Association}

You are free to share - to copy, distribute and transmit the work, under the following conditions:

Attribution: $\quad$ You must attribute the work in the manner specified by the author or licensor (but not in any way that suggests that they endorse you or your use of the work).

Non-commercial: $\quad$ You may not use this work for commercial purposes.

No derivative works: You may not alter, transform, or build upon this work.

For any reuse or distribution, you must make clear to others the license terms of this work, which can be found at http://creativecommons.org/licenses/by-nc-nd/3.0/legalcode. Any of the above conditions can be waived if you get permission from the copyright holder. Nothing in this license impairs or restricts the author's moral rights. 
(e.g. Deighton 1973, 1979, 1983, Ellis 1971, 1976, Braun 1995, 1998). Crous \& Braun (2003) published an annotated list of the names published in Cercospora and Passalora and used the structure of conidiogenous loci and hila as well as the absence or presence of pigmentation in conidiophores and conidia in their revision. They recognised 659 names in Cercospora, with a further 281 species names reduced to synonymy with $C$. apii $s$. lat., since they were morphologically not or barely distinguishable from C. apii s. str. Braun et al. (2013, 2014, 2015a, b, 2016) published a series of papers in a stepwise approach at plant family level in order to update the monograph of Cercospora and allied genera.

Scientific advances in DNA sequencing and supplementary software to store, share and compare the emerging molecular data have revolutionised the procedures underpinning the discovery and identification of fungal taxa, including the cercosporoid fungi (Crous \& Groenewald 2005, Groenewald et al. 2013, Bakhshi et al. 2015a, Nguanhom et al. 2015, Guatimosim et al. 2017). Numerous molecular studies of Cercospora species have been conducted based on ITS nrDNA data as well as multi-gene approaches (Stewart et al. 1999, Crous et al. 2000, 2004b, 2009a, b, Goodwin et al. 2001, Tessmann et al. 2001, Pretorius et al. 2003, Groenewald et al. 2005, 2006, 2013, Montenegro-Calderón et al. 2011, Bakhshi et al. 2012b, 2015a, Nguanhom et al. 2015, Soares et al. 2015, Albu et al. 2016, Guatimosim et al. 2017, Guillin et al. 2017). A comprehensive and detailed molecular examination of Cercospora s. str. based on a multi-locus DNA sequence dataset of five genomic loci including the ITS (ITS1, 5.8S nrRNA gene and ITS2), together with parts of four protein coding genes, viz. translation elongation factor 1-alpha (tef1), actin (actA), calmodulin ( $\mathrm{cmdA}$ ) and histone $\mathrm{H} 3$ (his3) was conducted by Groenewald et al. (2013). The main conclusion of this study was that $C$. apii s. lat. could not be confirmed as a plurivorous monophyletic species, and that several lineages originally referred to $C$. apii s. lat., or considered close to this complex based on morphology (Crous \& Braun 2003), were separated as distinct phylogenetic species. Hence, speciation within Cercospora s. str. is more complicated than formerly assumed, and far from being resolved. To date, multi-locus DNA sequence analyses combined with ecology, morphology and cultural characteristics, referred to as the Consolidated Species Concept (Quaedvlieg et al. 2014), proved the most effective method for the delimitation of Cercospora species (Groenewald et al. 2010, 2013).

At a higher taxonomic level, among the genera of cercosporoid fungi, the monophyly of Cercospora s. str. has until recently been tested based on phylogenetic association of taxa with the type species of Cercospora, C. apii (Groenewald et al. 2013, Bakhshi et al. 2015a, Braun \& Crous 2016). Bakhshi et al. (2015b) recovered some cercospora-like isolates from Ammi majus, and in their subsequent multi-gene phylogenetic study (28S nrDNA, ITS, actA, tef1 and his3), elucidated these isolates to represent a new genus, Neocercospora, clustering in a clade in Mycosphaerellaceae apart from Cercospora s. str., suggesting that cercospora-like morphologies are not necessarily part of a single monophyletic genus. This finding led to the conclusion that identification and descriptions of new cercospora-like taxa should be avoided without support of molecular sequence data, not only at species but also at generic level.

Species of Cercospora are known to be widely distributed, occurring on a broad range of plant hosts in many climate zones of Iran (Bakhshi et al. 2012, Hesami et al. 2012, Pirnia et al. 2012), where the biodiversity of the genus has recently received much attention (Bakhshi et al. 2015a, b). The most inclusive study was that of Bakhshi et al. (2015a), who compared 161 Cercospora isolates, recovered from 74 host species from Iran based on DNA sequence data of five genomic loci (ITS, tef1, actA, cmdA and his3), host, cultural, and morphological data, revealing a rich species diversity. However, the problem concerning species delimitation in Cercospora due to the high level of conservation among DNA sequences of commonly used loci, (i.e. ITS, tef1, act $A, c m d A$, and his3), could not be resolved. Furthermore, cryptic clades in several species complexes remained unresolved in the fivegene phylogenetic tree, for example C. apii, C. armoraciae, C. cf. flagellaris, and Cercospora sp. G (Groenewald et al. 2013, Bakhshi et al. 2015a). Therefore, the aim of the present study was to assess three additional potential candidate gene regions including the partial $\beta$-tubulin (tub2) gene, part of the second largest subunit of RNA-polymerase II (rpb2) gene, and part of the glyceraldehyde-3-phosphate dehydrogenase (gapdh) gene, in order to firstly generate an eight-gene DNA dataset to resolve cryptic taxa within these species complexes, and secondly to identify the best barcoding gene(s) for species resolution in Cercospora.

\section{MATERIAL AND METHODS}

\section{Specimens and isolates}

A total of 170 strains, including 145 previously identified as Cercospora species in Bakhshi et al. (2015a), as well as 25 other related strains formerly identified by Groenewald et al. (2013), were studied. Isolates used in this study (Table 1) are maintained in the collection of the Westerdijk Fungal Biodiversity Institute (CBS), Utrecht, The Netherlands, the working collection of Pedro Crous (CPC; housed at CBS), the culture collection of the Iranian Research Institute of Plant Protection (IRAN C), Tehran, Iran, and the culture collection of Tabriz University (CCTU), Tabriz, Iran. Type material of the new species recognized is preserved in the Fungal Herbarium of the Iranian Research Institute of Plant Protection (IRAN F).

\section{DNA extraction and PCR amplification}

DNA samples comprised those previously extracted by Bakhshi et al. (2015a) and Groenewald et al. (2013). Three additional partial nuclear genes were targeted for PCR amplification and sequencing, namely, glyceraldehyde-3phosphate dehydrogenase (gapdh), RNA polymerase II second largest subunit (rpb2), and $\beta$-tubulin (tub2), using corresponding primer sets (Table 2). PCR amplifications were performed in a total volume of $12.5 \mu \mathrm{L}$ on a GeneAmp PCR System 9700 (Applied Biosystems, Foster City, CA). The gapdh PCR mixture consisted of 5-10 ng genomic DNA, $1 \times$ PCR buffer (Bioline, London), $2 \mathrm{mM} \mathrm{MgCl}_{2}$ (Bioline), $50 \mu \mathrm{M}$ of each dNTP, $0.5 \mu \mathrm{L}$ BSA (10 mg/ml) (Promega, Madison, $\mathrm{WI}), 0.28 \mu \mathrm{M}$ of each primer and 0.5 units GoTaq ${ }^{\circledR}$ Flexi DNA 
Table 1. Collection details and GenBank accession numbers of isolates included in this study. Ex-type isolates and newly generated sequences are highlighted in bold.

\begin{tabular}{|c|c|c|c|c|c|c|c|c|c|c|c|c|c|}
\hline \multirow[t]{2}{*}{ Species } & \multirow{2}{*}{$\begin{array}{l}\text { Culture accession } \\
\text { number }(\mathbf{s})^{1}\end{array}$} & \multirow[t]{2}{*}{ Host } & \multirow[t]{2}{*}{ Host Family } & \multirow[t]{2}{*}{ Origion } & \multirow[t]{2}{*}{ Collector } & \multicolumn{8}{|c|}{ GenBank accession numbers ${ }^{2}$} \\
\hline & & & & & & ITS & tef1 & act $A$ & $c m d A$ & his3 & tub2 & rpb2 & gapdh \\
\hline \multirow[t]{7}{*}{ Cercospora althaeina } & CCTU 1028 & Althaea rosea & Malvaceae & $\begin{array}{l}\text { Iran, Guilan, } \\
\text { Sowme'eh Sara }\end{array}$ & M. Bakhshi & KJ886394 & KJ886233 & KJ885911 & KJ885750 & KJ886072 & MH496336 & MH511833 & MH496166 \\
\hline & CCTU 1001 & Althaea rosea & Malvaceae & Iran, Guilan, Talesh & M. Bakhshi & KJ886392 & KJ886231 & KJ885909 & KJ885748 & KJ886070 & MH496337 & MH511834 & MH496167 \\
\hline & CCTU 1026 & Althaea rosea & Malvaceae & Iran, Guilan, Talesh & M. Bakhshi & KJ886393 & KJ886232 & KJ885910 & KJ885749 & KJ886071 & MH496338 & MH511835 & MH496168 \\
\hline & CCTU 1152 & Althaea rosea & Malvaceae & Iran, Guilan, Talesh & M. Bakhshi & KJ886396 & KJ886235 & KJ885913 & KJ885752 & KJ886074 & MH496339 & MH511836 & MH496169 \\
\hline & $\begin{array}{l}\text { CBS 248.67; CPC } 5117 \\
\text { (TYPE) }\end{array}$ & Althaea rosea & Malvaceae & Romania, Fundulea & O. Constantinescu & JX143530 & JX143284 & JX143038 & $J X 142792$ & JX142546 & MH496340 & - & MH496170 \\
\hline & СCTU 1194; IRAN $2674 C$ & Malva sylvestris & Malvaceae & $\begin{array}{l}\text { Iran, East } \\
\text { Azerbaijan, Kaleybar }\end{array}$ & M. Arzanlou & KJ886397 & KJ886236 & KJ885914 & KJ885753 & KJ886075 & MH496341 & MH511837 & MH496171 \\
\hline & CCTU 1071 & Malva sylvestris & Malvaceae & Iran, Guilan, Talesh & M. Bakhshi & KJ886395 & KJ886234 & KJ885912 & KJ885751 & KJ886073 & MH496342 & MH511838 & MH496172 \\
\hline \multirow[t]{8}{*}{ Cercospora apii } & $\begin{array}{l}\text { CBS 116455; CPC } 11556 \\
\text { (TYPE) }\end{array}$ & Apium graveolens & Apiaceae & Germany, Heilbron & K. Schrameyer & AY840519 & AY840486 & AY840450 & AY840417 & AY840384 & MH496343 & - & MH496173 \\
\hline & CBS 536.71; CPC 5087 & Apium graveolens & Apiaceae & Romania, Bucuresti & O. Constantinescu & AY752133 & AY752166 & AY752194 & AY752225 & AY752256 & MH496344 & MH511839 & MH496174 \\
\hline & ССTU 1069 & Cynanchum acutum & Apocynaceae & $\begin{array}{l}\text { Iran, Ardabil, } \\
\text { Moghan }\end{array}$ & M. Bakhshi & KJ886410 & KJ886249 & KJ885927 & KJ885766 & KJ886088 & MH496345 & MH511840 & MH496175 \\
\hline & $\begin{array}{l}\text { CCTU 1086; CBS 136037; } \\
\text { IRAN 2655C }\end{array}$ & Cynanchum acutum & Apocynaceae & $\begin{array}{l}\text { Iran, Ardabil, } \\
\text { Moghan }\end{array}$ & M. Bakhshi & KJ886411 & KJ886250 & KJ885928 & KJ885767 & KJ886089 & MH496346 & MH511841 & MH496176 \\
\hline & CCTU 1215 & Cynanchum acutum & Apocynaceae & $\begin{array}{l}\text { Iran, Ardabil, } \\
\text { Moghan }\end{array}$ & M. Bakhshi & KJ886412 & KJ886251 & KJ885929 & KJ885768 & KJ886090 & MH496347 & MH511842 & MH496177 \\
\hline & СCTU 1219; CBS 136155 & Cynanchum acutum & Apocynaceae & $\begin{array}{l}\text { Iran, Ardabil, } \\
\text { Moghan }\end{array}$ & M. Bakhshi & KJ886413 & KJ886252 & KJ885930 & KJ885769 & KJ886091 & MH496348 & MH511843 & MH496178 \\
\hline & CPC 5112 & Molucella laevis & Lamiaceae & $\begin{array}{l}\text { New zealand, } \\
\text { Auckland }\end{array}$ & C.F. Hill & DQ233321 & DQ233347 & DQ233373 & DQ233399 & DQ233425 & MH496349 & MH511844 & MH496179 \\
\hline & $\begin{array}{l}\text { CBS 110813; CPC 5110; } \\
01-3\end{array}$ & Molucella laevis & Lamiaceae & U.S.A., California & S.T. Koike & AY156918 & DQ233345 & DQ233371 & DQ233397 & DQ233423 & MH496350 & MH511845 & MH496180 \\
\hline Cercospora armoraciae & $\begin{array}{l}\text { CBS 250.67; CPC } 5088 \\
\text { (TYPE) }\end{array}$ & $\begin{array}{l}\text { Armoracia rusticana } \\
\text { (= A. lapathifolia) }\end{array}$ & Brassicaceae & Romania, Fundulea & O. Constantinescu & JX143545 & $J X 143299$ & JX143053 & $J \times 142807$ & $J X 142561$ & MH496351 & - & MH496181 \\
\hline \multirow[t]{9}{*}{ Cercospora beticola } & CPC 12028 & Beta vulgaris & Chenopodiaceae & Egypt & M. Hasem & DQ233336 & DQ233362 & DQ233388 & DQ233414 & DQ233437 & MH496352 & MH511846 & MH496182 \\
\hline & CPC 12029 & Beta vulgaris & Chenopodiaceae & Egypt & M. Hasem & DQ233337 & DQ233363 & DQ233389 & DQ233415 & DQ233438 & MH496353 & MH511847 & MH496183 \\
\hline & CCTU 1135 & Beta vulgaris & Chenopodiaceae & Iran, Guilan, Astara & M. Bakhshi & KJ886432 & KJ886271 & KJ885949 & KJ885788 & KJ886110 & MH496354 & MH511848 & MH496184 \\
\hline & $\begin{array}{l}\text { CBS 116456; CPC } 11557 \\
\text { (TYPE) }\end{array}$ & Beta vulgaris & Chenopodiaceae & Italy, Ravenna & V. Rossi & AY840527 & AY840494 & AY840458 & AY840425 & AY840392 & MH496355 & KT216555 & MH496185 \\
\hline & СCTU 1057; IRAN $2651 \mathrm{C}$ & Chenopodium sp. & Chenopodiaceae & $\begin{array}{l}\text { Iran, Ardabil, } \\
\text { Moghan }\end{array}$ & M. Bakhshi & KJ886424 & KJ886263 & KJ885941 & KJ885780 & KJ886102 & MH496356 & MH511849 & MH496186 \\
\hline & CCTU 1065 & Chenopodium sp. & Chenopodiaceae & $\begin{array}{l}\text { Iran, Ardabil, } \\
\text { Moghan }\end{array}$ & M. Bakhshi & KJ886425 & KJ886264 & KJ885942 & KJ885781 & KJ886103 & MH496357 & MH511850 & MH496187 \\
\hline & CCTU 1087 & Chenopodium sp. & Chenopodiaceae & $\begin{array}{l}\text { Iran, Ardabil, } \\
\text { Moghan }\end{array}$ & M. Bakhshi & KJ886427 & KJ886266 & KJ885944 & KJ885783 & KJ886105 & MH496358 & MH511851 & MH496188 \\
\hline & ССTU 1089; CРC 24911 & Plantago lanceolata & Plantaginaceae & $\begin{array}{l}\text { Iran, Ardabil, } \\
\text { Moghan }\end{array}$ & M. Bakhshi & KJ886429 & KJ886268 & KJ885946 & KJ885785 & KJ886107 & MH496359 & MH511852 & MH496189 \\
\hline & CCTU 1108 & Plantago lanceolata & Plantaginaceae & Iran, Zanjan, Tarom & M. Bakhshi & KJ886430 & KJ886269 & KJ885947 & KJ885786 & KJ886108 & MH496360 & MH511853 & MH496190 \\
\hline
\end{tabular}


Table 1. (Continued).

\begin{tabular}{|c|c|c|c|c|c|c|c|c|c|c|c|c|c|}
\hline \multirow[t]{2}{*}{ Species } & \multirow{2}{*}{$\begin{array}{l}\text { Culture accession } \\
\text { number }(\mathbf{s})^{1}\end{array}$} & \multirow[t]{2}{*}{ Host } & \multirow[t]{2}{*}{ Host Family } & \multirow[t]{2}{*}{ Origion } & \multirow[t]{2}{*}{ Collector } & \multicolumn{8}{|c|}{ GenBank accession numbers ${ }^{2}$} \\
\hline & & & & & & ITS & tef1 & actA & $c m d A$ & his3 & tub2 & rpb2 & gapdh \\
\hline & CCTU 1088; CBS 138582 & Sonchus asper & Asteraceae & $\begin{array}{l}\text { Iran, Ardabil, } \\
\text { Moghan }\end{array}$ & M. Bakhshi & KJ886428 & KJ886267 & KJ885945 & KJ885784 & KJ886106 & MH496361 & MH511854 & MH496191 \\
\hline \multirow[t]{9}{*}{$\begin{array}{l}\text { Cercospora } \\
\quad \text { bizzozeriana }\end{array}$} & CCTU 1013 & $?$ & $?$ & $\begin{array}{l}\text { Iran, East } \\
\text { Azerbaijan, Mianeh }\end{array}$ & M. Torbati & KJ886414 & KJ886253 & KJ885931 & KJ885770 & KJ886092 & MH496362 & MH511855 & MH496192 \\
\hline & CCTU 1022; CBS 136028 & $?$ & $?$ & $\begin{array}{l}\text { Iran, East } \\
\text { Azerbaijan, Mianeh }\end{array}$ & M. Torbati & KJ886415 & KJ886254 & KJ885932 & KJ885771 & KJ886093 & MH496363 & MH511856 & MH496193 \\
\hline & CCTU 1127; CBS 136133 & Capparis spinosa & Capparidaceae & $\begin{array}{l}\text { Iran, Khuzestan, } \\
\text { Ahvaz }\end{array}$ & E. Mohammadian & KJ886420 & KJ886259 & KJ885937 & KJ885776 & KJ886098 & MH496364 & MH511857 & MH496194 \\
\hline & CCTU 1117; CBS 136132 & Cardaria draba & Brassicaceae & $\begin{array}{l}\text { Iran, West } \\
\text { Azerbaijan, Khoy }\end{array}$ & M. Arzanlou & KJ886418 & KJ886257 & KJ885935 & KJ885774 & KJ886096 & MH496365 & MH511858 & MH496195 \\
\hline & CCTU 1234 & Cardaria draba & Brassicaceae & $\begin{array}{l}\text { Iran, West } \\
\text { Azerbaijan, Khoy }\end{array}$ & M. Arzanlou & KJ886419 & KJ886258 & KJ885936 & KJ885775 & KJ886097 & MH496366 & MH511859 & MH496196 \\
\hline & CCTU 1107 & $?$ & $?$ & Iran, Zanjan, Tarom & M. Bakhshi & KJ886417 & KJ886256 & KJ885934 & KJ885773 & KJ886095 & MH496367 & MH511860 & MH496197 \\
\hline & $\begin{array}{l}\text { CBS 258.67; CPC } 5061 \\
\text { (TYPE) }\end{array}$ & Cardaria draba & Brassicaceae & Romania, Fundulea & O. Constantinescu & JX143546 & JX143300 & $J X 143054$ & JX142808 & $J X 142562$ & MH496368 & - & MH496198 \\
\hline & $\begin{array}{l}\text { CBS 540.71; IMI 161110; } \\
\text { CPC } 5060\end{array}$ & Cardaria draba & Brassicaceae & Romania, Hagieni & O. Constantinescu & $J X 143548$ & $J X 143302$ & JX143056 & $J X 142810$ & JX142564 & MH496369 & - & MH496199 \\
\hline & CCTU 1040; CBS 136131 & Tanacetum balsamita & Asteraceae & Iran, Zanjan, Tarom & M. Bakhshi & KJ886416 & KJ886255 & KJ885933 & KJ885772 & KJ886094 & MH496370 & MH511861 & MH496200 \\
\hline \multirow[t]{3}{*}{ Cercospora chenopodii } & CCTU 1060; IRAN 2652C & Chenopodium album & Chenopodiaceae & $\begin{array}{l}\text { Iran, Guilan, } \\
\text { Bandar-e Anzali }\end{array}$ & M. Bakhshi & KJ886438 & KJ886277 & KJ885955 & KJ885794 & KJ886116 & MH496371 & MH511862 & MH496201 \\
\hline & CCTU 1163 & Chenopodium album & Chenopodiaceae & Iran, Guilan, Lahijan & M. Bakhshi & KJ886440 & KJ886279 & KJ885957 & KJ885796 & KJ886118 & MH496372 & MH511863 & MH496202 \\
\hline & CCTU 1033 & Chenopodium album & Chenopodiaceae & Iran, Guilan, Talesh & M. Bakhshi & KJ886437 & KJ886276 & KJ885954 & KJ885793 & KJ886115 & MH496373 & MH511864 & MH496203 \\
\hline \multirow[t]{2}{*}{$\begin{array}{l}\text { Cercospora } \\
\quad \text { convolvulicola }\end{array}$} & $\begin{array}{l}\text { CCTU 1083; CBS } 136126 \\
\text { (TYPE) }\end{array}$ & Convolvulus arvensis & Convolvulaceae & $\begin{array}{l}\text { Iran, Ardabil, } \\
\text { Moghan }\end{array}$ & M. Bakhshi & KJ886441 & KJ886280 & KJ885958 & KJ885797 & KJ886119 & MH496374 & MH511865 & MH496204 \\
\hline & CCTU 1083.2 & Convolvulus arvensis & Convolvulaceae & $\begin{array}{l}\text { Iran, Ardabil, } \\
\text { Moghan }\end{array}$ & M. Bakhshi & KJ886442 & KJ886281 & KJ885959 & KJ885798 & KJ886120 & MH496375 & MH511866 & MH496205 \\
\hline \multirow{3}{*}{$\begin{array}{l}\text { Cercospora conyzae- } \\
\quad \text { canadensis }\end{array}$} & CCTU 1008 & Conyza canadensis & Asteraceae & Iran, Guilan, Talesh & M. Bakhshi & KJ886443 & KJ886282 & KJ885960 & KJ885799 & KJ886121 & MH496376 & MH511867 & MH496206 \\
\hline & $\begin{array}{l}\text { CCTU 1119; CBS } 135978 \\
\text { (TYPE) }\end{array}$ & Conyza canadensis & Asteraceae & Iran, Guilan, Talesh & M. Bakhshi & KJ886445 & KJ886284 & KJ885962 & KJ885801 & KJ886123 & MH496377 & MH511868 & MH496207 \\
\hline & CCTU 1105; IRAN 2657C & Conyza canadensis & Asteraceae & Iran, Zanjan, Tarom & M. Bakhshi & KJ886444 & KJ886283 & KJ885961 & KJ885800 & KJ886122 & MH496378 & MH511869 & MH496208 \\
\hline \multirow[t]{7}{*}{ Cercospora cylindracea } & CCTU 1016 & Cichorium intybus & Asteraceae & $\begin{array}{l}\text { Iran, West } \\
\text { Azerbaijan, Khoy }\end{array}$ & M. Arzanlou & KJ886446 & KJ886285 & KJ885963 & KJ885802 & KJ886124 & MH496379 & MH511870 & MH496209 \\
\hline & CCTU 1114 & Cichorium intybus & Asteraceae & Iran, Zanjan, Tarom & M. Bakhshi & KJ886450 & KJ886289 & KJ885967 & KJ885806 & KJ886128 & MH496380 & MH511871 & MH496210 \\
\hline & $\begin{array}{l}\text { CCTU 1081; CBS 138580; } \\
\text { IRAN 2654C (TYPE) }\end{array}$ & Lactuca serriola & Asteraceae & $\begin{array}{l}\text { Iran, Ardabil, } \\
\text { Moghan }\end{array}$ & M. Bakhshi & KJ886449 & KJ886288 & KJ885966 & KJ885805 & KJ886127 & MH496381 & MH511872 & MH496211 \\
\hline & CCTU 1207 & Lactuca serriola & Asteraceae & $\begin{array}{l}\text { Iran, Ardabil, } \\
\text { Moghan }\end{array}$ & M. Bakhshi & KJ886453 & KJ886292 & KJ885970 & KJ885809 & KJ886131 & MH496382 & MH511873 & MH496212 \\
\hline & СCTU 1044; CBS 136021 & Lactuca serriola & Asteraceae & $\begin{array}{l}\text { Iran, West } \\
\text { Azerbaijan, Khoy }\end{array}$ & M. Arzanlou & KJ886447 & KJ886286 & KJ885964 & KJ885803 & KJ886125 & MH496383 & MH511874 & MH496213 \\
\hline & CCTU 1183 & Lactuca serriola & Asteraceae & $\begin{array}{l}\text { Iran, West } \\
\text { Azerbaijan, Khoy }\end{array}$ & M. Arzanlou & KJ886451 & KJ886290 & KJ885968 & KJ885807 & KJ886129 & MH496384 & MH511875 & MH496214 \\
\hline & CCTU 1189 & Lactuca serriola & Asteraceae & $\begin{array}{l}\text { Iran, West } \\
\text { Azerbaijan, Khoy }\end{array}$ & M. Arzanlou & KJ886452 & KJ886291 & KJ885969 & KJ885808 & KJ886130 & MH496385 & MH511876 & MH496215 \\
\hline
\end{tabular}




\begin{tabular}{|c|c|c|c|c|c|c|c|c|c|c|c|c|c|}
\hline \multirow[t]{2}{*}{ Species } & \multirow{2}{*}{$\begin{array}{l}\text { Culture accession } \\
\text { number }(\mathbf{s})^{1}\end{array}$} & \multirow[t]{2}{*}{ Host } & \multirow[t]{2}{*}{ Host Family } & \multirow[t]{2}{*}{ Origion } & \multirow[t]{2}{*}{ Collector } & \multicolumn{8}{|c|}{ GenBank accession numbers ${ }^{2}$} \\
\hline & & & & & & ITS & tef1 & actA & $c m d A$ & his3 & tub2 & $r p b 2$ & gapdh \\
\hline & CCTU 1049 & Lactuca serriola & Asteraceae & Iran, Zanjan, Tarom & M. Bakhshi & KJ886448 & KJ886287 & KJ885965 & KJ885804 & KJ886126 & MH496386 & MH511877 & MH496216 \\
\hline \multirow{12}{*}{$\begin{array}{l}\text { Cercospora cf. } \\
\quad \text { flagellaris clade } 1\end{array}$} & CPC 5441 & Amaranthus sp. & Amaranthaceae & Fiji & C.F. Hill & $J \times 143611$ & JX143370 & JX143124 & JX142878 & JX142632 & MH496387 & MH511878 & MH496217 \\
\hline & CCTU 1159; CBS 136148 & Arachis hypogaea & Fabaceae & Iran, Guilan, Lahijan & M. Bakhshi & KJ886493 & KJ886332 & KJ886010 & KJ885849 & KJ886171 & MH496388 & MH511879 & MH496218 \\
\hline & CCTU 1162; IRAN 2670C & Citrullus lanatus & Cucurbitaceae & Iran, Guilan, Lahijan & M. Bakhshi & KJ886496 & KJ886335 & KJ886013 & KJ885852 & KJ886174 & MH496389 & MH511880 & MH496219 \\
\hline & CBS 132653; CPC 10884 & $\begin{array}{l}\text { Dysphania } \\
\text { ambrosioides (三 } \\
\text { Chenopodium } \\
\text { ambrosioides) }\end{array}$ & Chenopodiaceae & South Korea, Jeju & H.D. Shin & JX143603 & JX143361 & $J X 143115$ & JX142869 & $J X 142623$ & MH496390 & MH511881 & MH496220 \\
\hline & CCTU 1007; CBS 136031 & Hydrangea sp. & Hydrangeaceae & Iran, Guilan, Talesh & M. Bakhshi & KJ886456 & KJ886295 & KJ885973 & KJ885812 & KJ886134 & MH496391 & MH511882 & MH496221 \\
\hline & CCTU 1027; CBS 136034 & Lepidium sativum & Brassicaceae & $\begin{array}{l}\text { Iran, Guilan, } \\
\text { Chamkhaleh }\end{array}$ & M. Bakhshi & KJ886459 & KJ886298 & KJ885976 & KJ885815 & KJ886137 & MH496392 & MH511883 & MH496222 \\
\hline & $\begin{array}{l}\text { CCTU 1128; CBS 136141; } \\
\text { IRAN 2661C }\end{array}$ & Phaseolus vulgaris & Fabaceae & Iran, Guilan, Astara & M. Bakhshi & KJ886476 & KJ886315 & KJ885993 & KJ885832 & KJ886154 & MH496393 & MH511884 & MH496223 \\
\hline & CCTU 1168; IRAN 2715C & Phaseolus vulgaris & Fabaceae & $\begin{array}{l}\text { Iran, Guilan, } \\
\text { Kiashahr }\end{array}$ & M. Bakhshi & KJ886499 & KJ886338 & KJ886016 & KJ885855 & KJ886177 & MH496394 & MH511885 & MH496224 \\
\hline & CPC 1051 & Populus deltoides & Salicaceae & South Africa & P.W. Crous & AY260069 & JX143367 & $J X 143121$ & JX142875 & JX142629 & MH496395 & MH511886 & MH496225 \\
\hline & CCTU 1171 & Raphanus sativus & Brassicaceae & $\begin{array}{l}\text { Iran, Guilan, } \\
\text { Kiashahr }\end{array}$ & M. Bakhshi & KJ886500 & KJ886339 & KJ886017 & KJ885856 & KJ886178 & MH496396 & MH511887 & MH496226 \\
\hline & CCTU 1120 & Raphanus sativus & Brassicaceae & Iran, Guilan, Talesh & M. Bakhshi & KJ886475 & KJ886314 & KJ885992 & KJ885831 & KJ886153 & MH496397 & MH511888 & MH496227 \\
\hline & $\begin{array}{l}\text { CCTU 1031; CBS 136036; } \\
\text { IRAN 2648C }\end{array}$ & Urtica dioica & Urticaceae & $\begin{array}{l}\text { Iran, Guilan, } \\
\text { Sowme'eh Sara }\end{array}$ & M. Bakhshi & KJ886461 & KJ886300 & KJ885978 & KJ885817 & KJ886139 & MH496398 & MH511889 & MH496228 \\
\hline \multirow[t]{10}{*}{$\begin{array}{l}\text { Cercospora cf. } \\
\text { flagellaris clade } 2\end{array}$} & CCTU 1204 & Abutilon theophrasti & Malvaceae & $\begin{array}{l}\text { Iran, Ardabil, } \\
\text { Moghan }\end{array}$ & M. Bakhshi & KJ886505 & KJ886344 & KJ886022 & KJ885861 & KJ886183 & MH496399 & MH511890 & MH496229 \\
\hline & CCTU 1198; CBS 136151 & Acer velutinum & Aceraceae & $\begin{array}{l}\text { Iran, Mazandaran, } \\
\text { Ramsar }\end{array}$ & M. Bakhshi & KJ886504 & KJ886343 & KJ886021 & KJ885860 & KJ886182 & MH496400 & MH511891 & MH496230 \\
\hline & CBS 132667; CPC 11643 & $\begin{array}{l}\text { Celosia argentea var. } \\
\text { cristata ( } \equiv \text { C. cristata) }\end{array}$ & Amaranthaceae & $\begin{array}{l}\text { South Korea, } \\
\text { Hoengseong }\end{array}$ & H.D. Shin & JX143604 & JX143362 & $J X 143116$ & JX142870 & $J X 142624$ & MH496401 & MH511892 & MH496231 \\
\hline & $\begin{array}{l}\text { CCTU 1115; CBS 136139; } \\
\text { IRAN 2659C }\end{array}$ & Cercis siliquastrum & Caesalpinaceae & Iran, Guilan, Astara & M. Bakhshi & KJ886473 & KJ886312 & KJ885990 & KJ885829 & KJ886151 & MH496402 & MH511893 & MH496232 \\
\hline & СCTU 1195 & Datura stramonium & Solanaceae & Iran, Guilan, Talesh & M. Bakhshi & KJ886503 & KJ886342 & KJ886020 & KJ885859 & KJ886181 & MH496403 & MH511894 & MH496233 \\
\hline & CCTU 1059; CBS 136136 & Ecballium elaterium & Cucurbitaceae & $\begin{array}{l}\text { Iran, Ardabil, } \\
\text { Moghan }\end{array}$ & M. Bakhshi & KJ886464 & KJ886303 & KJ885981 & KJ885820 & KJ886142 & MH496404 & MH511895 & MH496234 \\
\hline & CCTU 1216; IRAN 2717C & Ecballium elaterium & Cucurbitaceae & $\begin{array}{l}\text { Iran, Ardabil, } \\
\text { Moghan }\end{array}$ & M. Bakhshi & KJ886510 & KJ886349 & KJ886027 & KJ885866 & KJ886188 & MH496405 & MH511896 & MH496235 \\
\hline & $\begin{array}{l}\text { CCTU 1223; CBS 136154; } \\
\text { IRAN 2683C }\end{array}$ & Eclipta prostrata & Asteraceae & Iran, Guilan, Talesh & M. Bakhshi & KJ886512 & KJ886351 & KJ886029 & KJ885868 & KJ886190 & MH496406 & MH511897 & MH496236 \\
\hline & CCTU 1068 & Xanthium spinosum & Asteraceae & $\begin{array}{l}\text { Iran, Ardabil, } \\
\text { Moghan }\end{array}$ & M. Bakhshi & KJ886466 & KJ886305 & KJ885983 & KJ885822 & KJ886144 & MH496407 & MH511898 & MH496237 \\
\hline & CCTU 1085 & Xanthium strumarium & Asteraceae & $\begin{array}{l}\text { Iran, Ardabil, } \\
\text { Moghan }\end{array}$ & M. Bakhshi & KJ886471 & KJ886310 & KJ885988 & KJ885827 & KJ886149 & MH496408 & MH511899 & MH496238 \\
\hline
\end{tabular}


Table 1. (Continued).

\begin{tabular}{|c|c|c|c|c|c|c|c|c|c|c|c|c|c|}
\hline \multirow[t]{2}{*}{ Species } & \multirow{2}{*}{$\begin{array}{l}\text { Culture accession } \\
\text { number }(\mathbf{s})^{1}\end{array}$} & \multirow[t]{2}{*}{ Host } & \multirow[t]{2}{*}{ Host Family } & \multirow[t]{2}{*}{ Origion } & \multirow[t]{2}{*}{ Collector } & \multicolumn{8}{|c|}{ GenBank accession numbers ${ }^{2}$} \\
\hline & & & & & & ITS & tef1 & $\operatorname{act} A$ & $c m d A$ & his3 & tub2 & rpb2 & gapdh \\
\hline \multirow{27}{*}{$\begin{array}{l}\text { Cercospora cf. } \\
\quad \text { flagellaris clade } 3\end{array}$} & ССTU 1172 & Oenothera biennis & Onagraceae & Iran, Guilan, Talesh & M. Bakhshi & KJ886501 & KJ886340 & KJ886018 & KJ885857 & KJ886179 & MH496409 & MH511900 & MH496239 \\
\hline & СCTU 1154; CBS 136147 & Abutilon theophrasti & Malvaceae & Iran, Guilan, Rasht & M. Bakhshi & KJ886489 & KJ886328 & KJ886006 & KJ885845 & KJ886167 & MH496410 & MH511901 & MH496240 \\
\hline & CCTU 1072; IRAN $2653 \mathrm{C}$ & Amaranthus blitoides & Amaranthaceae & $\begin{array}{l}\text { Iran, Ardabil, } \\
\text { Moghan }\end{array}$ & M. Bakhshi & KJ886468 & KJ886307 & KJ885985 & KJ885824 & KJ886146 & MH496411 & MH511902 & MH496241 \\
\hline & CCTU 1064 & $\begin{array}{l}\text { Amaranthus } \\
\text { retroflexus }\end{array}$ & Amaranthaceae & $\begin{array}{l}\text { Iran, Ardabil, } \\
\text { Moghan }\end{array}$ & M. Bakhshi & KJ886465 & KJ886304 & KJ885982 & KJ885821 & KJ886143 & MH496412 & MH511903 & MH496242 \\
\hline & CCTU 1021; CBS 136033 & $\begin{array}{l}\text { Amaranthus } \\
\text { retroflexus }\end{array}$ & Amaranthaceae & Iran, Guilan, Fuman & M. Bakhshi & KJ886458 & KJ886297 & KJ885975 & KJ885814 & KJ886136 & MH496413 & MH511904 & MH496243 \\
\hline & CCTU 1084; CBS 136156 & Amaranthus sp. & Amaranthaceae & $\begin{array}{l}\text { Iran, Ardabil, } \\
\text { Moghan }\end{array}$ & M. Bakhshi & KJ886470 & KJ886309 & KJ885987 & KJ885826 & KJ886148 & MH496414 & MH511905 & MH496244 \\
\hline & CCTU 1167; CBS 136150 & Anubias sp. & Araceae & $\begin{array}{l}\text { Iran, Guilan, } \\
\text { Kiashahr }\end{array}$ & M. Bakhshi & KJ886498 & KJ886337 & KJ886015 & KJ885854 & KJ886176 & MH496415 & MH511906 & MH496245 \\
\hline & CBS 143.51; CPC 5055 & Bromus sp. & Poaceae & - & M.D. Whitehead & JX143607 & JX143365 & JX143119 & JX142873 & $J X 142627$ & MH496416 & MH511907 & MH496246 \\
\hline & ССTU 1150 & Buxus microphylla & Buxaceae & Iran, Guilan, Fuman & M. Bakhshi & KJ886488 & KJ886327 & KJ886005 & KJ885844 & KJ886166 & MH496417 & MH511908 & MH496247 \\
\hline & $\begin{array}{l}\text { CCTU 1140; CBS 136143; } \\
\text { IRAN 2666C }\end{array}$ & Calendula officinalis & Asteraceae & Iran, Guilan, Astara & M. Bakhshi & KJ886481 & KJ886320 & KJ885998 & KJ885837 & KJ886159 & MH496418 & MH511909 & MH496248 \\
\hline & $\begin{array}{l}\text { CBS } 115482 ; \text { A207 Bs+; } \\
\text { CPC } 4410\end{array}$ & Citrus sp. & Rutaceae & $\begin{array}{l}\text { South Africa, } \\
\text { Messina }\end{array}$ & M.C. Pretorius & AY260070 & DQ835095 & DQ835114 & DQ835141 & DQ835168 & MH496419 & MH511910 & MH496249 \\
\hline & $\begin{array}{l}\text { CCTU 1029; CBS 136035; } \\
\text { IRAN 2647C }\end{array}$ & Cucurbita maxima & Cucurbitaceae & Iran, Guilan, Rudsar & r M. Bakhshi & KJ886460 & KJ886299 & KJ885977 & KJ885816 & KJ886138 & MH496420 & MH511911 & MH496250 \\
\hline & ССTU 1136 & Cucurbita pepo & Cucurbitaceae & Iran, Guilan, Astara & M. Bakhshi & KJ886478 & KJ886317 & KJ885995 & KJ885834 & KJ886156 & MH496421 & MH511912 & MH496251 \\
\hline & CCTU 1143; CBS 136145 & Datura stramonium & Solanaceae & Iran, Guilan, Talesh & M. Bakhshi & KJ886484 & KJ886323 & KJ886001 & KJ885840 & KJ886162 & MH496422 & MH511913 & MH496252 \\
\hline & СCTU 1209; CBS 136152 & Glycine max & Fabaceae & $\begin{array}{l}\text { Iran, Ardabil, } \\
\text { Moghan }\end{array}$ & M. Bakhshi & KJ886506 & KJ886345 & KJ886023 & KJ885862 & KJ886184 & MH496423 & MH511914 & MH496253 \\
\hline & CCTU 1210; IRAN 2679C & Glycine max & Fabaceae & $\begin{array}{l}\text { Iran, Ardabil, } \\
\text { Moghan }\end{array}$ & M. Bakhshi & KJ886507 & KJ886346 & KJ886024 & KJ885863 & KJ886185 & MH496424 & MH511915 & MH496254 \\
\hline & ССТU 1211 & Glycine max & Fabaceae & $\begin{array}{l}\text { Iran, Ardabil, } \\
\text { Moghan }\end{array}$ & M. Bakhshi & KJ886508 & KJ886347 & KJ886025 & KJ885864 & KJ886186 & MH496425 & MH511916 & MH496255 \\
\hline & CCTU 1218; IRAN 2682C & Hibiscus trionum & Malvaceae & $\begin{array}{l}\text { Iran, Ardabil, } \\
\text { Moghan }\end{array}$ & M. Bakhshi & KJ886511 & KJ886350 & KJ886028 & KJ885867 & KJ886189 & MH496426 & MH511917 & MH496256 \\
\hline & CCTU 1006; CBS 136030 & Impatiens balsamina & Balsaminaceae & Iran, Guilan, Talesh & M. Bakhshi & KJ886455 & KJ886294 & KJ885972 & KJ885811 & KJ886133 & MH496427 & MH511918 & MH496257 \\
\hline & СCTU 1130; CBS 136142 & Olea europaea & Oleaceae & Iran, Zanjan, Tarom & M. Torbati & KJ886477 & KJ886316 & KJ885994 & KJ885833 & KJ886155 & MH496428 & MH511919 & MH496258 \\
\hline & CCTU 1010; CBS 136032 & Pelargonium hortorum & nGeraniaceae & Iran, Guilan, Talesh & M. Bakhshi & KJ886457 & KJ886296 & KJ885974 & KJ885813 & KJ886135 & MH496429 & MH511920 & MH496259 \\
\hline & CCTU 1138; IRAN 2664C & Phaseolus vulgaris & Fabaceae & Iran, Guilan, Astara & M. Bakhshi & KJ886479 & KJ886318 & KJ885996 & KJ885835 & KJ886157 & MH496430 & MH511921 & MH496260 \\
\hline & CCTU 1139; IRAN 2665C & Phaseolus vulgaris & Fabaceae & Iran, Guilan, Astara & M. Bakhshi & KJ886480 & KJ886319 & KJ885997 & KJ885836 & KJ886158 & MH496431 & MH511922 & MH496261 \\
\hline & CCTU 1155.11 & Phaseolus vulgaris & Fabaceae & Iran, Guilan, Fuman & M. Bakhshi & KJ886490 & KJ886329 & KJ886007 & KJ885846 & KJ886168 & MH496432 & MH511923 & MH496262 \\
\hline & ССTU 1161; IRAN $2669 \mathrm{C}$ & Phaseolus vulgaris & Fabaceae & Iran, Guilan, Lahijan & M. Bakhshi & KJ886495 & KJ886334 & KJ886012 & KJ885851 & KJ886173 & MH496433 & MH511924 & MH496263 \\
\hline & CCTU 1175; IRAN $2673 \mathrm{C}$ & Phaseolus vulgaris & Fabaceae & $\begin{array}{l}\text { Iran, Guilan, } \\
\text { Sowme'eh Sara }\end{array}$ & M. Bakhshi & KJ886502 & KJ886341 & KJ886019 & KJ885858 & KJ886180 & MH496434 & MH511925 & MH496264 \\
\hline & CCTU 1142; IRAN 2667C & Phaseolus vulgaris & Fabaceae & Iran, Guilan, Talesh & M. Bakhshi & KJ886483 & KJ886322 & KJ886000 & KJ885839 & KJ886161 & MH496435 & MH511926 & MH496265 \\
\hline
\end{tabular}




\begin{tabular}{|c|c|c|c|c|c|c|c|c|c|c|c|c|c|}
\hline \multirow[t]{2}{*}{ Species } & \multirow{2}{*}{$\begin{array}{l}\text { Culture accession } \\
\text { number }(\mathbf{s})^{1}\end{array}$} & \multirow[t]{2}{*}{ Host } & \multirow[t]{2}{*}{ Host Family } & \multirow[t]{2}{*}{ Origion } & \multirow[t]{2}{*}{ Collector } & \multicolumn{8}{|c|}{ GenBank accession numbers ${ }^{2}$} \\
\hline & & & & & & ITS & tef1 & actA & $c m d A$ & his3 & tub2 & $r p b 2$ & gapdh \\
\hline & $\begin{array}{l}\text { CCTU 1118; CBS 136140; } \\
\text { IRAN 2660C }\end{array}$ & Populus deltoides & Salicaceae & Iran, Guilan, Astara & M. Bakhshi & KJ886474 & KJ886313 & KJ885991 & KJ885830 & KJ886152 & MH496436 & MH511927 & MH496266 \\
\hline & CCTU 1075 & Raphanus sativus & Brassicaceae & $\begin{array}{l}\text { Iran, Guilan, } \\
\text { Sowme'eh Sara }\end{array}$ & M. Bakhshi & KJ886469 & KJ886308 & KJ885986 & KJ885825 & KJ886147 & MH496437 & MH511928 & MH496267 \\
\hline & $\begin{array}{l}\text { CCTU 1212; CBS 136153; } \\
\text { IRAN 2680C }\end{array}$ & ; Silybum marianum & Asteraceae & $\begin{array}{l}\text { Iran, Ardabil, } \\
\text { Moghan }\end{array}$ & M. Bakhshi & KJ886509 & KJ886348 & KJ886026 & KJ885865 & KJ886187 & MH496438 & MH511929 & MH496268 \\
\hline & CCTU 1141; CBS 136144 & Tagetes patula & Asteraceae & Iran, Guilan, Rudsar & ir M. Bakhshi & KJ886482 & KJ886321 & KJ885999 & KJ885838 & KJ886160 & MH496439 & MH511930 & MH496269 \\
\hline & CCTU 1147 & Urtica dioica & Urticaceae & Iran, Guilan, Masal & M. Bakhshi & KJ886486 & KJ886325 & KJ886003 & KJ885842 & KJ886164 & MH496440 & MH511931 & MH496270 \\
\hline & CCTU 1160; CBS 136149 & Vicia faba & Fabaceae & Iran, Guilan, Astara & M. Bakhshi & KJ886494 & KJ886333 & KJ886011 & KJ885850 & KJ886172 & MH496441 & MH511932 & MH496271 \\
\hline & CCTU 1158; IRAN 2668C & Xanthium strumarium & Asteraceae & $\begin{array}{l}\text { Iran, Guilan, } \\
\text { Langarud }\end{array}$ & M. Bakhshi & KJ886492 & KJ886331 & KJ886009 & KJ885848 & KJ886170 & MH496442 & MH511933 & MH496272 \\
\hline & СCTU 1156 & Xanthium strumarium & Asteraceae & Iran, Guilan, Rasht & M. Bakhshi & KJ886491 & KJ886330 & KJ886008 & KJ885847 & KJ886169 & MH496443 & MH511934 & MH496273 \\
\hline & CCTU 1005; IRAN 2644C & Xanthium strumarium & Asteraceae & Iran, Guilan, Talesh & M. Bakhshi & KJ886454 & KJ886293 & KJ885971 & KJ885810 & KJ886132 & MH496444 & MH511935 & MH496274 \\
\hline & CCTU 1048; CBS 136029 & Xanthium strumarium & Asteraceae & Iran, Zanjan, Tarom & M. Bakhshi & KJ886462 & KJ886301 & KJ885979 & KJ885818 & KJ886140 & MH496445 & MH511936 & MH496275 \\
\hline \multirow[t]{6}{*}{ Cercospora gamsiana } & $\begin{array}{l}\text { CBS 144962; CCTU 1074; } \\
\text { CPC } 24909 \text { (TYPE) }\end{array}$ & ; Malva neglecta & Malvaceae & $\begin{array}{l}\text { Iran, Ardabil, } \\
\text { Moghan }\end{array}$ & M. Bakhshi & KJ886426 & KJ886265 & KJ885943 & KJ885782 & KJ886104 & MH496446 & MH511937 & MH496276 \\
\hline & CCTU 1035 & Malva sylvestris & Malvaceae & Iran, Zanjan, Tarom & M. Bakhshi & KJ886423 & KJ886262 & KJ885940 & KJ885779 & KJ886101 & MH496447 & MH511938 & MH496277 \\
\hline & ССTU 1109 & Malva sylvestris & Malvaceae & Iran, Zanjan, Tarom & M. Bakhshi & KJ886431 & KJ886270 & KJ885948 & KJ885787 & KJ886109 & MH496448 & MH511939 & MH496278 \\
\hline & $\begin{array}{l}\text { CCTU 1199; CBS 136128; } \\
\text { IRAN 2675C }\end{array}$ & Rumex crispus & Polygonaceae & $\begin{array}{l}\text { Iran, Mazandaran, } \\
\text { Ramsar }\end{array}$ & M. Bakhshi & KJ886433 & KJ886272 & KJ885950 & KJ885789 & KJ886111 & MH496449 & MH511940 & MH496279 \\
\hline & $\begin{array}{l}\text { CCTU 1205; CBS 136127; } \\
\text { IRAN 2677C }\end{array}$ & ; Sesamum indicum & Pedaliaceae & $\begin{array}{l}\text { Iran, Ardabil, } \\
\text { Moghan }\end{array}$ & M. Bakhshi & KJ886435 & KJ886274 & KJ885952 & KJ885791 & KJ886113 & MH496450 & MH511941 & MH496280 \\
\hline & CCTU 1208; IRAN $2678 C$ & Sonchus sp. & Asteraceae & $\begin{array}{l}\text { Iran, Ardabil, } \\
\text { Moghan }\end{array}$ & M. Bakhshi & KJ886436 & KJ886275 & KJ885953 & KJ885792 & KJ886114 & MH496451 & MH511942 & MH496281 \\
\hline \multirow[t]{2}{*}{ Cercospora cf. gossypi } & ii CCTU 1070; CBS 136137 & $\begin{array}{l}\text { Gossypium } \\
\text { herbaceum }\end{array}$ & Malvaceae & $\begin{array}{l}\text { Iran, Ardabil, } \\
\text { Moghan }\end{array}$ & M. Bakhshi & KJ886467 & KJ886306 & KJ885984 & KJ885823 & KJ886145 & MH496452 & MH511943 & MH496282 \\
\hline & CCTU 1055; IRAN $2650 \mathrm{C}$ & Hibiscus trionum & Malvaceae & $\begin{array}{l}\text { Iran, Ardabil, } \\
\text { Moghan }\end{array}$ & M. Bakhshi & KJ886463 & KJ886302 & KJ885980 & KJ885819 & KJ886141 & MH496453 & MH511944 & MH496283 \\
\hline \multirow[t]{2}{*}{ Cercospora iranica } & CCTU 1196; CBS 136123 & Hydrangea sp. & Hydrangeaceae & $\begin{array}{l}\text { Iran, Mazandaran, } \\
\text { Ramsar }\end{array}$ & M. Bakhshi & KJ886515 & KJ886354 & KJ886032 & KJ885871 & KJ886193 & MH496454 & MH511945 & MH496284 \\
\hline & $\begin{array}{l}\text { CCTU 1137; CBS } 136124 \\
\text { (TYPE) }\end{array}$ & Vicia faba & Fabaceae & Iran, Guilan, Astara & M. Bakhshi & KJ886513 & KJ886352 & KJ886030 & KJ885869 & KJ886191 & MH496455 & MH511946 & MH496285 \\
\hline \multirow[t]{5}{*}{ Cercospora plantaginis } & CCTU 1082; CBS 138728 & Plantago lanceolata & Plantaginaceae & $\begin{array}{l}\text { Iran, Ardabil, } \\
\text { Moghan }\end{array}$ & M. Bakhshi & KJ886402 & KJ886241 & KJ885919 & KJ885758 & KJ886080 & MH496456 & MH511947 & MH496286 \\
\hline & ССTU 1095 & Plantago lanceolata & Plantaginaceae & $\begin{array}{l}\text { Iran, East } \\
\text { Azerbaijan, Horand }\end{array}$ & M. Bakhshi & KJ886403 & KJ886242 & KJ885920 & KJ885759 & KJ886081 & MH496457 & MH511948 & MH496287 \\
\hline & CCTU 1041; CPC 24910 & Plantago lanceolata & Plantaginaceae & $\begin{array}{l}\text { Iran, Guilan, } \\
\text { Chaboksar }\end{array}$ & M. Bakhshi & KJ886400 & KJ886239 & KJ885917 & KJ885756 & KJ886078 & MH496458 & MH511949 & MH496288 \\
\hline & CCTU 1179; IRAN $2716 \mathrm{C}$ & Plantago lanceolata & Plantaginaceae & $\begin{array}{l}\text { Iran, West } \\
\text { Azerbaijan, Khoy }\end{array}$ & M. Arzanlou & KJ886404 & KJ886243 & KJ885921 & KJ885760 & KJ886082 & MH496459 & MH511950 & MH496289 \\
\hline & CCTU 1047 & Plantago lanceolata & Plantaginaceae & Iran, Zanjan, Tarom & M. Bakhshi & KJ886401 & KJ886240 & KJ885918 & KJ885757 & KJ886079 & MH496460 & MH511951 & MH496290 \\
\hline
\end{tabular}


Table 1. (Continued).

\begin{tabular}{|c|c|c|c|c|c|c|c|c|c|c|c|c|c|}
\hline \multirow[t]{2}{*}{ Species } & \multirow{2}{*}{$\begin{array}{l}\text { Culture accession } \\
\text { number }(\mathbf{s})^{1}\end{array}$} & \multirow[t]{2}{*}{ Host } & \multirow[t]{2}{*}{ Host Family } & \multirow[t]{2}{*}{ Origion } & \multirow[t]{2}{*}{ Collector } & \multicolumn{8}{|c|}{ GenBank accession numbers ${ }^{2}$} \\
\hline & & & & & & ITS & tef1 & $\operatorname{act} A$ & $c m d A$ & his3 & tub2 & $r p b 2$ & gapdh \\
\hline & $\begin{array}{l}\text { CBS 252.67; CPC } 5084 \\
\text { (TYPE) }\end{array}$ & Plantago lanceolata & Plantaginaceae & Romania, Domnesti & O. Constantinescu & DQ233318 & DQ233342 & DQ233368 & DQ233394 & DQ233420 & MH496461 & - & MH496291 \\
\hline \multirow[t]{3}{*}{$\begin{array}{l}\text { Cercospora } \\
\quad \text { pseudochenopodii }\end{array}$} & ССTU 1176 & Chenopodium album & Chenopodiaceae & $\begin{array}{l}\text { Iran, West } \\
\text { Azerbaijan, Khoy }\end{array}$ & M. Arzanlou & KJ886518 & KJ886357 & KJ886035 & KJ885874 & KJ886196 & MH496462 & MH511952 & MH496292 \\
\hline & CCTU 1045 & Chenopodium sp. & Chenopodiaceae & $\begin{array}{l}\text { Iran, West } \\
\text { Azerbaijan, Khoy }\end{array}$ & M. Arzanlou & KJ886517 & KJ886356 & KJ886034 & KJ885873 & KJ886195 & MH496463 & MH511953 & MH496293 \\
\hline & $\begin{array}{l}\text { CCTU 1038; CBS 136022; } \\
\text { IRAN 2649C (TYPE) }\end{array}$ & Chenopodium sp. & Chenopodiaceae & Iran, Zanjan, Tarom & M. Bakhshi & KJ886516 & KJ886355 & KJ886033 & KJ885872 & KJ886194 & MH496464 & MH511954 & MH496294 \\
\hline $\begin{array}{l}\text { Cercospora cf. } \\
\text { richardiicola }\end{array}$ & СCTU 1004 & Bidens tripartita & Asteraceae & Iran, Guilan, Talesh & M. Bakhshi & KJ886519 & KJ886358 & KJ886036 & KJ885875 & KJ886197 & MH496465 & MH511955 & MH496295 \\
\hline \multirow[t]{3}{*}{ Cercospora rumicis } & CCTU 1123 & Rumex crispus & Polygonaceae & Iran, Guilan, Talesh & M. Bakhshi & KJ886521 & KJ886360 & KJ886038 & KJ885877 & KJ886199 & MH496466 & MH511956 & MH496296 \\
\hline & CCTU 1129; IRAN 2662C & Rumex crispus & Polygonaceae & Iran, Guilan, Talesh & M. Bakhshi & KJ886522 & KJ886361 & KJ886039 & KJ885878 & KJ886200 & MH496467 & MH511957 & MH496297 \\
\hline & CCTU 1121 & Urtica dioica & Urticaceae & Iran, Guilan, Talesh & M. Bakhshi & KJ886520 & KJ886359 & KJ886037 & KJ885876 & KJ886198 & MH496468 & MH511958 & MH496298 \\
\hline \multirow[t]{2}{*}{ Cercospora solani } & CCTU 1043; CBS 136038 & Solanum nigrum & Solanaceae & $\begin{array}{l}\text { Iran, West } \\
\text { Azerbaijan, Khoy }\end{array}$ & M. Arzanlou & KJ886523 & KJ886362 & KJ886040 & KJ885879 & KJ886201 & MH496469 & MH511959 & MH496299 \\
\hline & CCTU 1050 & Solanum nigrum & Solanaceae & $\begin{array}{l}\text { Iran, West } \\
\text { Azerbaijan, Khoy }\end{array}$ & M. Arzanlou & KJ886524 & KJ886363 & KJ886041 & KJ885880 & KJ886202 & MH496470 & MH511960 & MH496300 \\
\hline Cercospora sorghicola & $\begin{array}{l}\text { CCTU 1173; CBS 136448; } \\
\text { IRAN 2672C (TYPE) }\end{array}$ & Sorghum halepense & Poaceae & $\begin{array}{l}\text { Iran, Guilan, } \\
\text { Kiashahr }\end{array}$ & M. Bakhshi & KJ886525 & KJ886364 & KJ886042 & KJ885881 & KJ886203 & MH496471 & MH511961 & MH496301 \\
\hline \multirow{3}{*}{$\begin{array}{l}\text { Cercospora sp. G } \\
\quad \text { clade } 1\end{array}$} & CCTU 1197 & Bidens tripartita & Asteraceae & Iran, Guilan, Talesh & M. Bakhshi & KJ886540 & KJ886379 & KJ886057 & KJ885896 & KJ886218 & MH496472 & MH511962 & MH496302 \\
\hline & $\begin{array}{l}\text { CCTU 1015; CBS 136024; } \\
\text { IRAN 2645C }\end{array}$ & Plantago major & Plantaginaceae & Iran, Guilan, Talesh & M. Bakhshi & KJ886528 & KJ886367 & KJ886045 & KJ885884 & KJ886206 & MH496473 & MH511963 & MH496303 \\
\hline & CPC 5438 & Salvia viscosa & Lamiaceae & $\begin{array}{l}\text { New Zealand, } \\
\text { Manurewa }\end{array}$ & C.F. Hill & JX143682 & JX143442 & JX143196 & JX142950 & $J X 142704$ & MH496474 & _- & MH496304 \\
\hline \multirow[t]{10}{*}{$\begin{array}{l}\text { Cercospora sp. G } \\
\quad \text { clade } 2\end{array}$} & CCTU 1058 & $\begin{array}{l}\text { Helminthotheca } \\
\text { echioides }\end{array}$ & Asteraceae & $\begin{array}{l}\text { Iran, Ardabil, } \\
\text { Moghan }\end{array}$ & M. Bakhshi & KJ886534 & KJ886373 & KJ886051 & KJ885890 & KJ886212 & MH496475 & MH511964 & MH496305 \\
\hline & CCTU 1090 & Abutilon theophrasti & Malvaceae & $\begin{array}{l}\text { Iran, Ardabil, } \\
\text { Moghan }\end{array}$ & M. Bakhshi & KJ886536 & KJ886375 & KJ886053 & KJ885892 & KJ886214 & MH496476 & MH511965 & MH496306 \\
\hline & СCTU 1079; CBS 136025 & $\begin{array}{l}\text { Amaranthus } \\
\text { retroflexus }\end{array}$ & Amaranthaceae & $\begin{array}{l}\text { Iran, Ardabil, } \\
\text { Moghan }\end{array}$ & M. Bakhshi & KJ886535 & KJ886374 & KJ886052 & KJ885891 & KJ886213 & MH496477 & MH511966 & MH496307 \\
\hline & CCTU 1054 & Amaranthus sp. & Amaranthaceae & $\begin{array}{l}\text { Iran, Ardabil, } \\
\text { Moghan }\end{array}$ & M. Bakhshi & KJ886533 & KJ886372 & KJ886050 & KJ885889 & KJ886211 & MH496478 & MH511967 & MH496308 \\
\hline & CCTU 1122 & Amaranthus sp. & Amaranthaceae & Iran, Guilan, Talesh & M. Bakhshi & KJ886538 & KJ886377 & KJ886055 & KJ885894 & KJ886216 & MH496479 & MH511968 & MH496309 \\
\hline & CBS 115518; CPC 5360 & Bidens frondosa & Asteraceae & $\begin{array}{l}\text { New Zealand, } \\
\text { Kopuku }\end{array}$ & C.F. Hill & JX143681 & JX143441 & JX143195 & JX142949 & JX142703 & MH496480 & - & MH496310 \\
\hline & CCTU 1030; CBS 136026 & Bidens tripartita & Asteraceae & Iran, Guilan, Talesh & M. Bakhshi & KJ886530 & KJ886369 & KJ886047 & KJ885886 & KJ886208 & MH496481 & MH511969 & MH496311 \\
\hline & CCTU 1002 & Celosia cristata & Amaranthaceae & Iran, Guilan, Talesh & M. Bakhshi & KJ886527 & KJ886366 & KJ886044 & KJ885883 & KJ886205 & MH496482 & MH511970 & MH496312 \\
\hline & ССTU 1053; CBS 136027 & Cichorium intybus & Asteraceae & $\begin{array}{l}\text { Iran, Guilan, } \\
\text { Sowme'eh Sara }\end{array}$ & M. Bakhshi & KJ886532 & KJ886371 & KJ886049 & KJ885888 & KJ886210 & MH496483 & MH511971 & MH496313 \\
\hline & $\begin{array}{l}\text { CCTU 1144; CBS } \\
136130\end{array}$ & Cucurbita maxima & Cucurbitaceae & $\begin{array}{l}\text { Iran, Guilan, } \\
\text { Masal }\end{array}$ & M. Bakhshi & KJ886539 & 9 KJ886378 & KJ886056 & 6KJ885895 & 5 KJ886217 & MH496484 & MH511972 & MH496314 \\
\hline
\end{tabular}




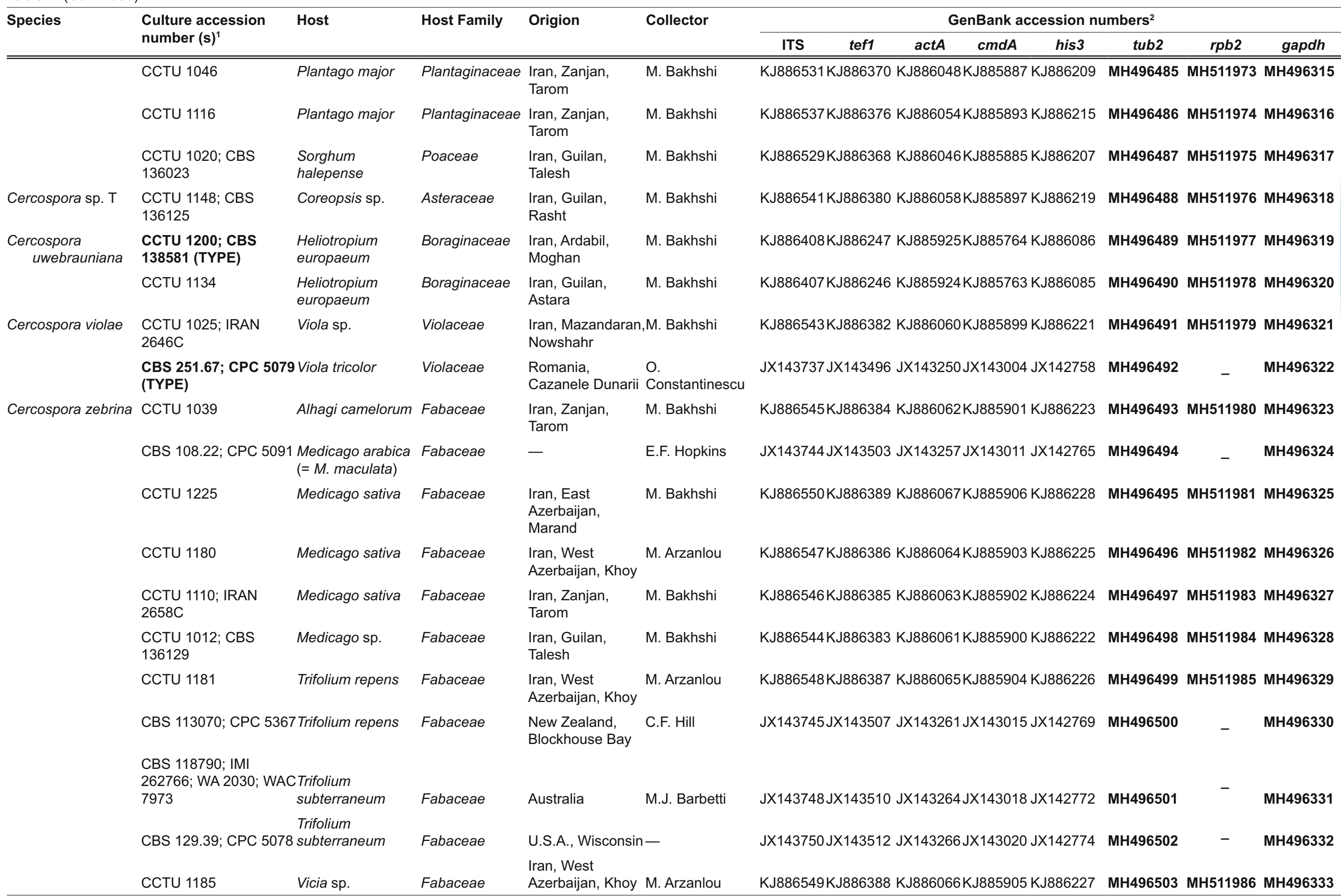


Table 1. (Continued).

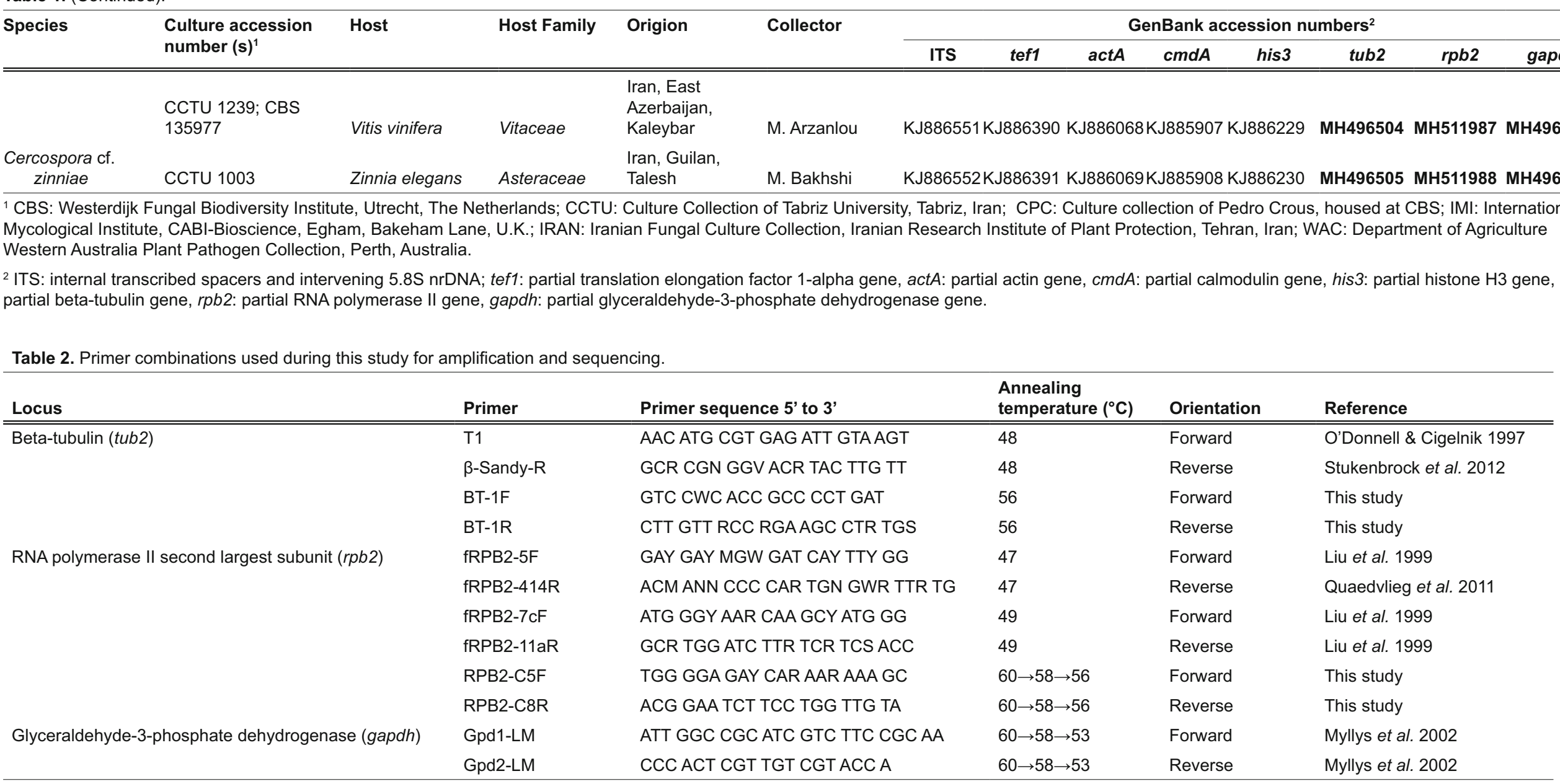

Table 3. Phylogenetic data and the substitution models used in the phylogenetic analysis, per locus. Abbreviations of loci follow Table 1.

\begin{tabular}{|c|c|c|c|c|c|c|c|c|}
\hline Locus & ITS & tef1 & act $A$ & $c m d A$ & his3 & tub2 & $r p b 2$ & gapdh \\
\hline Number of characters & 470 & 291 & 187 & 248 & 358 & 415 & 1229 & 869 \\
\hline Unique site patterns & 16 & 75 & 48 & 66 & 63 & 105 & 259 & 231 \\
\hline Substitution model used & SYM-gamma & K80-gamma & K80-gamma & K80-gamma & HKY-gamma & GTR-gamma & GTR-gamma & GTR-I-gamma \\
\hline Number of generations $(n)$ & \multicolumn{8}{|c|}{2405000} \\
\hline Total number of trees $(n)$ & \multicolumn{8}{|c|}{4812} \\
\hline Sampled trees $(n)$ & \multicolumn{8}{|c|}{3610} \\
\hline
\end{tabular}


polymerase (Promega). The tub2 PCR mixture contained 5-10 ng genomic DNA, $1 \times$ PCR buffer, $2 \mathrm{mM} \mathrm{MgCl}_{2}, 40 \mu \mathrm{M}$ of each dNTP, $0 \mu \mathrm{L} / 0.5 \mu \mathrm{L}$ BSA, $0.25 \mu \mathrm{M}$ of each primer and 0.5 units GoTaq ${ }^{\circledR}$ Flexi DNA polymerase using respectively the BT-1F/BT-1R (this study) or T1 (O'Donnell \& Cigelnik 1997)/ $\beta-S a n d y-R$ (Stukenbrock et al. 2012) primer sets. The rpb2 gene was amplified in three parts with three primer sets. Part three was only amplified in some selected species in order to design a new reverse primer for amplification of part two. The rpb2 PCR mixtures using the fRPB2-5F (Liu et al. 1999)/fRPB2-414R (Quaedvlieg et al. 2011) primer set consisted of 5-10 ng genomic DNA, $1 \times$ PCR buffer, $2 \mathrm{mM}$ $\mathrm{MgCl}_{2}, 40 \mu \mathrm{M}$ of each dNTP, $0.5 \mu \mathrm{L}$ BSA, $0.2 \mu \mathrm{M}$ of each primer and 0.5 units GoTaq ${ }^{\circledR}$ Flexi DNA polymerase. The PCR mixtures using RPB2-C5F/RPB2-C8R (this study) and fRPB2-7cF/fRPB2-11aR primer sets (Liu et al. 1999) were the same as gapdh.

To obtain the partial tub2 and rpb2 (using the fRPB25F/fRPB2-414R and fRPB2-7cF/fRPB2-11aR primer sets) sequences, $\mathrm{PCR}$ amplification conditions were set as follows: an initial denaturation temperature of $94^{\circ} \mathrm{C}$ for $3 \mathrm{~min}$, followed by 40 (tub2) or 45 ( $r p b 2$ ) cycles of denaturation temperature of $94{ }^{\circ} \mathrm{C}$ for $30 \mathrm{~s}$, primer annealing at the temperature stipulated in Table 2 for $30 \mathrm{~s}$, primer extension at $72{ }^{\circ} \mathrm{C}$ for $45 \mathrm{~s}$ and a final extension step at $72{ }^{\circ} \mathrm{C}$ for $5 \mathrm{~min}$.

A touchdown PCR protocol was used to amplify the partial gapdh (using the Gpd1-LM/Gpd2-LM primer set (Myllys et al. 2002)) and rpb2 (using the RPB2-C5F/RPB2-C8R primer set) sequences: initial denaturation $\left(94^{\circ} \mathrm{C}, 5 \mathrm{~min}\right)$, five amplification cycles $\left(94^{\circ} \mathrm{C}, 45 \mathrm{~s} ; 60^{\circ} \mathrm{C}, 45 \mathrm{~s} ; 72^{\circ} \mathrm{C}, 90 \mathrm{~s}\right)$, five amplification cycles $\left(94^{\circ} \mathrm{C}, 45 \mathrm{~s} ; 58^{\circ} \mathrm{C}, 45 \mathrm{~s} ; 72^{\circ} \mathrm{C}, 90 \mathrm{~s}\right), 30$ amplification cycles $\left(94{ }^{\circ} \mathrm{C}, 45 \mathrm{~s} ; 53^{\circ} \mathrm{C}\right.$ (gapdh) or $56{ }^{\circ} \mathrm{C}\left(\right.$ rpb2), $45 \mathrm{~s} ; 72{ }^{\circ} \mathrm{C}$, $90 \mathrm{~s}$ ) and a final extension step $\left(72^{\circ} \mathrm{C}, 5 \mathrm{~min}\right)$. PCR products were visualised by electrophoresis using a $1.2 \%$ agarose gel, stained with GelRed ${ }^{\mathrm{TM}}$ (Biotium, Hayward, CA) and viewed under ultra-violet light. Size estimates were made using a HyperLadder $^{\mathrm{TM}}$ I molecular marker (Bioline).

\section{Sequencing and phylogenetic analyses}

The resulting PCR fragments were sequenced in both directions using the same primers used for amplification (Table 2) and the BigDye Terminator Cycle Sequencing Kit v. 3.1 (Applied Biosystems, Foster City, CA), following the manufacturer's instructions. DNA sequencing amplicons were purified through Sephadex G-50 Superfine columns (SigmaAldrich, St Louis, MO) in 96-well MultiScreen HV plates (Millipore, Billerica, MA) as outlined by the manufacturer and analysed with an ABI Prism 3730xI Automated DNA analyser (Life Technologies Europe BV, Applied Biosystems ${ }^{\mathrm{TM}}$, Bleiswijk, The Netherlands).

The raw DNA sequences of tub2, gapdh and rpb2 were edited using MEGA v. 6 (Tamura et al. 2013) and forward and reverse sequences for each isolate were assembled manually to generate consensus sequences. Two parts of the rpb2 gene (part amplified with the fRPB2-5F/fRPB2414R primer set + part amplified with the RPB2-C5F/RPB2C8R primer set) were compiled manually using MEGA v. 6 . The assembled consensus sequences were initially aligned with MEGA v. 6 and optimised with the multiple sequence alignment online interface of MAFFT using default settings (http://mafft.cbrc.jp/alignment/server/) (Katoh \& Standley 2013), and adjusted manually where necessary. In addition, sequences of the same isolates corresponding to the ITS locus (including ITS1, 5.8S, ITS2), together with parts of four protein coding genes, viz. translation elongation factor 1-alpha (tef1), actin (act $A)$, calmodulin (cmdA) and histone $\mathrm{H} 3$ (his3), were retrieved from the NCBls GenBank nucleotide database and included in the analyses, after separate alignment as described above. Sequences of Cercospora sorghicola (CBS $136448=$ IRAN 2672C) were used as outgroup. Evolutionary models for phylogenetic analyses were selected independently for each locus using MrModeltest v. 2.3 (Nylander 2004) under the Akaike Information Criterion (AIC) (Table 3). The individual alignments of the different loci were subsequently concatenated with Mesquite v. 2.75 (Maddison \& Maddison 2011) prior to being subjected to a combined multi-gene analysis. Given the different sizes of the data partitions, they could not be properly used in statistical tests for (in) congruency. Phylogenetic reconstruction was performed using Bayesian inference (BI) Markov Chain Monte Carlo (MCMC) algorithm in MrBayes v. 3.2.2 (Ronquist et al. 2012). Two simultaneous MCMC analyses, each consisting of four Markov chains, were run from random trees until the average standard deviation of split frequencies reached a value of 0.01 , with trees saved every 100 generations and the heating parameter was set to 0.15 . Burn-in phase was set to $25 \%$ and the posterior probabilities (Rannala \& Yang 1996) were calculated from the remaining trees. The resulting phylogenetic tree was generated with Geneious v. 5.6.7 (Drummond et al. 2012).

All new sequences generated in this study were deposited in NCBls GenBank nucleotide database (www.ncbi.nlm.nih. gov; Table 1) and the alignment and phylogenetic trees in TreeBASE S22944 (www.TreeBASE.org).

\section{Morphology}

Morphological descriptions are based on structures from dried material. Diseased leaf tissues were viewed under a Nikon® SMZ1500 stereo-microscope and taxonomically informative morphological structures (stromata, conidiophores and conidia) were picked up from lesions with a sterile dissecting needle and mounted on glass slides in clear lactic acid. Structures were examined under a Nikon Eclipse 80i light microscope, and photographed using a mounted Nikon digital sight DS-f1 high definition colour camera.

Thirty measurements were made at $\times 1000$ for each microscopic structure, and $95 \%$ confidence intervals were derived for the measurements with extreme values given in parentheses. Colony macro-morphology on MEA was determined after $1 \mathrm{mo}$ at $25{ }^{\circ} \mathrm{C}$ in the dark in duplicate and colony colour was described using the mycological colour charts of Rayner (1970). Nomenclatural novelties and descriptions were deposited in MycoBank (www.mycobank. org; Crous et al. 2004). The naming system for tentatively applied names used by Groenewald et al. (2013) and Bakhshi et al. (2015a) is continued in this manuscript to simplify comparison between the studies. 


\section{Identification of the best-performing DNA barcode}

The dataset of the eight loci, ITS, tef1, actA, cmdA, his3, tub2, rpb2 and gapdh, was individually tested for two factors: Kimura-2-parameter (K2P) values (barcode gap) and molecular phylogenetic resolution (clade recovery). Inter- and intraspecific distances of eight loci were calculated for each single-locus sequence data alignment, using MEGA v. 6.0 with the Kimura-2-parameter distance values using the pairwise deletion model. Microsoft Excel 2010 was subsequently used to sort these distance values into distribution bins (from distance $0-0.1$ with intervals of 0.01 between bins) and the frequency of entries for each individual bin was then plotted against the Kimura-2-parameter distance of each bin.
In addition, Bayesian analyses using the corresponding nucleotide substitution models (Table 3 ) were applied to each data partition to check the stability and robustness of each species clade (clade recovery) under the different loci (data not shown, trees deposited in TreeBASE S22944) (Table 4). The clade recovery and Kimura-2-parameter values for each locus were calculated after applying the consolidated species concept to the results of eight-gene phylogenetic tree.

\section{Allele group designation}

The isolates in each of the Cercospora species complexes, including C. apii, C. armoraciae, C. beticola, C. cf. flagellaris, and Cercospora sp. G, were compared using the individual alignments of the eight single loci in MEGA v. 6. Allele groups

Table 4. Summary of clade support (Bayesian posterior probabilities (PP) values) for each species and locus or combination of loci. Green cells represent the PP values of species which are supported as distinct species, purple cells represent the PP values of species which are indistinct from one other species; while white cells represent species which cannot be distinguished from several other species for the given locus or combination of loci. The K2P inter-/intraspecies variation ratio as well as the number of species in the three different coloured categories are indicated per locus below the table. Abbreviations of loci follow Table 1.

\begin{tabular}{|c|c|c|c|c|c|c|c|c|c|}
\hline Locus/Loci & ITS & tef1 & act $A$ & $c m d A$ & his3 & tub2 & $r p b 2$ & gapdh & All 8 loci \\
\hline C. althaeina & & 1 & 0.91 & & 0.79 & 1 & 0.97 & 0.7 & 1 \\
\hline C. apii & & & & & & & & 1 & 1 \\
\hline C. armoraciae & & 0.73 & & 1 & 0.98 & 1 & $?$ & 1 & 1 \\
\hline C. beticola & & & & 1 & & & & 1 & 1 \\
\hline C. bizzozeriana & & 0.96 & & 1 & 0.98 & 1 & 1 & 1 & 1 \\
\hline C. chenopodii & 0.99 & 1 & 1 & 0.95 & 1 & 1 & 1 & 1 & 1 \\
\hline C. convolvulicola & & & 1 & 0.99 & & & & 1 & 1 \\
\hline C. conyzae-canadensis & & 0.96 & 1 & 0.92 & 1 & 1 & 1 & 0.57 & 1 \\
\hline C. cylindracea & & 1 & 0.9 & & 0.99 & 0.98 & 0.97 & 0.75 & 1 \\
\hline C. cf. flagellaris clade 1 & & & & & & & & 1 & 1 \\
\hline C. cf. flagellaris clade 2 & & & & & & & & 1 & 1 \\
\hline C. gamsiana & & & & 1 & & & & 1 & 1 \\
\hline C. cf. gossypii & & & & & & & & 1 & 1 \\
\hline C. iranica & & 1 & 0.94 & 1 & 1 & 0.98 & 1 & 0.91 & 1 \\
\hline C. plantaginis & & & & & & & & 1 & 1 \\
\hline C. pseudochenopodii & & 1 & 1 & 0.95 & 1 & 0.99 & 1 & 1 & 1 \\
\hline C. cf. richardiicola & & 1 & 1 & 1 & 1 & 1 & 1 & 1 & 1 \\
\hline C. rumicis & & 0.99 & & & 1 & & 1 & 1 & 1 \\
\hline C. solani & 1 & 0.99 & 0.93 & 0.83 & 1 & 1 & 1 & 1 & 1 \\
\hline C. sorghicola & 1 & 1 & 1 & 1 & 1 & 1 & 1 & 1 & 1 \\
\hline Cercospora sp. G clade 1 & & 0.98 & & 1 & 0.86 & 1 & 1 & 1 & 1 \\
\hline Cercospora sp. G clade 2 & & 0.98 & & 1 & 0.86 & 1 & 1 & 1 & 1 \\
\hline C. violae & & 0.97 & 1 & 0.93 & 0.94 & & 1 & 1 & 1 \\
\hline C. zebrina & & 0.73 & 0.87 & & 0.84 & & 1 & 0.75 & 1 \\
\hline C. cf. zinniae & & 1 & 1 & 1 & 1 & 1 & 1 & 1 & 1 \\
\hline $\mathrm{K} 2 \mathrm{P}$ inter-/intraspecies variation ratio & 4 & 127 & 15 & 76 & 13 & 71 & 74 & 44 & \\
\hline Number of distinct species & 3 & 11 & 12 & 9 & 13 & 12 & 9 & 17 & \\
\hline Number of two indistinct species & 0 & 8 & 2 & 8 & 6 & 4 & 8 & 11 & \\
\hline Number of unresolved species & 25 & 9 & 14 & 11 & 9 & 12 & 10 & 0 & \\
\hline
\end{tabular}


were established for each locus based on sequence identity, i.e. each sequence with one or more nucleotide difference from the other sequence was regarded as a different allele.

\section{RESULTS}

\section{DNA amplification and phylogenetic analysis}

New primers were designed for rpb2 and tub2 in this study (Table 2) and proved to be effective for the selected Cercospora species. Approximately 400, 1000, and 1200 bp were obtained for tub2, gapdh and rpb2 loci, respectively. The final concatenated eight-locus alignment contained 169 ingroup taxa and a total of 4099 characters including alignment gaps were processed. The gene boundaries were 1-470 bp for ITS, 475-765 bp for tef1, 770-956 bp for actA, 961-1 208 bp for cmdA, 1 213-1 570 bp for his3, 1 575-1 989 bp for tub2, 1994-3 222 bp for rpb2, and $3227-4099$ bp for gapdh. For the total alignment, 28 characters which were artificially introduced as spacers to separate the loci, were excluded from the phylogenetic analyses. The alignment contained 863 unique site patterns (Table 3).

The Bayesian analysis lasted 2405000 generations and generated 4812 trees from which the first 1202 trees (25\%), representing the burn-in phase of the analyses, were discarded, and the remaining trees (3 610) were used for calculating posterior probabilities (PP) values in the phylogenetic tree (50\% majority rule consensus tree) (Fig. 1).

\section{TAXONOMY}

Species delimitation in the genus Cercospora in this study follows the Consolidated Species Concept accepted in recent revisions of the taxonomy of cercosporoid fungi (e.g. Groenewald et al. 2013, Crous et al. 2013, Bakhshi et al. 2015a, Videira et al. 2017). Twenty-eight lineages of Cercospora were resolved based on the clustering and support in the Bayesian tree obtained from the combined ITS, tef1, actA, cmdA, his3, tub2, rpb2, and gapdh alignment (Fig. 1, Table 4). Of these, 15 species including C. althaeina, C. chenopodii, C. convolvulicola, C. conyzae-canadensis, C. cylindracea, C. iranica, C. pseudochenopodii, C. cf. richardiicola, C. rumicis, C. solani, C. sorghicola, Cercospora sp. T, C. violae, C. zebrina, and C. cf. zinnia, were the same as those also accepted before in the five-gene phylogenetic tree (ITS, tef1, actA, cmdA, and his3) (Bakhshi et al. 2015a). However, the eight-gene phylogenetic tree separated strains previously recognised as C. apii, C. armoraciae, C. beticola, C. cf. flagellaris, and Cercospora sp. G, based on fivegene phylogenetic tree (Groenewald et al. 2013, Bakhshi et al. 2015a) into at least three, two, two, four and two wellsupported clades respectively (Fig. 1). Some of these clades are supported by the host range or morphological characters of the isolates and are therefore described as new below.

\section{Cercospora apii complex}

The 16 isolates previously recognised as $C$. apii based on five-gene phylogenetic tree (Groenewald et al. 2013, Bakhshi et al. 2015a) are assigned here to three lineages based on the eight-gene phylogenetic tree, host association, and morphology, including C. apii s. str., C. uwebrauniana sp. nov., and C. plantaginis (Fig. 1, part 2). The results of allele group designation for the isolates in this complex detected one, four, two, two, four, three, four and two allele groups for the ITS, tef1, actA, cmdA, his3, tub2, rpb2, and gapdh sequences, respectively (Table 5).

Cercospora apii Fresen., Beitr. Mykol. 3: 91 (1863). Sensu Groenewald et al., Phytopathology 95: 954 (2005).

(Fig. 2)

Type: Germany: Oestrich, on Apium graveolens (Apiaceae), Fuckel, Fungi rhen. 117, in HAL (lectotype designated by Groenewald et al. 2005); Heilbronn, Landwirtschaftsamt, on A. graveolens, 10 Aug. 2004, K. Schrameyer (CBS $116455=$ CPC 11556 - epitype designated by Groenewald et al. 2005).

Description: Leaf spots amphigenous, distinct, circular to subcircular, 1-9 mm diam, white-grey in centre, surrounded by a dark purple-brown border. Mycelium internal. Caespituli amphigenous, brown. Conidiophores aggregated in moderately dense fascicles (4-15), arising from the upper cells of a well-developed brown stroma, to $50 \mu \mathrm{m}$ wide; conidiophores brown, becoming pale brown towards the apex, 1-6-septate, straight to variously curved, unbranched, uniform in wide, (45-)80-95(-125) × 4-5.5 $\mu \mathrm{m}$. Conidiogenous cells integrated, lateral or terminal, unbranched, brown, smooth, proliferating sympodially, $20-40 \times 3.5-5 \mu \mathrm{m}$, multi-local; loci thickened, darkened, refractive, apical or lateral, 2-3.5 $\mu \mathrm{m}$ diam. Conidia solitary, smooth, obclavate-cylindrical to acicular, straight to slightly curved, hyaline, distinctly 3-9(-15)-septate, apex subacute or subobtusely rounded, base subtruncate to obconically truncate, $(30-) 65-80(-115) \times 3-5 \mu \mathrm{m}$; hila thickened, darkened, refractive, 2-3.5 $\mu \mathrm{m}$ diam.

Note: This clade includes the ex-epitype strain of $C$. apii (isolate CBS $116455=$ CPC 11556), therefore we fixed the application of $C$. apii s. str. to this clade.

Specimens examined: Germany: Heilbron, Landwirtschaftsamt, on $A$. graveolens, K. Schrameyer (CBS 116455 = CPC 11556 -ex-epitype culture). - Iran: Ardabil Province: Moghan, on leaves of Cynanchum acutum (Apocynaceae), Oct. 2011, M. Bakhshi (IRAN 17016F, IRAN 17017F, CCTU 1069, CCTU 1086 = IRAN 2655C = CBS 136037); Moghan, on leaves of C. acutum, Oct. 2012, M. Bakhshi (IRAN 17018F, IRAN 17019F, CCTU 1215, CCTU 1219 = CBS 136155). - New Zealand: Auckland, on M. laevis, C.F. Hill (CPC 5112). - Romania: Bucuresti, on A. graveolens, 2 Oct. 1969, O. Constantinescu (CBS 536.71 = CPC 5087). - USA: California: on Moluccella laevis (Lamiaceae), S.T. Koike (CBS 110813 = CPC 5110).

Cercospora plantaginis Sacc., Michelia 1: 267 (1878). (Fig. 3)

Type: Italy: Selva, on Plantago lanceolata (Plantaginaceae), Sep. 1873, P.A. Saccardo (PAD, s.n. - holotype, according to Art. 9.1, Note 1). - Romania: Domnesti, on P. lanceolata, 3 Aug. 1965, O. Constantinescu (CBS 252.67 - epitype 


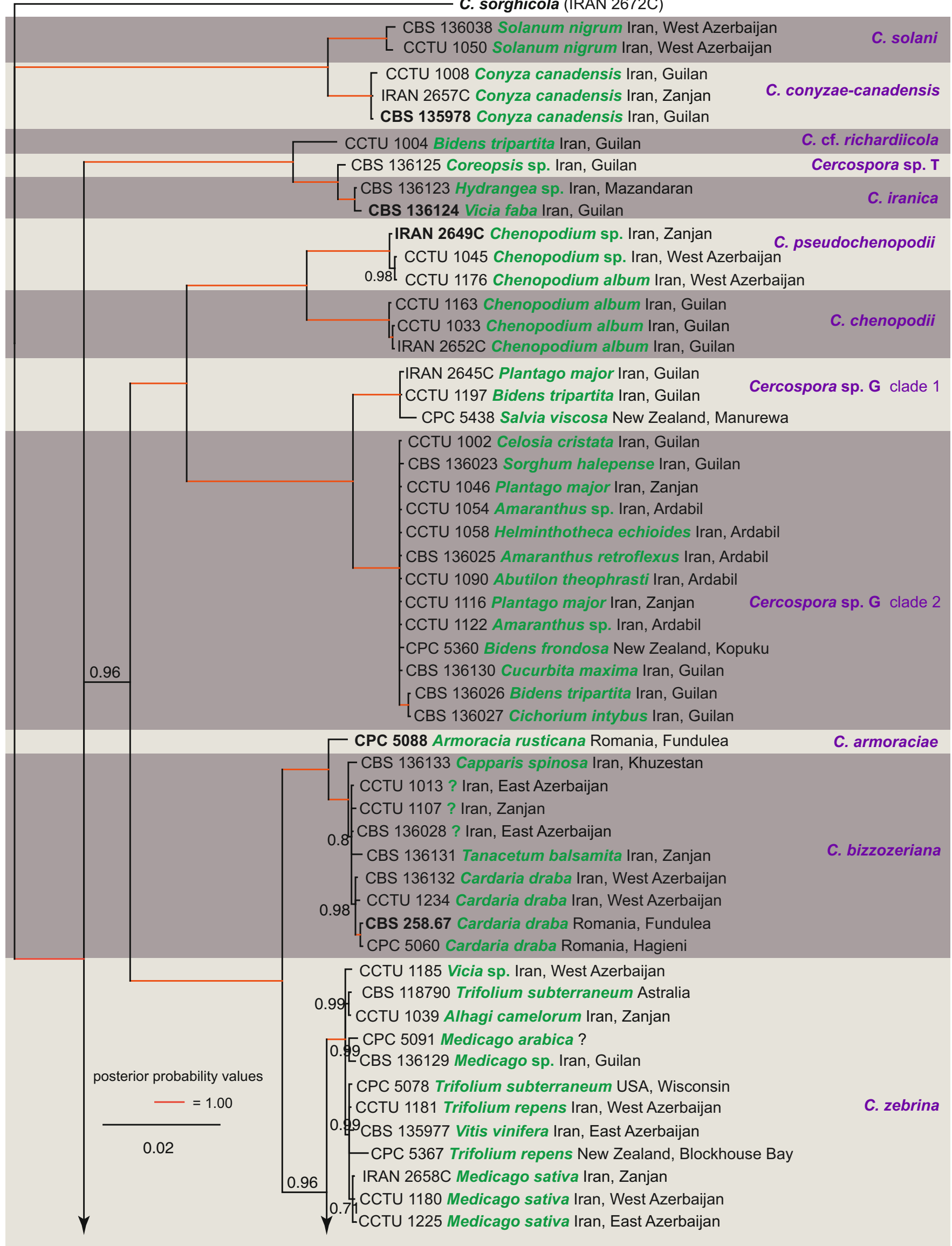

Fig. 1. Consensus phylogram ( $50 \%$ majority rule) of 3610 trees resulting from a Bayesian analysis of the combined eight-gene sequence alignment using MrBayes v. 3.2.2. The scale bar indicates 0.02 expected changes per site. Hosts and country of origin are indicated in green and black text, respectively. The tree was rooted to Cercospora sorghicola (isolate CBS $136448=$ IRAN 2672C). 


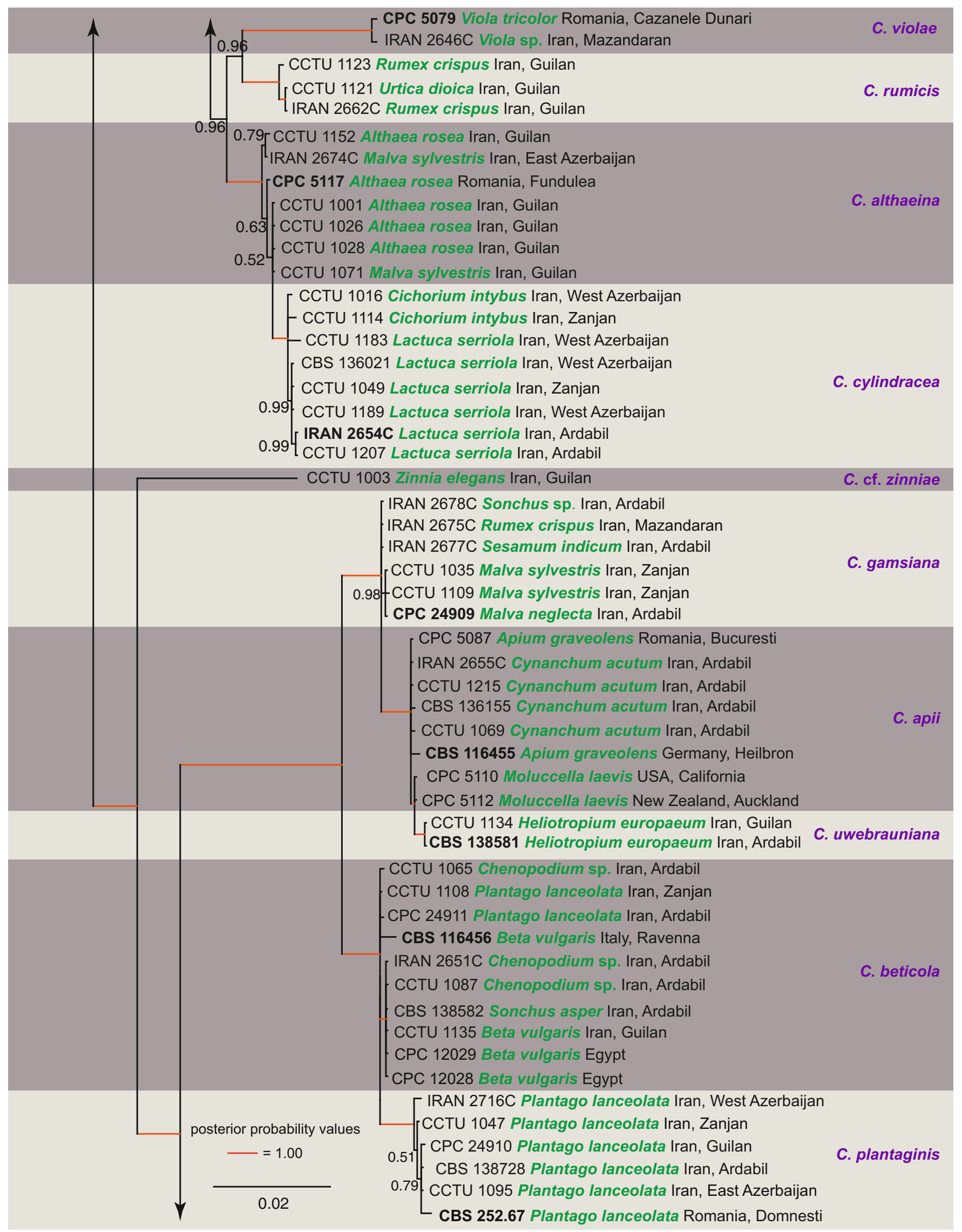

Fig. 1. (Continued). 


\begin{tabular}{|c|c|c|}
\hline$\uparrow$ & $\left\{\begin{array}{l}\text { IRAN 2650C Hibiscus trionum Iran, Ardabil } \\
\text { CBS } 136137 \text { Gossypium herbaceum Iran, Ardabil }\end{array}\right.$ & C. cf. gossypii \\
\hline 0.72 & $\begin{array}{l}\text { CPC } 5441 \text { Amaranthus sp. Fiji } \\
\text { CPC } 10884 \text { Dysphania ambrosioides South Korea, Jeju } \\
\text { - CBS } 136148 \text { Arachis hypogaea Iran, Guilan } \\
\text { CBS } 136031 \text { Hydrangea sp. Iran, Guilan } \\
\text { IRAN 2661C Phaseolus vulgaris Iran, Guilan } \\
\text { - CPC } 1051 \text { Populus deltoides South Aferica } \\
\text { CCTU } 1171 \text { Raphanus sativus Iran, Guilan } \\
\text { CCTU 1120 Raphanus sativus Iran, Guilan } \\
\text { IRAN 2648C Urtica dioica Iran, Guilan } \\
\text { IRAN 2670C Citrullus Ianatus Iran, Guilan } \\
\text { CBS 136034 Lepidium sativum Iran, Guilan } \\
\text { IRAN 2715C Phaseolus vulgaris Iran, Guilan }\end{array}$ & C. cf. flagellaris clade 1 \\
\hline 0.96 & 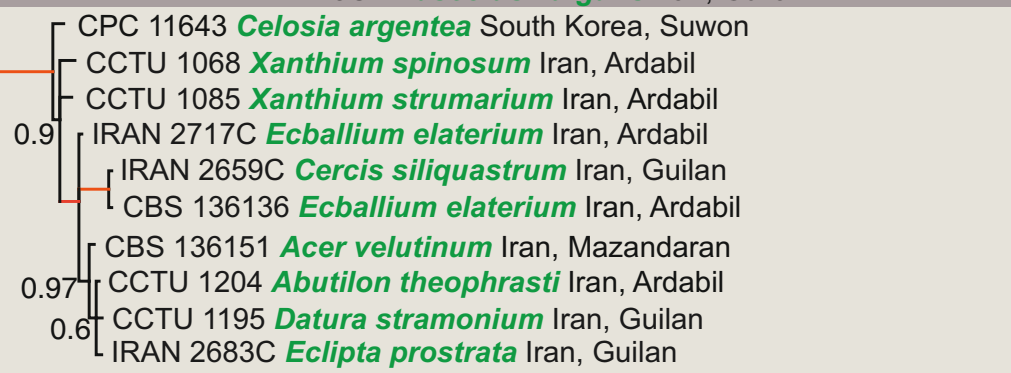 & C. cf. flagellaris clade 2 \\
\hline
\end{tabular}

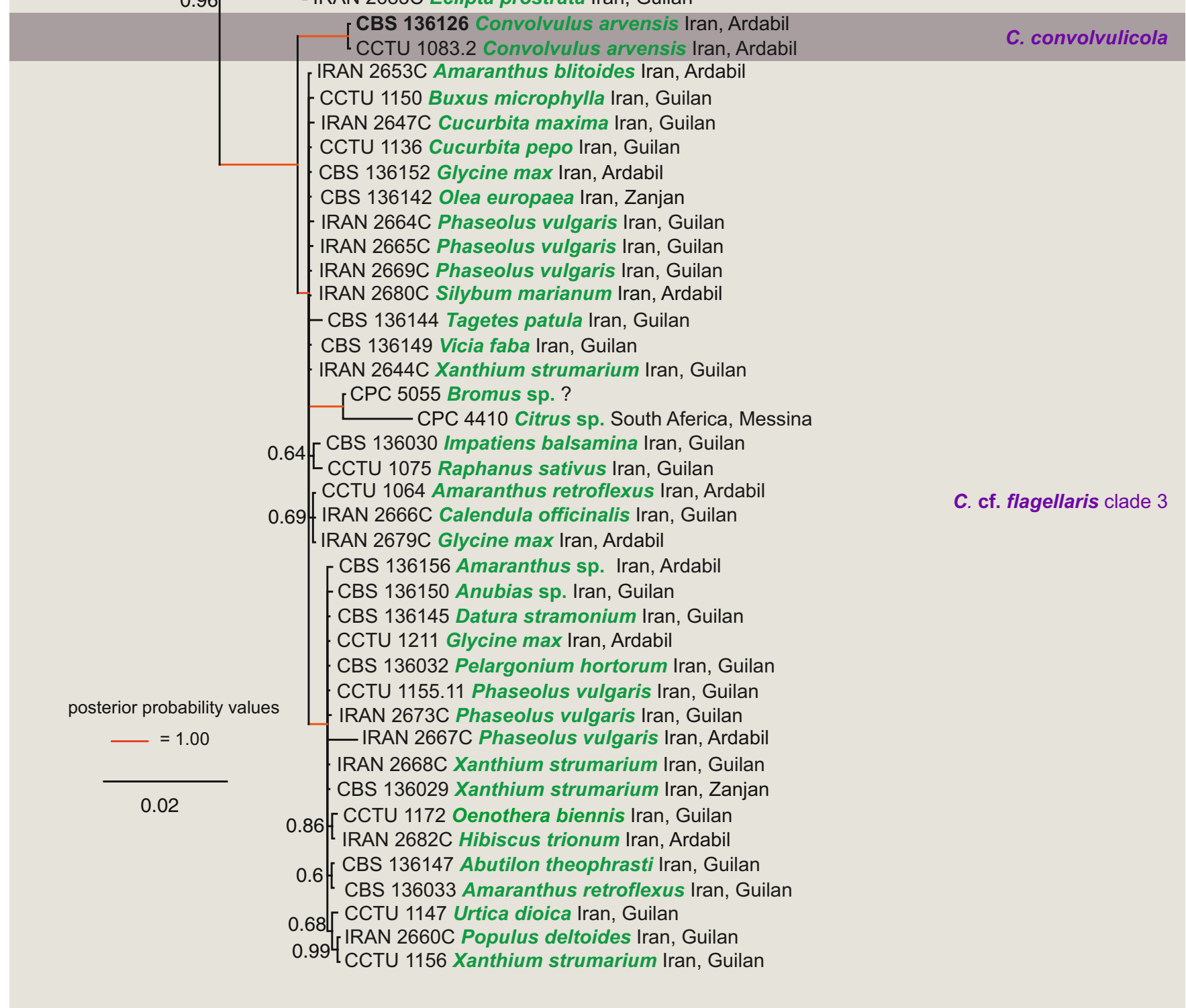

Fig. 1. (Continued). 
Table 5. Results from allele group designation per locus for Cercospora apii s. lat. isolates in this study. Abbreviations of loci and collection accession numbers follow Table 1.

\begin{tabular}{|c|c|c|c|c|c|c|c|c|c|c|}
\hline \multirow[t]{2}{*}{ Species } & \multirow[t]{2}{*}{ Culture accession number } & \multirow[t]{2}{*}{ Host } & \multicolumn{8}{|c|}{ Allele group per locus } \\
\hline & & & ITS & tef1 & actA & $c m d A$ & his3 & tub2 & rpb2 & gapdh \\
\hline \multirow[t]{8}{*}{ C. apii s. str. } & CCTU 1069 & Cynanchum acutum & $\overline{I I}$ & $\overline{\mathrm{II}}$ & 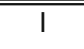 & $\bar{I}$ & 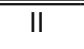 & $\overline{I I}$ & IV & $\bar{l}$ \\
\hline & $\begin{array}{l}\text { CCTU 1086; CBS 136037; IRAN } \\
2655 C\end{array}$ & Cynanchum acutum & 1 & II & 1 & 1 & II & I & I & 1 \\
\hline & CCTU 1215 & Cynanchum acutum & I & II & 1 & I & II & 1 & I & 1 \\
\hline & CCTU 1219; CBS 136155 & Cynanchum acutum & 1 & II & I & I & II & I & II & I \\
\hline & CBS 536.71; CPC 5087 & Apium graveolens & 1 & II & I & I & II & 1 & I & I \\
\hline & CBS 116455; CPC 11556 (TYPE) & Apium graveolens & I & 1 & I & I & I & I & - & I \\
\hline & CBS 110813; CPC 5110 & Molucella laevis & 1 & II & I & ॥ & II & 1 & 1 & 1 \\
\hline & CPC 5112 & Molucella laevis & I & II & I & II & II & I & I & I \\
\hline \multirow[t]{6}{*}{ C. plantaginis } & CCTU 1041; CPC 24910 & Plantago lanceolata & I & II & I & II & III & I & 1 & II \\
\hline & CCTU 1047 & Plantago lanceolata & I & II & I & II & II & II & I & II \\
\hline & CCTU 1082; CBS 138728 & Plantago lanceolata & 1 & II & I & II & III & II & 1 & II \\
\hline & CCTU 1095 & Plantago lanceolata & I & II & 1 & II & III & II & I & II \\
\hline & CCTU 1179 & Plantago lanceolata & I & II & II & II & II & 1 & III & II \\
\hline & CBS 252.67; CPC 5084 (TYPE) & Plantago lanceolata & I & III & II & II & III & II & _ & II \\
\hline \multirow[t]{2}{*}{ C. uwebrauniana } & CCTU 1134 & Heliotropium europaeum & I & IV & I & II & IV & III & I & I \\
\hline & CCTU 1200; CBS 138581 (TYPE) & Heliotropium europaeum & I & IV & I & II & IV & III & I & 1 \\
\hline
\end{tabular}
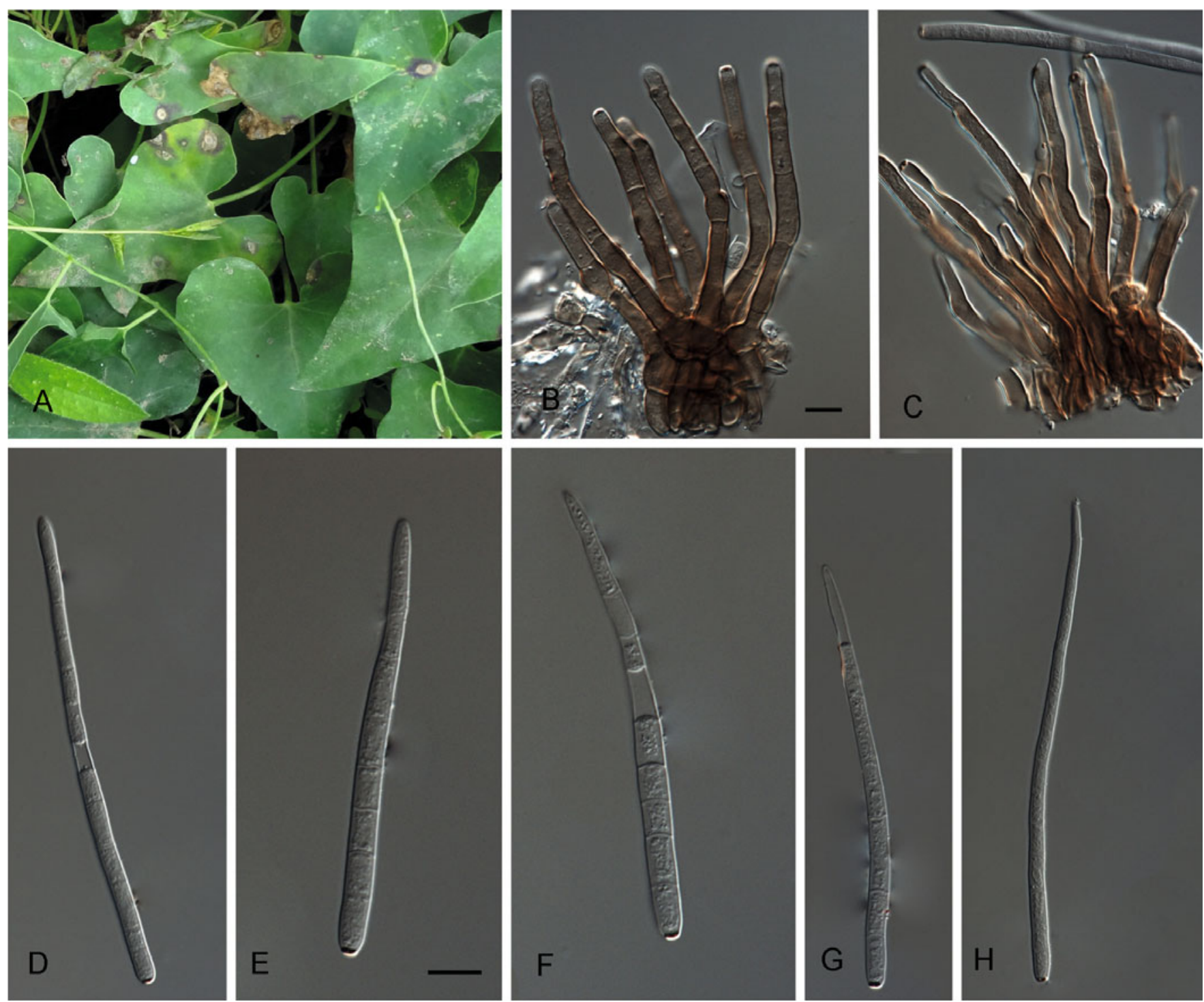

Fig. 2. Cercospora apii (CBS 136037). A. Leaf spots. B-C. Fasciculate conidiophores. D-H. Conidia. Bars $=10 \mu \mathrm{m}$. 


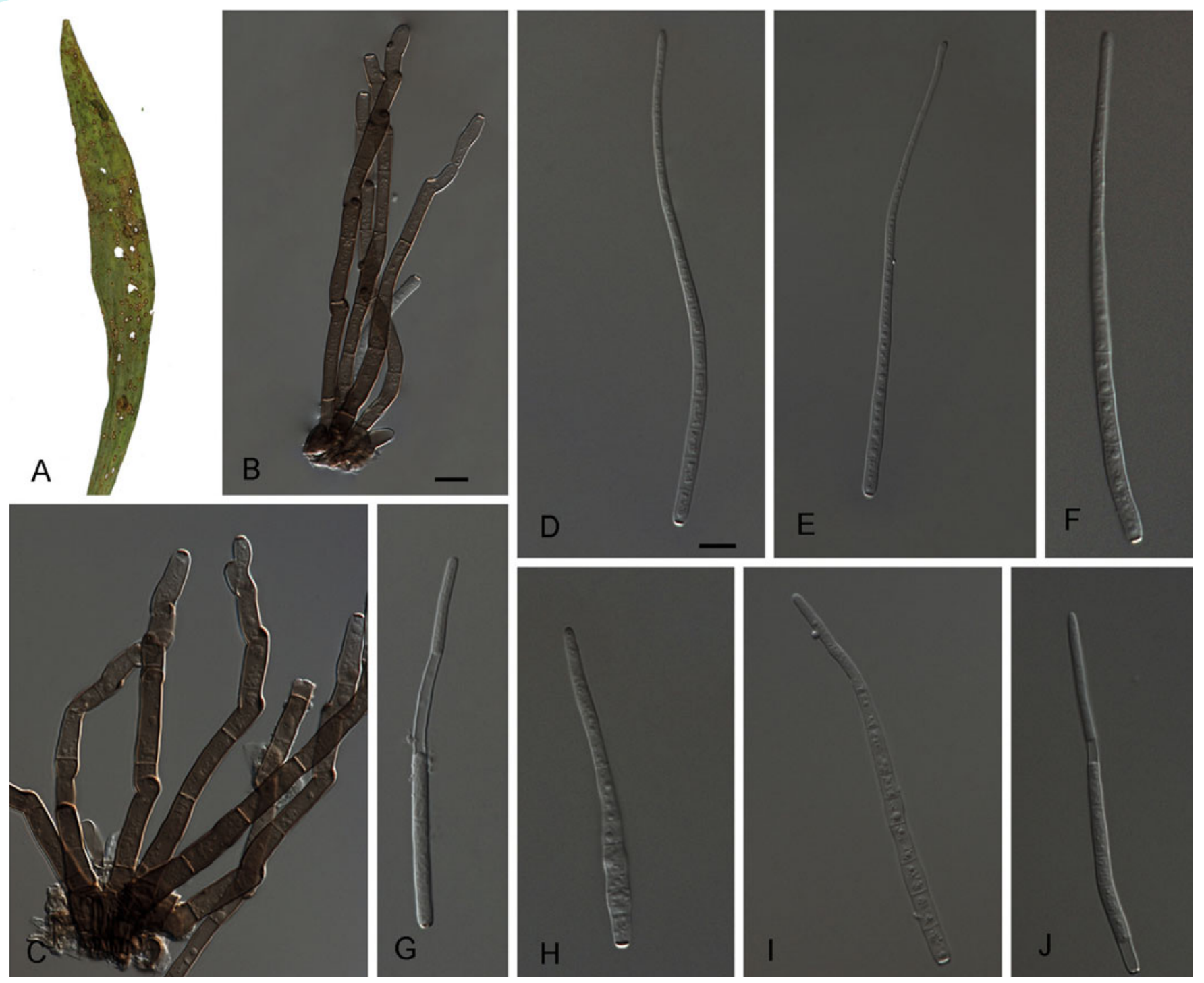

Fig. 3. Cercospora plantaginis (CPC 24910). A. Leaf spots. B-C. Fasciculate conidiophores. D-J. Conidia. Bars $=10 \mu \mathrm{m}$.

designated here, MBT 383093, preserved as a metabolically inactive culture).

Description: Leaf spots amphigenous, circular to subcircular, 1-4 $\mathrm{mm}$ diam, white to grey with distinct raised brown borders. Mycelium internal. Caespituli amphigenous, brown. Conidiophores aggregated in loose fascicles, arising from a moderately developed, intraepidermal and substomatal, dark brown stroma, to $30 \mu \mathrm{m}$ diam; conidiophores brown at the base, becoming paler towards the apex, 2-10-septate, straight to geniculate-sinuous due to sympodial proliferation, simple, uniform in width, somewhat constricted at the proliferating point, $(45-) 60-85 \times 4-5 \mu \mathrm{m}$. Conidiogenous cells integrated, terminal or lateral, pale brown to brown, proliferating sympodially, $8-25 \times 3.5-5 \mu \mathrm{m}$, multi-local; loci distinctly thickened, darkened and somewhat refractive, apical or formed on shoulders caused by sympodial proliferation, 2-3 $\mu \mathrm{m}$ diam. Conidia solitary, subcylindrical, filiform to acicular, straight to mildly curved, hyaline, (40-)60$70(-105) \times 2-3.5 \mu \mathrm{m},(4-) 8-13(-17)$-septate, with subobtuse to subacute apices and truncate bases; hila thickened, darkened, refractive, $1.5-2.5 \mu \mathrm{m}$ diam.
Notes: Based on the results of the eight-gene phylogenetic tree, all isolates obtained from $P$. lanceolata from five different provinces in Iran together with a European isolate from this host plant, previously recognised as $C$. apii based on a fivegene phylogenetic tree (Groenewald et al. 2013, Bakhshi et al. 2015a), cluster separately from the other isolates in this clade (Fig. 1, part 2). Three species of Cercospora, including C. apii, C. pantoleuca and C. plantaginis, have been reported from Plantago (Crous \& Braun 2003, https://nt.ars-grin.gov/ fungaldatabases/). This species is morphologically close to C. plantaginis described from Italy on P. lanceolata (Chupp 1954). Since one European isolate from $P$. lanceolata in Romania (CBS 252.67 = CPC 5084) also resides in this clade, we designate an epitype here for this species, and fix the application of the name C. plantaginis to this clade.

Additional specimens examined: Iran: Guilan Province: Chaboksar, on P. lanceolata, Jul. 2012, M. Bakhshi (IRAN 17076F, CCTU $1041=$ CPC 24910). Zanjan Province: Tarom, Pasar, on P. lanceolata, Sep. 2011, M. Bakhshi (IRAN 17078F, CCTU 1047). Ardabil Province: Moghan, on P. lanceolata, Sep. 2011, M. Bakhshi (CCTU $1082=$ CBS 138728). East Azerbaijan Province: Arasbaran, Horand, on $P$. 
lanceolata, Oct. 2011, M. Bakhshi (CCTU 1095). West Azerbaijan Province: Khoy, Firouragh, on P. lanceolata, Sep. 2012, M. Arzanlou (IRAN 17077F, CCTU $1179=$ IRAN 2716C).

\section{Cercospora uwebrauniana M. Bakhshi \& Crous, sp. nov.}

MycoBank MB827521

(Fig. 4)

Etymology: Named in honour of Uwe Braun, who has published extensively on the genus Cercospora, and also provided a modern treatment for allied genera of Mycosphaerellaceae.

Diagnosis: Differs from C. taurica in the cylindrical conidia with truncate or subtruncate bases and somewhat shorter and wider conidia, $(23-) 38-48(-70) \times 4.5-8 \mu \mathrm{m}$ vs $40-110 \times$ (2.5-)4-6(-7) $\mu \mathrm{m}$ in C. taurica.

Type: Iran: Ardabil Province: Moghan, on Heliotropium europaeum (Boraginaceae), Oct. 2012, M. Bakhshi (IRAN 16864F - holotype; CCTU 1200 = CBS 138581 - ex-type culture).

Description: Leaf spots distinct, circular to irregular, 3-10 $\mathrm{mm}$, grey-brown to dark brown, surrounded by brown margin. Mycelium internal. Caespituli amphigenous, brown. Conidiophores in moderately dense fascicles, arising from the upper cells of a moderately developed, intraepidermal and substomatal, brown stroma, to $40 \mu \mathrm{m}$ wide; conidiophores straight to slightly geniculate, pale brown to brown, unbranched, regular in width, $(60-) 115-145(-230) \times 3.5-5.5$ $\mu \mathrm{m}, 2-9$-septate. Conidiogenous cells integrated, terminal, brown, proliferating sympodially, $15-35 \times 3.5-5.5 \mu \mathrm{m}$, mostly mono-local, sometimes multi-local; loci distinctly thickened, darkened, refractive, apical or formed on the shoulders caused by geniculation, 2-3.5 $\mu \mathrm{m}$. Conidia solitary, hyaline, subcylindrical to cylindrical, straight or slightly curved, truncate to subtruncate at the base, obtuse to rounded at the apex, (23-)38-48(-70) × 4.5-8 $\mu \mathrm{m},(0-) 3-4(-9)$-septate; hila thickened, darkened, refractive, 1.5-3 $\mu \mathrm{m}$ diam.

Notes: Two isolates, obtained from $\mathrm{H}$. europaeum in different provinces in Iran, clustered in a small clade within $C$. apii s. str. (Fig. 1, part 2). This independent clade is supported by tef1, his 3 and tub2 from C. apii s. str. Morphologically, these two strains are completely distinct from their most closely related species in the phylogenetic tree, namely $C$. apii (conidia acicular, subacute or subobtusely rounded at the apex, $(30-) 65-80(-115) \times 3-5 \mu \mathrm{m})$, C. beticola (conidia subacute to acute apex, $(40-) 90-140(-300) \times 2-5 \mu \mathrm{m})$, C. gamsiana (conidia subobtuse at the apex, (27-)49-62(-100) × 2-4 $\mu \mathrm{m}$ ) and $C$. plantaginis (conidia subobtuse to subacute apices, $(40-) 60-70(-105) \times 2-3.5 \mu \mathrm{m})$, by the obtuse to rounded apex, wider and shorter conidia $((23-) 38-48(-70) \times 4.5-8$ $\mu \mathrm{m})$, and are regarded as a separate species, appearing to be confined to $H$. europaeum.

Presently, three species of Cercospora have been described from Heliotropium, C. apii, C. heliotropiicola, and C. taurica (Crous \& Braun 2003, https://nt.ars-grin.gov/fungaldatabases/). Cercospora uwebrauniana differs from C. taurica in the cylindrical conidia with truncate or subtruncate bases and somewhat shorter and wider conidia, (23-)38-48(-70) $\times$ $4.5-8 \mu \mathrm{m}$ vs 40-110 × (2.5-)4-6(-7) $\mu \mathrm{m}$ in C. taurica (Braun 2002). In addition, $C$. taurica has obclavate-cylindrical conidia with obconically truncate bases and rather wider conidiophores, 4-9 $\mu \mathrm{m}$ diam (Braun 2002). Cercospora heliotropiicola is morphologically quite distinct from $C$. uwebrauniana in having acicular or subulate, much thinner (2-3 $\mu \mathrm{m}$ wide) and longer (to $300 \mu \mathrm{m}$ long) conidia with subobtuse or acute apex (Pons \& Sutton 1996).

Additional specimen examined: Iran: Guilan Province: Astara, on $H$. europaeum, Jun. 2012, M. Bakhshi (IRAN 17096F, CCTU 1134).

\section{Cercospora armoraciae complex}

The 10 isolates previously recognised as C. armoraciae based on a five-gene phylogenetic tree (Groenewald et al. 2013, Bakhshi et al. 2015a) are assigned to two lineages here, based on the eight-gene phylogenetic tree, including C. armoraciae s. str. and C. bizzozeriana (Fig. 1, part 1). The results of allele group designation for the isolates in this complex revealed one, three, one, two, seven, three, three and two allele groups for the ITS, tef1, actA, cmdA, his3, tub2, rpb2 and gapdh sequences, respectively (Table 6).

Cercospora armoraciae Sacc., Nuovo Giorn. Bot. Ital. 8: 188 (1876).

Note: This clade includes the ex-type culture of $C$. armoraciae (CBS 250.67).

Cercospora bizzozeriana Sacc. \& Berl., Malpighia 2: 248 (1888).

(Fig. 5)

Type: Italy: Padova, on Lepidium latifolium (Brassicaceae), (Berlese, Malpighia 1: tab. XIV, fig. 23, 1887 - lectotype, designated here, MBT 383343); Romania: Fundulea, on Cardaria draba, isol. by $O$. Constantinescu [deposited in the CBS culture collection in 1967] (CBS 258.67 - epitype designated here, MBT 383154, preserved as a metabolically inactive culture).

Notes: Type material of C. bizzozeriana is not preserved in Saccardo's herbarium (see Gola 1930). Therefore, the original illustration published by Saccardo \& Berlese (in Berlese 1888) is designated as lectotype (according to Art. 9.3 and 9.4). Berlese's article "Fungi veneti novi vel critici" was split into several parts published in Malpighia 1 (1887) and 2 (1888). The description of $C$. bizzozeriana was published in vol 2, but with reference to tab. XIV, fig. 23 already issued in vol. 1.

Description: Leaf spots amphigenous, circular, 1-5 mm, white to white-grey with grey to black dots (stroma with conidiophores) and definite brown border. Mycelium internal. Caespituli amphigenous, brown. Conidiophores aggregated in dense fascicles, arising from a well-developed, brown stroma, to $75 \mu \mathrm{m}$ diam; conidiophores brown, 1-5-septate, straight to geniculate-sinuous due to sympodial proliferation, simple, sometimes branched, uniform in width, sometimes 

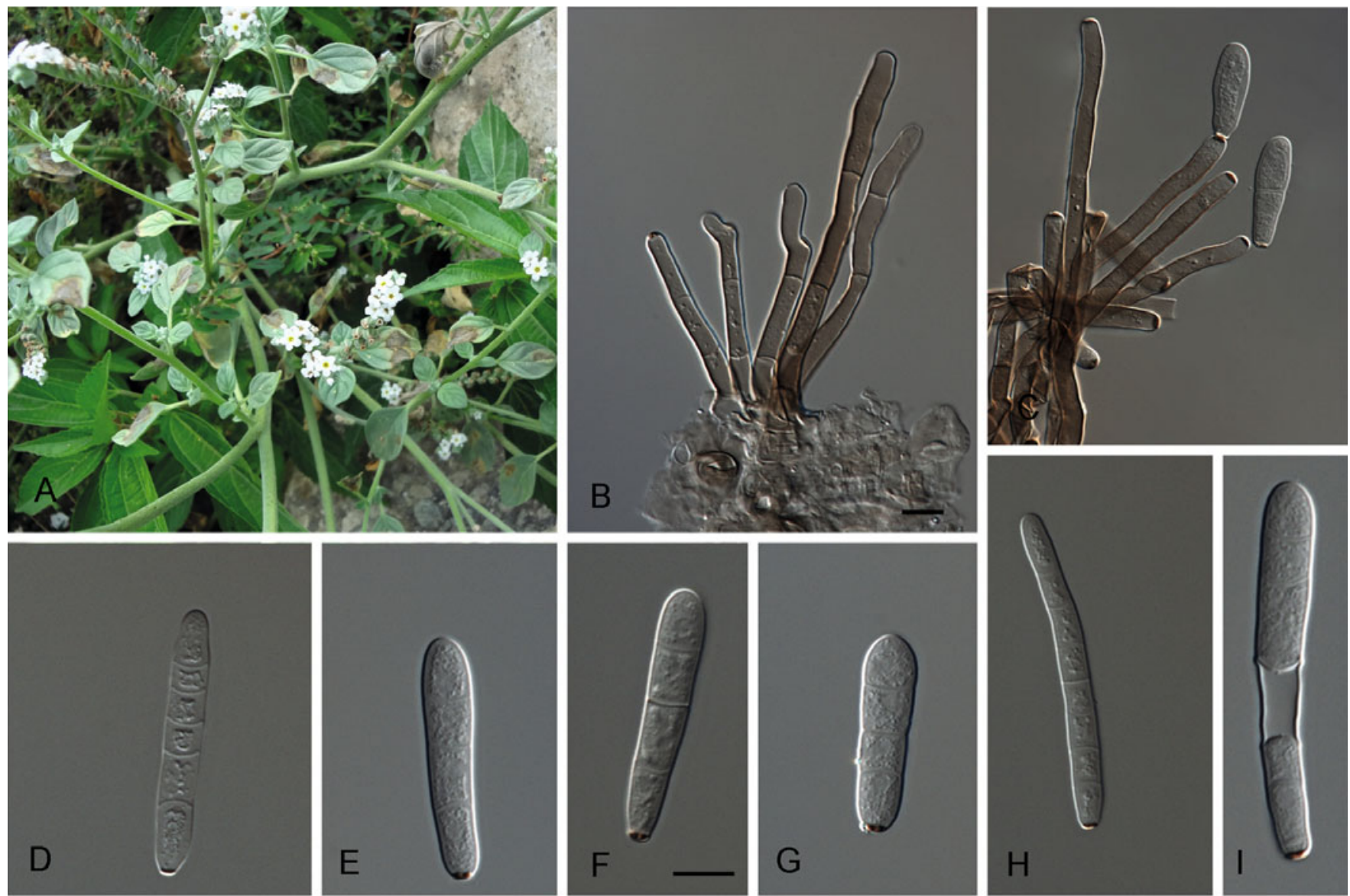

Fig. 4. Cercospora uwebrauniana (CBS 138581). A. Leaf spots. B-C. Fasciculate conidiophores. D-I. Conidia. Bars $=10 \mu \mathrm{m}$.

constricted at the proliferating point, $(30-) 50-60(-80) \times 4-7$ $\mu \mathrm{m}$. Conidiogenous cells integrated, terminal or lateral, pale brown to brown, proliferating sympodially, 10-25 × 3-6 $\mu \mathrm{m}$, multi-local; loci distinctly thickened, darkened and somewhat refractive, apical, lateral or formed on shoulders caused by geniculation, 1.5-3 $\mu \mathrm{m}$ diam. Conidia solitary, obclavatecylindrical, straight to slightly curved, hyaline, (20-)60$80(-125) \times 3-6 \mu \mathrm{m}, 2-10$-septate, with obtuse apices and subtruncate or obconically truncate bases; hila thickened, darkened, refractive, $1.5-3 \mu \mathrm{m}$ diam.
Notes: Isolates obtained from different host species including Tanacetum balsamita, Capparis spinosa and Cardaria draba clustered in a clade distinct from the ex-type isolate of $C$. armoraciae, and are regarded as a separate taxon. In addition, five isolates obtained from Car. draba (three from Iran and two from Romania) all cluster in this clade. Until now, three species of Cercospora are known from these host species, including C. bizzozeriana, C. chrysanthemi and C. capparis (Crous \& Braun 2003, https://nt.ars-grin.gov/ fungaldatabases/). Cercospora chrysanthemi is in the C. apii

Table 6. Results from allele group designation per locus for Cercospora armoraciae s. lat. isolates in this study. Abbreviations of loci and collection accession numbers follow Table 1.

\begin{tabular}{|c|c|c|c|c|c|c|c|c|c|c|}
\hline \multirow[t]{2}{*}{ Species } & \multirow{2}{*}{$\begin{array}{l}\text { Culture accession } \\
\text { number }\end{array}$} & \multirow[t]{2}{*}{ Host } & \multicolumn{8}{|c|}{ Allele group per locus } \\
\hline & & & ITS & tef1 & actA & cmdA & his3 & tub2 & $r p b 2$ & gapdh \\
\hline C. armoraciae s. str. & $\begin{array}{l}\text { CBS 250.67; CPC } 5088 \\
\text { (TYPE) }\end{array}$ & $\begin{array}{l}\text { Armoracia rusticana } \\
(=A \text {. lapathifolia) }\end{array}$ & 1 & I & 1 & 1 & I & 1 & - & II \\
\hline \multirow[t]{9}{*}{ C. bizzozeriana } & CCTU 1013 & $?$ & 1 & II & 1 & 1 & III & 1 & I & I \\
\hline & CCTU 1022; CBS 136028 & $?$ & 1 & II & 1 & 1 & III & 1 & I & I \\
\hline & CCTU 1040; CBS 136131 & Tanacetum balsamita & 1 & III & 1 & II & $\mathrm{VI}$ & 1 & II & I \\
\hline & CCTU 1107 & $?$ & 1 & II & 1 & I & VII & 1 & I & 1 \\
\hline & CCTU 1117; CBS 136132 & Cardaria draba & I & II & I & I & V & 1 & I & I \\
\hline & CCTU 1234 & Cardaria draba & I & II & I & I & $\mathrm{V}$ & III & I & I \\
\hline & CCTU 1127; CBS 136133 & Capparis spinosa & 1 & II & 1 & 1 & IV & II & III & 1 \\
\hline & CBS 540.71; CPC 5060 & Cardaria draba & 1 & II & 1 & 1 & II & 1 & - & 1 \\
\hline & $\begin{array}{l}\text { CBS 258.67; CPC } 5061 \\
\text { (TYPE) }\end{array}$ & Cardaria draba & 1 & II & I & 1 & II & 1 & - & 1 \\
\hline
\end{tabular}



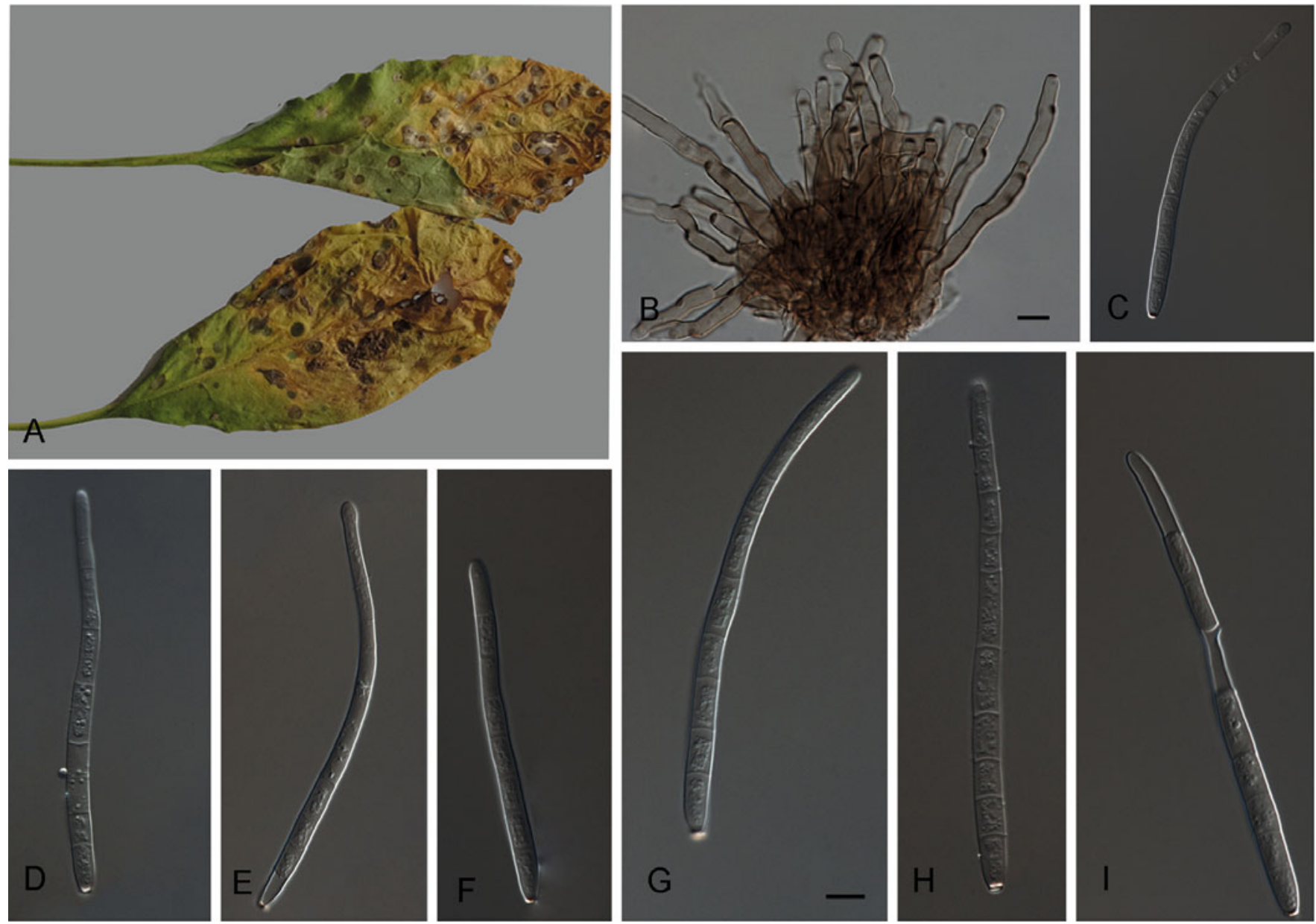

Fig. 5. Cercospora bizzozeriana (CBS 136132). A-B. Leaf spots. C. Fasciculate conidiophores. D-J. Conidia. Bars $=10 \mu \mathrm{m}$.

s. lat. complex (Crous \& Braun 2003). Cercospora capparis differs from this species by the narrower $(4-5.5 \mu \mathrm{m}$ diam) conidiophores and 3-5 $\mu \mathrm{m}$ diam conidia (Chupp 1954). The species is morphologically close to C. bizzozeriana which was described from Italy on Car. draba (Chupp 1954). Since two European isolates from Car. draba in Romania also reside in this clade, we designate an epitype here (ex-epitype culture CBS $258.67=$ CPC 5061) for this species, and fix the application of $C$. bizzozeriana to this clade.

Additional specimens examined: Iran: West Azerbaijan Province: Khoy, Firouragh, on leaves of Car. draba, Nov. 2011, M. Arzanlou (CCTU 1117 = CBS 136132); Khoy, Firouragh, on leaves of Car. draba, Oct. 2012, M. Arzanlou (IRAN 17027F, CCTU 1234). Zanjan Province: Tarom, Haroun Abad, on leaves of Tanacetum balsamita (Asteraceae), Sep. 2011, M. Bakhshi (IRAN 17029F, CCTU 1040 = CBS 136131); Tarom, Mamalan, Oct. 2011, M. Bakhshi (IRAN 17028F, CCTU 1107); Mianeh, Oct. 2012, M. Torbati (IRAN 17025F, IRAN 17026F, CCTU 1013, CCTU 1022 = CBS 136028). Khuzestan Province: Ahvaz, on leaves of Capparis spinosa (Capparidaceae), Dec. 2011, E. Mohammadian (CCTU 1127 = CBS 136133). Romania: Hagieni, on Car. draba, O. Constantinescu (CBS $540.71=$ IMI $161110=$ CPC 5060).

\section{Cercospora beticola complex}

The 16 isolates previously recognised as $C$. beticola based on a five-gene phylogenetic analysis (Groenewald et al.
2013, Bakhshi et al. 2015a), are assigned to two lineages based on the eight-gene phylogenetic analysis (Fig. 1, part 2). One, one, one, one, one, two, three and four allele groups were distinguished for the ITS, tef1, actA, cmdA, his3, tub2, rpb2 and gapdh sequences, respectively (Table 7).

Cercospora beticola Sacc., Nuovo Giorn. Bot. Ital. 8: 189 (1876).

Sensu Groenewald et al., Phytopathology 95: 954 (2005).

(Fig. 6)

Type: Italy: Vittorio (Treviso), on Beta vulgaris (Chenopodiaceae), Sep. 1897, P.A. Saccardo, Fungi ital. no. 197 (PAD - neotype designated by Groenewald et al. 2005); Ravenna, on B. vulgaris, 10 Jul. 2003, V. Rossi (CBS $116456=$ CPC 11557 - epitype designated by Groenewald et al. 2005).

Description: Leaf spots amphigenous, distinct, circular to subcircular, 1-7 $\mathrm{mm}$ diam, white-grey, with grey dots (stroma with conidiophores), surrounded by distinct brown border. Mycelium internal. Caespituli amphigenous, brown. Conidiophores aggregated in loose to dense fascicles, emerging through stomatal openings or erumpent through the cuticle, arising from the upper cells of a moderately to well-developed brown stroma, to $110 \mu \mathrm{m}$ diam; conidiophores brown, becoming paler towards apex, 2-8-septate, thick- 


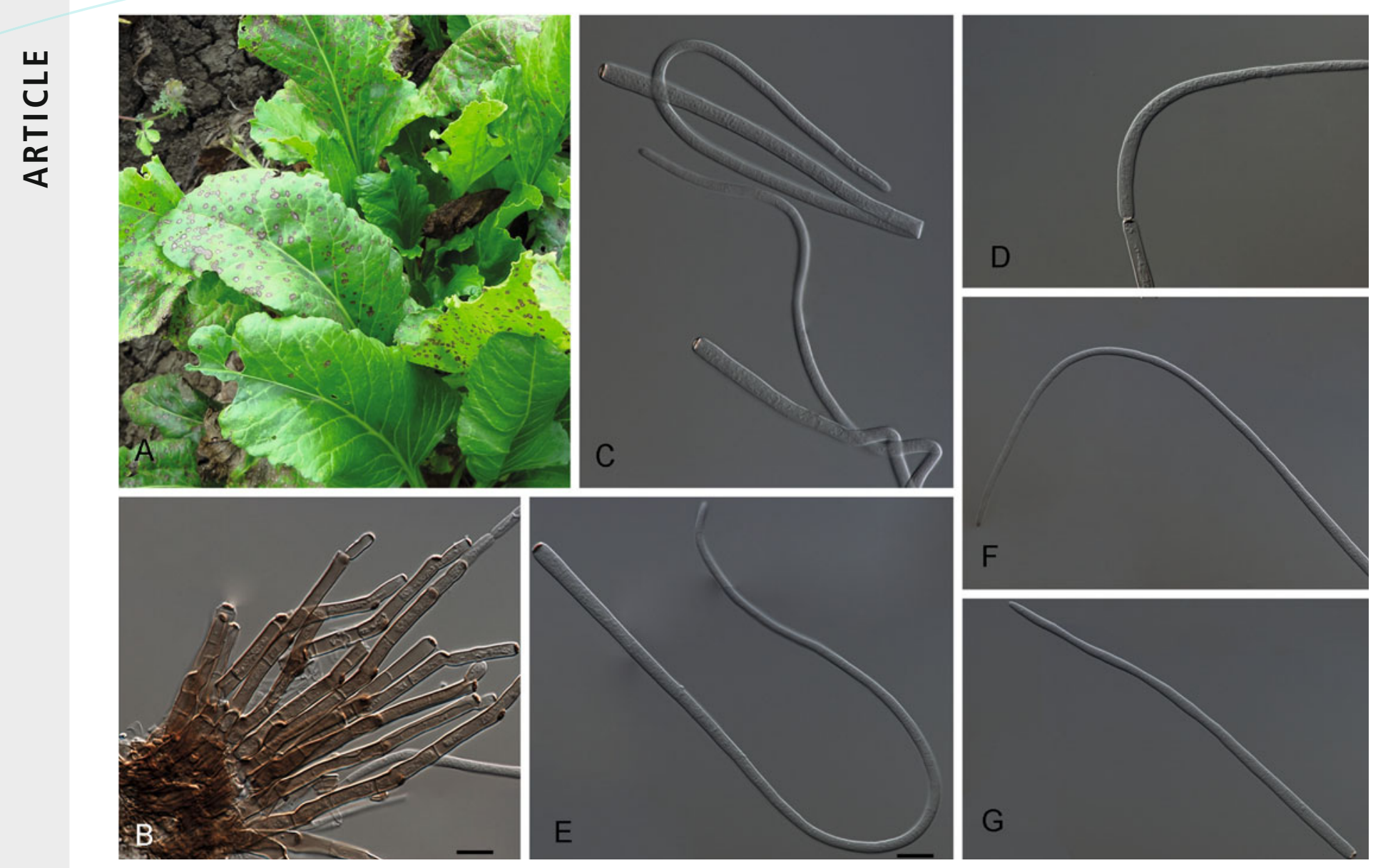

Fig. 6. Cercospora beticola (CCTU 1135). A. Leaf spots. B. Fasciculate conidiophores. C-G. Conidia. Bars $=10 \mu \mathrm{m}$.

Table 7. Results from allele group designation per locus for Cercospora beticola s. lat. isolates in this study. Abbreviations of loci and collection accession numbers follow Table 1 .

\begin{tabular}{|c|c|c|c|c|c|c|c|c|c|c|}
\hline \multirow[t]{2}{*}{ Species } & \multirow[t]{2}{*}{ Culture accession number } & \multirow[t]{2}{*}{ Host } & \multicolumn{8}{|c|}{ Allele group per locus } \\
\hline & & & ITS & tef1 & $\operatorname{act} A$ & $c m d A$ & his3 & tub2 & rpb2 & gapdh \\
\hline \multirow[t]{10}{*}{ C. beticola } & CCTU 1057; IRAN 2651C & Chenopodium sp. & 1 & 1 & 1 & 1 & 1 & 1 & II & III \\
\hline & CCTU 1065 & Chenopodium sp. & 1 & 1 & I & 1 & 1 & I & II & II \\
\hline & CCTU 1087 & Chenopodium sp. & 1 & I & 1 & 1 & 1 & I & II & III \\
\hline & CCTU 1088; CBS 138582 & Sonchus asper & 1 & I & 1 & 1 & 1 & I & II & III \\
\hline & CCTU 1089; CPC 24911 & Plantago lanceolata & 1 & I & 1 & 1 & 1 & II & II & II \\
\hline & CCTU 1108 & Plantago lanceolata & I & 1 & I & 1 & 1 & 1 & II & II \\
\hline & CBS 116456; CPC 11557 (TYPE) & Beta vulgaris & 1 & I & I & 1 & 1 & 1 & 1 & I \\
\hline & CCTU 1135 & Beta vulgaris & 1 & I & I & 1 & 1 & 1 & II & III \\
\hline & CPC 12028 & Beta vulgaris & 1 & I & I & 1 & 1 & I & II & III \\
\hline & CPC 12029 & Beta vulgaris & 1 & I & I & 1 & 1 & 1 & $\underline{I}$ & III \\
\hline \multirow[t]{6}{*}{ C. gamsiana } & CCTU 1035 & Malva sylvestris & I & 1 & 1 & I & 1 & 1 & III & IV \\
\hline & $\begin{array}{l}\text { CBS 144962; CCTU 1074; CPC } 24909 \\
\text { (TYPE) }\end{array}$ & Malva neglecta & 1 & 1 & 1 & 1 & 1 & 1 & III & IV \\
\hline & CCTU 1109 & Malva sylvestris & 1 & 1 & 1 & 1 & 1 & 1 & III & IV \\
\hline & CCTU 1199; CBS 136128; IRAN 2675C & Rumex crispus & 1 & I & 1 & 1 & 1 & 1 & II & IV \\
\hline & CCTU 1205; CBS 136127; IRAN 2677C & Sesamum indicum & 1 & I & 1 & 1 & 1 & 1 & II & IV \\
\hline & CCTU 1208; IRAN 2678C & Sonchus sp. & I & I & 1 & I & 1 & 1 & II & IV \\
\hline
\end{tabular}

walled, straight to geniculate-sinuous, unbranched, uniform in width, $(30-) 80-110(-185) \times 4-5(-6) \mu \mathrm{m}$. Conidiogenous cells integrated, terminal or lateral, unbranched, brown, smooth, proliferating sympodially, $10-30 \times 3.5-5.5 \mu \mathrm{m}$, mostly multi-local, sometimes mono-local; loci apical or formed on shoulders caused by geniculation, thickened, darkened, 
refractive, 1.5-2 $\mu \mathrm{m}$ diam. Conidia solitary, subcylindrical, filiform to acicular, straight to variously curved, hyaline, 3-15(-29)-septate, apex subacute to acute, base truncate to subtruncate, (40-)90-140(-300) × 2-5 $\mathrm{mm}$; hila thickened, darkened, refractive, 1.5-2.5 $\mu \mathrm{m}$ diam.

Note: This clade includes the ex-epitype culture of $C$. beticola (CBS $116456=$ CPC 11557), therefore we fixed the application of the name $C$. beticola s. str. to this clade.

Additional specimens examined: Egypt, on B. vulgaris, 15 Apr. 2004, M. Hasem (CPC 12028, CPC 12029). - Iran: Guilan Province: Talesh, Khotbeh Sara, on leaves of B. vulgaris, Jun. 2012, M. Bakhshi (IRAN 17020F, CCTU 1135). Zanjan Province: Tarom, Mamalan, on P. lanceolata, Oct. 2011, M. Bakhshi (IRAN 17023F, CCTU 1108). Ardabil Province: Moghan, on P. lanceolata, Oct. 2011, M. Bakhshi (CCTU 1089 = CPC 24911); Moghan, on Chenopodium sp. (Chenopodiaceae), Oct. 2011, M. Bakhshi (IRAN 17021F, IRAN 17022F, CCTU 1057 = IRAN 2651C, CCTU 1065, CCTU 1087); Moghan, on Sonchus asper (Asteraceae), Oct. 2011, M. Bakhshi (IRAN 17024F, CCTU 1088 = CBS 138582).

Cercospora gamsiana M. Bakhshi \& Crous, sp. nov. MycoBank MB827522

(Fig. 7)

Etymology: Dedicated to the recently deceased Walter Gams to honour his contribution to mycology.

Diagnosis: Morphologically distinct from species of the $C$. apii complex in the irregularly constricted, often conical and attenuated at the apex conidiophores, and conidia with long obconically truncate bases; sporulation is restricted to the terminal part of conidiophores.

Type: Iran: Ardabil Province: Moghan, on leaves of Malva neglecta (Malvaceae), Oct. 2011, M. Bakhshi (IRAN 17011F - holotype; CBS 144962 = CCTU 1074 = CPC 24909- extype culture).

Description: Leaf spots amphigenous, circular to irregular, 3-8 mm diam, grey to brown. Mycelium internal. Caespituli amphigenous, brown. Conidiophores aggregated in moderately dense fascicles, arising from a well-developed, intraepidermal and substomatal, brown stroma, to $45 \mu \mathrm{m}$ diam; conidiophores pale brown, 1-5-septate, geniculatesinuous, irregularly constricted, unbranched, moderately thin-walled, irregular in width, often conical and attenuated at the apex, sporulation is restricted at the terminal part of conidiophores, 45-60(-110) × 4-5 $\mu \mathrm{m}$. Conidiogenous cells integrated, terminal, pale brown to olivaceous-brown, proliferating sympodially, $10-25 \times 3.5-5 \mu \mathrm{m}$, uni- or multilocal; loci distinctly thickened, darkened and somewhat refractive, apical, circumspersed, 1.5-2 $\mu \mathrm{m}$ diam. Conidia solitary, subcylindrical to obclavate or somewhat narrowed towards the tip, straight to slightly curved, hyaline, thinwalled, (27-)49-62(-100) × 2-4 $\mu \mathrm{m}$, distinctly 3-10-septate, subobtuse at the apex and long obconically truncate at the base; hila distinctly thickened, darkened, refractive, 1.5-2.5 $\mu \mathrm{m}$ diam.
Notes: Until now, 14 species names in Cercospora have been introduced from these host species, including $C$. apii, C. althaeina, C. beticola, C. hyalospora (C. apii s. lat. complex), C. malvarum (C. apii s. lat. complex), C. malvicola, C. sigesbeckiae, C. peckiana (C. apii s. lat. complex), C. rumicis, C. sonchi (C. apii s. lat. complex), C. sonchicola (C. apii s. lat. complex), C. sonchifolia, C. sesami (C. apii s. lat. complex), and C. sesamigena (Crous \& Braun 2003, https:// nt.ars-grin.gov/fungaldatabases/). Cercospora gamsiana is phylogenetically clearly distinguishable from C. apii, C. althaeina, C. beticola, C. sigesbeckiae and C. rumicis (Bakhshi et al. 2015a) (Fig. 1, part 2). It is morphologically well distinguished from species of the $C$. apii complex and other species of Cercospora by its irregularly constricted, thinwalled, often conical and attenuated at the apex conidiophores and, conidia with long obconically truncate bases; sporulation is restricted at the terminal part of conidiophores.

Additional specimens examined: Iran: Zanjan Province: Tarom, Zehtar Abad, on leaves of Malva sylvestris, Sep. 2011, M. Bakhshi (CCTU 1035); Tarom, Mamalan, on leaves of M. sylvestris, Oct. 2011, M. Bakhshi (CCTU 1109). Ardabil Province: Moghan, on leaves of Sonchus sp., Oct. 2012, M. Bakhshi (IRAN 17072F, CCTU $1208=$ IRAN 2678C); Moghan, on leaves of Sesamum indicum (Pedaliaceae), Oct. 2012, M. Bakhshi (CCTU 1205 = IRAN 2677C = CBS 136127). Guilan Province: Ramsar, on leaves of Rumex crispus (Polygonaceae), Sep. 2012, M. Bakhshi (CCTU 1199 = IRAN 2675C = CBS 136128).

\section{Cercospora cf. flagellaris complex}

The 61 isolates previously recognised as C. cf. flagellaris based on a five-gene phylogenetic tree (Groenewald et al. 2013, Bakhshi et al. 2015a) cluster into at least four distinct phylogenetic clades based on the eight-gene phylogenetic tree including C. cf. gossypii, C. cf. flagellaris clades 1, 2 and 3 (Fig. 1, part 3). Three, four, six, seven, seven, seven, two and nine allele groups were distinguished for the ITS, tef1, actA, cmdA, his3, tub2, rpb2 and gapdh sequences, respectively (Table 8 ).

Cercospora cf. gossypii Lall et al., Indian Phytopath. 14: 116 (1962) ["1961"].

(Fig. 8)

Description: Leaf spots amphigenous, circular to subcircular, 1-4 mm diam, with grey-brown centre and purple-brown margins. Mycelium internal. Caespituli amphigenous, brown. Conidiophores aggregated in dense fascicles, arising from the upper cells of a well-developed, intraepidermal and substomatal, brown stroma, to $65 \mu \mathrm{m}$ diam; conidiophores pale brown to brown, simple, rarely branched, 1-4-septate, straight or flexuous caused by sympodial proliferation, almost uniform in width, often constricted at proliferating point, (35-)60-75($110) \times 4-5 \mu \mathrm{m}$. Conidiogenous cells terminal or integrated, pale brown, smooth, proliferating sympodially, $10-45 \times 3.5-5$ $\mu \mathrm{m}$, multi-local; loci thickened, darkened, refractive, apical, lateral, circumspersed, 1.5-2.5 $\mu \mathrm{m}$ diam. Conidia solitary, smooth, subcylindrical to obclavate, straight or mildly curved, successively tapering towards the apex, hyaline, 1-7-septate, apex subacute to subobtuse, base truncate to short obconically 


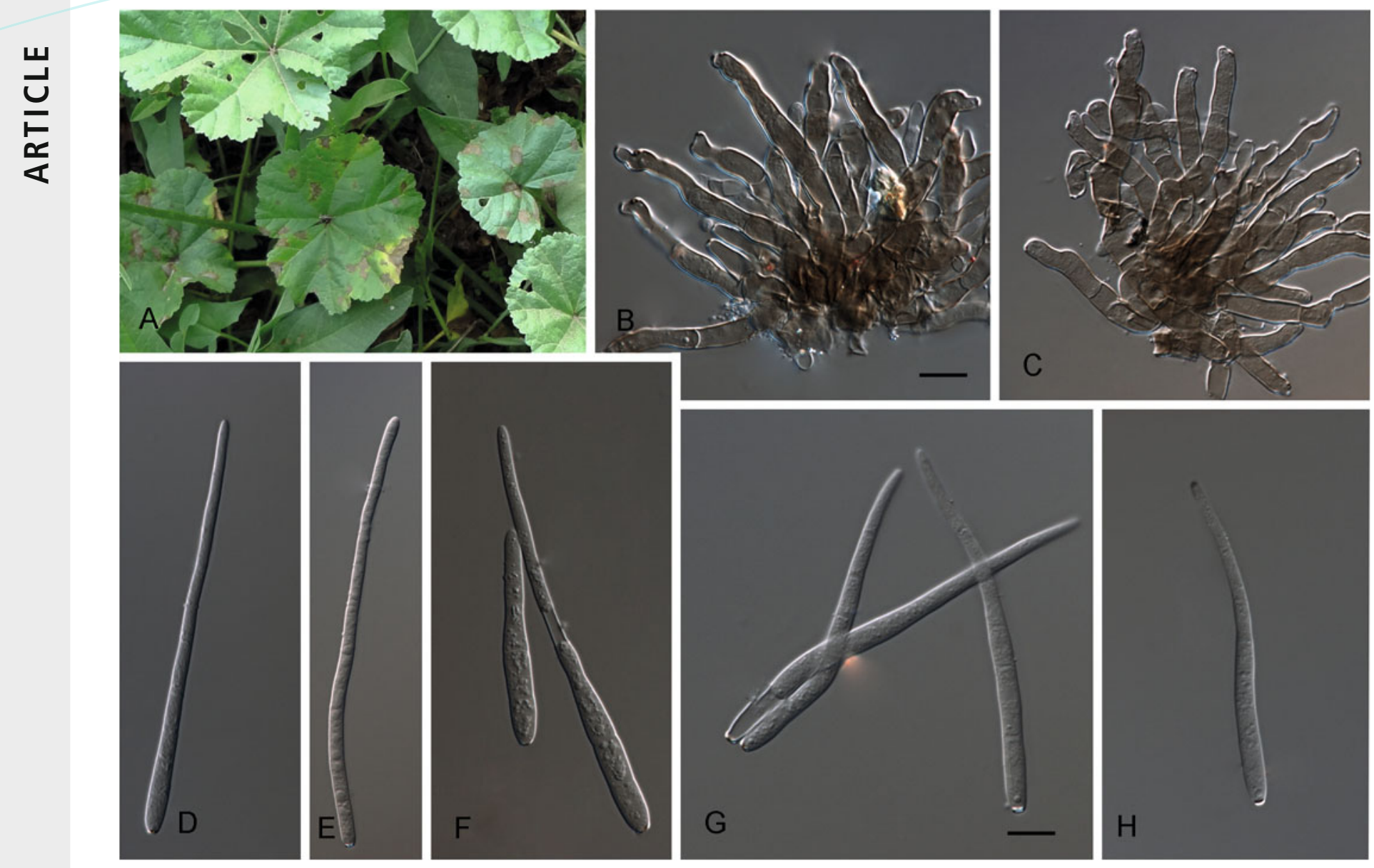

Fig. 7. Cercospora gamsiana (CPC $24909=$ CBS 144962). A. Leaf spots. B-C. Fasciculate conidiophores. D-H. Conidia. Bars $=10 \mu \mathrm{m}$.

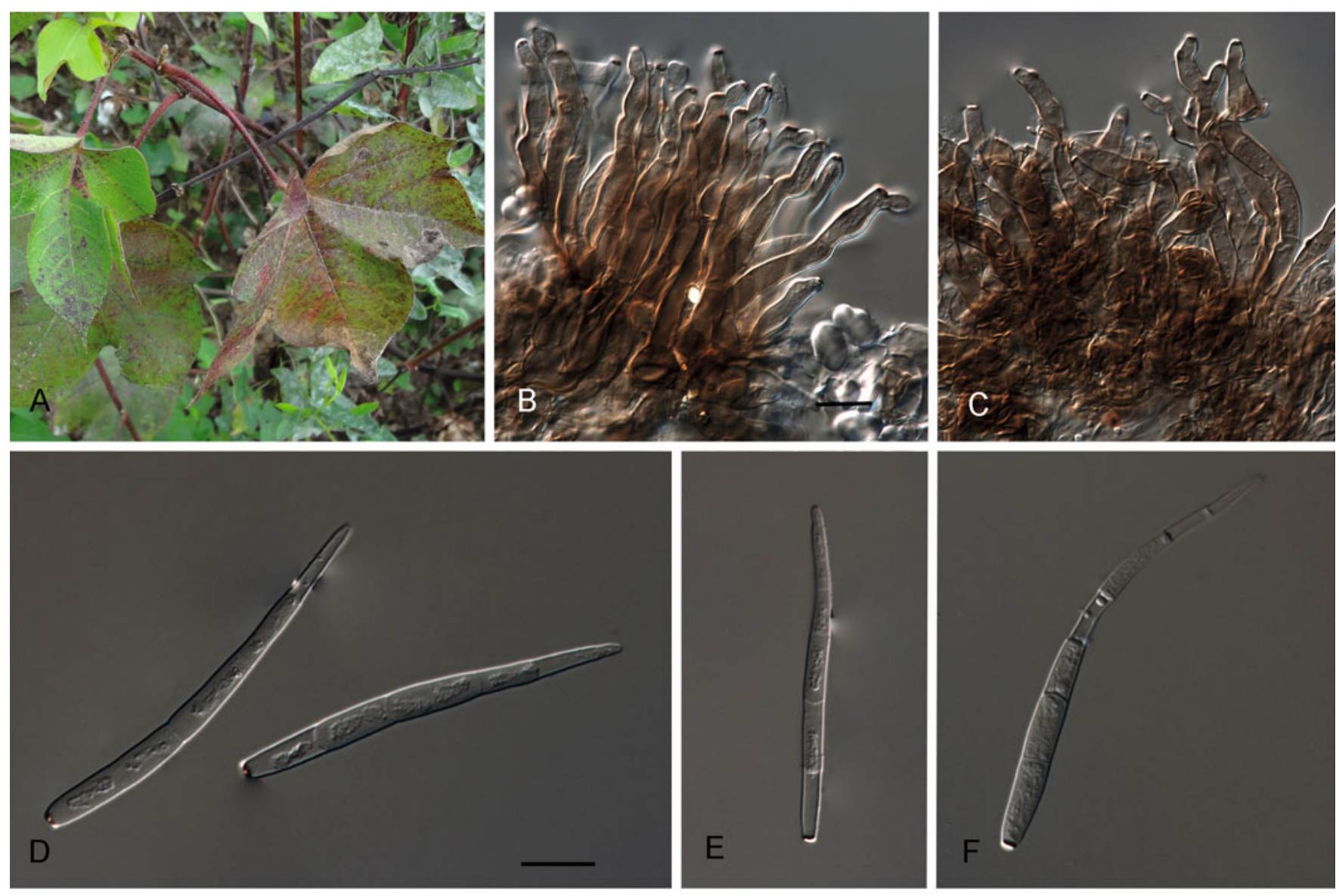

Fig. 8. Cercospora cf. gossypii (CBS 136137). A. Leaf spots. B-C. Fasciculate conidiophores. D-F. Conidia. Bars $=10 \mu \mathrm{m}$. 
Table 8. Results from allele group designation per locus for Cercospora cf. flagellaris isolates in this study. Abbreviations of loci and collection accession numbers follow Table 1.

\begin{tabular}{|c|c|c|c|c|c|c|c|c|c|c|}
\hline \multirow[t]{2}{*}{ Species } & \multirow{2}{*}{$\begin{array}{l}\text { Culture accession } \\
\text { number }\end{array}$} & \multirow[t]{2}{*}{ Host } & \multicolumn{8}{|c|}{ Allele group per locus } \\
\hline & & & ITS & tef1 & $\operatorname{act} A$ & $c m d A$ & his3 & tub2 & $r p b 2$ & gapdh \\
\hline \multirow{2}{*}{$\begin{array}{c}\text { Cercospora cf. } \\
\text { gossypii }\end{array}$} & CCTU 1055; IRAN 2650C & Hibiscus trionum & 1 & 1 & 1 & III & III & I & II & IV \\
\hline & CCTU 1070; CBS 136137 & Gossypium herbaceum & I & I & I & III & III & I & ॥ & IV \\
\hline \multirow{12}{*}{$\begin{array}{l}\text { Cercospora cf. } \\
\quad \text { flagellaris clade } 1\end{array}$} & CCTU 1007; CBS 136031 & Hydrangea sp. & II & 1 & 1 & 1 & 1 & I & II & V \\
\hline & CCTU 1027; CBS 136034 & Lepidium sativum & I & 1 & 1 & I & I & 1 & 1 & V \\
\hline & $\begin{array}{l}\text { CCTU 1031; CBS 136036; } \\
\text { IRAN 2648C }\end{array}$ & Urtica dioica & $\|$ & 1 & ॥ & I & I & I & II & V \\
\hline & CCTU 1120 & Raphanus sativus & II & 1 & 1 & 1 & I & 1 & II & V \\
\hline & $\begin{array}{l}\text { CCTU 1128; CBS 136141; } \\
\text { IRAN 2661C }\end{array}$ & Phaseolus vulgaris & ॥ & 1 & 1 & I & I & 1 & II & V \\
\hline & CCTU 1159; CBS 136148 & Arachis hypogaea & II & 1 & 1 & 1 & I & 1 & II & V \\
\hline & CCTU 1162; IRAN 2670C & Citrullus lanatus & II & 1 & 1 & I & 1 & 1 & 1 & V \\
\hline & CCTU 1168 & Phaseolus vulgaris & II & 1 & I & II & I & 1 & 1 & V \\
\hline & ССТU 1171 & Raphanus sativus & ॥ & I & I & I & I & I & ॥ & $\mathrm{V}$ \\
\hline & CPC 1051 & Populus deltoides & ॥ & I & III & ॥ & I & I & $\|$ & V \\
\hline & CBS 132653; CPC 10884 & Dysphania ambrosioides & II & 1 & 1 & III & VII & VI & II & VII \\
\hline & CPC 5441 & Amaranthus sp. & I & III & V & III & IV & III & 1 & VI \\
\hline \multirow{10}{*}{$\begin{array}{l}\text { Cercospora cf. } \\
\quad \text { flagellaris clade } 2\end{array}$} & CCTU 1059; CBS 136136 & Ecballium elaterium & II & 1 & I & I & 1 & I & I & VIII \\
\hline & CCTU 1068 & Xanthium spinosum & ॥ & IV & III & III & III & I & ॥ & VIII \\
\hline & CCTU 1085 & Xanthium strumarium & II & 1 & 1 & III & V & V & II & VIII \\
\hline & $\begin{array}{l}\text { CCTU 1115; CBS 136139; } \\
\text { IRAN 2659C }\end{array}$ & Cercis siliquastrum & ॥ & 1 & 1 & I & I & 1 & I & VIII \\
\hline & CCTU 1195 & Datura stramonium & 1 & 1 & 1 & 1 & 1 & 1 & II & IX \\
\hline & CCTU 1198; CBS 136151 & Acer velutinum & II & 1 & 1 & II & 1 & 1 & II & IX \\
\hline & CCTU 1204 & Abutilon theophrasti & 1 & I & 1 & II & I & 1 & II & IX \\
\hline & CCTU 1216 & Ecballium elaterium & II & I & 1 & 1 & 1 & 1 & II & VIII \\
\hline & $\begin{array}{l}\text { CCTU 1223; CBS 136154; } \\
\text { IRAN 2683C }\end{array}$ & Eclipta prostrata & 1 & 1 & 1 & II & 1 & 1 & II & VIII \\
\hline & CBS 132667; CPC 11643 & $\begin{array}{l}\text { Celosia argentea var. } \\
\text { cristata ( } \equiv \text { C. cristata) }\end{array}$ & III & 1 & VI & III & III & 1 & II & VIII \\
\hline \multirow{18}{*}{$\begin{array}{l}\text { Cercospora cf. } \\
\quad \text { flagellaris clade } 3\end{array}$} & CCTU 1005; IRAN 2644C & Xanthium strumarium & II & 1 & II & 1 & I & 1 & II & 1 \\
\hline & CCTU 1006; CBS 136030 & Impatiens balsamina & II & 1 & I & 1 & 1 & II & II & 1 \\
\hline & CCTU 1010; CBS 136032 & Pelargonium hortorum & II & 1 & 1 & I & 1 & I & 1 & 1 \\
\hline & CCTU 1021; CBS 136033 & Amaranthus retroflexus & II & I & 1 & I & 1 & 1 & 1 & II \\
\hline & $\begin{array}{l}\text { CCTU 1029; CBS 136035; } \\
\text { IRAN 2647C }\end{array}$ & Cucurbita maxima & 1 & I & 1 & VI & 1 & I & ॥ & II \\
\hline & CCTU 1048; CBS 136029 & Xanthium strumarium & II & I & 1 & 1 & I & I & I & 1 \\
\hline & CCTU 1064 & Amaranthus retroflexus & II & I & II & I & 1 & I & II & II \\
\hline & CCTU 1072; IRAN 2653C & Amaranthus blitoides & II & I & 1 & 1 & 1 & I & II & 1 \\
\hline & CCTU 1075 & Raphanus sativus & 1 & I & 1 & VII & 1 & II & II & 1 \\
\hline & CCTU 1084; CBS 136156 & Amaranthus sp. & II & 1 & 1 & I & 1 & II & 1 & 1 \\
\hline & $\begin{array}{l}\text { CCTU 1118; CBS 136140; } \\
\text { IRAN 2660C }\end{array}$ & Populus deltoides & II & I & ॥ & II & I & 1 & 1 & II \\
\hline & CCTU 1130; CBS 136142 & Olea europaea & II & 1 & 1 & I & 1 & I & II & I \\
\hline & CCTU 1136 & Cucurbita pepo & II & 1 & 1 & I & 1 & I & II & I \\
\hline & CCTU 1138; IRAN 2664C & Phaseolus vulgaris & I & 1 & 1 & II & 1 & 1 & II & II \\
\hline & CCTU 1139; IRAN 2665C & Phaseolus vulgaris & I & 1 & ॥ & I & I & 1 & II & I \\
\hline & $\begin{array}{l}\text { CCTU 1140; CBS 136143; } \\
\text { IRAN 2666C }\end{array}$ & Calendula officinalis & II & 1 & ॥ & I & 1 & 1 & II & II \\
\hline & CCTU 1141; CBS 136144 & Tagetes patula & I & 1 & II & I & I & VII & II & II \\
\hline & CCTU 1142; IRAN 2667C & Phaseolus vulgaris & II & II & III & IV & II & 1 & I & 1 \\
\hline
\end{tabular}


Table 8. (Continued).

\begin{tabular}{|c|c|c|c|c|c|c|c|c|c|c|}
\hline \multirow[t]{2}{*}{ Species } & \multirow{2}{*}{$\begin{array}{l}\text { Culture accession } \\
\text { number }\end{array}$} & \multirow[t]{2}{*}{ Host } & \multicolumn{8}{|c|}{ Allele group per locus } \\
\hline & & & ITS & tef1 & $\operatorname{act} A$ & $c m d A$ & his3 & tub2 & rpb2 & gapdh \\
\hline & CCTU 1143; CBS 136145 & Datura stramonium & II & I & 1 & 1 & I & 1 & 1 & 1 \\
\hline & CCTU 1147 & Urtica dioica & I & I & I & II & I & 1 & I & II \\
\hline & CCTU 1150 & Buxus microphylla & II & I & I & II & 1 & 1 & II & II \\
\hline & CCTU 1154; CBS 136147 & Abutilon theophrasti & II & I & I & I & I & I & I & II \\
\hline & CCTU 1155.11 & Phaseolus vulgaris & II & I & I & I & I & I & I & I \\
\hline & CCTU 1156 & Xanthium strumarium & II & I & II & II & I & I & I & II \\
\hline & CCTU 1158; IRAN 2668C & Xanthium strumarium & II & I & I & I & I & I & I & I \\
\hline & CCTU 1160; CBS 136149 & Vicia faba & II & I & 1 & I & I & I & II & I \\
\hline & CCTU 1161; IRAN 2669C & Phaseolus vulgaris & II & I & 1 & I & I & I & II & I \\
\hline & CCTU 1167; CBS 136150 & Anubias sp. & II & I & II & I & I & I & I & I \\
\hline & CCTU 1175; IRAN 2673C & Phaseolus vulgaris & II & I & 1 & II & I & 1 & I & I \\
\hline & CCTU 1209; CBS 136152 & Glycine max & II & I & II & I & I & I & II & 1 \\
\hline & CCTU 1210; IRAN 2679C & Glycine max & II & I & II & 1 & 1 & I & II & II \\
\hline & CCTU 1211 & Glycine max & II & I & I & I & 1 & I & I & I \\
\hline & $\begin{array}{l}\text { CCTU 1212; CBS 136153; } \\
\text { IRAN 2680C }\end{array}$ & Silybum marianum & II & I & 1 & 1 & 1 & 1 & II & II \\
\hline & CCTU 1218; IRAN 2682C & Hibiscus trionum & I & I & I & I & I & I & I & I \\
\hline & CBS 115482; CPC 4410 & Citrus sp. & II & I & IV & III & VI & IV & I & III \\
\hline & CBS 143.51; CPC 5055 & Bromus sp. & II & 1 & 1 & I & I & IV & II & I \\
\hline
\end{tabular}

truncate, $(30-) 65-90(-160) \times 2-4 \mu \mathrm{m}$; hila distinctly thickened, darkened, refractive, 1-2 $\mu \mathrm{m}$ diam.

Notes: This clade includes two isolates obtained from $G$. herbaceum and Hib. trionum, both in the Malvaceae (Fig. 1, part 3). Cercospora althaeina, C. fagopyri, C. malayensis (C. apii s. lat.), C. gossypii, C. gossypiicola, C. gossypina and C. Ihuillieri (C. apii s. lat.) are six Cercospora species which have been reported until now on Gossypium and Hibiscus host genera (Crous \& Braun 2003, https://nt.ars-grin.gov/ fungaldatabases/). This species is phylogenetically distinct from C. althaeina (Fig. 1) and C. fagopyri (Groenewald et al. 2013, Bakhshi et al. 2015a). Cercospora gossypina is distinguished from this species in that it induces wider leaf spots $(0.5-10 \mathrm{~mm})$, and has unbranched, longer and wider conidiophores (75-250 × 4-6.5 $\mu \mathrm{m})$ (Hsieh \& Goh 1990). Cercospora malayensis is distinguished from C. cf. gossypii in that it has elliptical, yellow to tan leaf spots; unbranched, 1-8-septate conidiophores and mostly terminal conidiogenous cells and somewhat longer conidia (50-270 × 2.5-4 $\mu \mathrm{m})$ (Little 1987). Cercospora gossypiicola (Narayan et al. 2001) and C. Ihuillieri (Montegut 1967) resemble C. apii (with acicular conidia), but are different. They do not have stromata, and form less conidiophores per fascicle. The description of $C$. gossypii (Lall et al. 1961) is rather close to this taxon. The type of $C$. gossypii is from India. Thus, fresh material is needed from India to resolve the application of the name C. gossypii.

Specimens examined: Iran: Ardabil Province: Moghan, on Gossypium herbaceum (Malvaceae), Oct. 2011, M. Bakhshi (IRAN 17073F, CCTU 1070 = CBS 136137); Moghan, on Hibiscus trionum
(Malvaceae), Oct. 2011, M. Bakhshi (IRAN 17074F, CCTU $1055=$ IRAN 2650C).

Cercospora cf. flagellaris Ellis \& G. Martin, Am. Nat. 16: 1003 (1882).

\section{Clade 1; Clade 2; Clade 3}

In view of the overlap between the morphological characters of these three clades, we provide a single over-arching description here.

Description: Mycelium internal. Caespituli amphigenous, brown. Conidiophores aggregated in loose to dense fascicles, arising from a weakly to well-developed, intraepidermal and substomatal, brown stroma; conidiophores pale brown to brown, 2-18-septate, straight, sinuous to distinctly geniculate, flexuous, simple, unbranched or rarely branched, uniform or irregular in width, sometimes constricted at septa and proliferating point, (75-)130-165(-300) × 4-5.5 $\mu \mathrm{m}$ in clade 1 ; (30-)80$120(-210) \times 3.5-5.5 \mu \mathrm{m}$ in clade $2 ;(25-) 60-95(-230) \times 3.5-$ $5.5 \mu \mathrm{m}$ in clade 3 . Conidiogenous cells integrated, terminal, proliferating sympodially, mono- or multi-local; loci thickened, darkened, apical, lateral or circumspersed, 1.5-2.5 $\mu \mathrm{m}$ diam. Conidia solitary, hyaline, subcylindrical, filiform to obclavate, straight to slightly curved, with truncate to obconically truncate base and subacute to subobtuse apices, (60-)125-170(-300) $\times 3-5 \mu \mathrm{m}, 5-20$-septate in clade 1 ; (25-)60-95(-260) × 2.5$4.5 \mu \mathrm{m},(2-) 8-11(-25)$-septate in clade 2; (30-)100-155(-320) $\times 2-5 \mu \mathrm{m},(2-) 10-14(-28)$-septate in clade 3 ; hila distinctly thickened, darkened, refractive, 1-2 $\mu \mathrm{m}$ diam. 
Notes: Screening the remaining isolates of $C$. cf. flagellaris, with three more genomic loci in this study (tub2, rpb2 and gapdh), clusters them into at least three distinct clades in the eight-gene phylogenetic tree (Fig. 1, part 3); clade 1 is sister to C. cf. gossypii; clade 3 is sister to C. convolvulicola and clade 2 is sister to the clade including $C$. cf. flagellaris clade 3 and $C$. convolvulicola. However, there is a high level of variation in morphological characteristics between different isolates of these three clades. In addition, several isolates originating from diverse hosts and families reside in these three clades and there is also overlap between host ranges among them. Different names can therefore be applied to these clades, and therefore we prefer to simply regard them as distinct phylogenetic species for now. To resolve their taxonomy, fresh collections authentic for the names (based on host and country) need to be recollected and included in future studies.

\section{Cercospora cf. flagellaris Clade 1}

Specimens examined: Fiji: on Amaranthus sp. (Amaranthaceae), C.F. Hill (CPC 5441). - Iran: Guilan Province: Talesh, Khotbeh Sara, on leaves of Phaseolus vulgaris (Fabaceae), Oct. 2012, M. Bakhshi (CCTU 1128 = IRAN 2661C = CBS 136141); Talesh, Jamakuh, on leaves of Raphanus sativus (Brassicaceae), Nov. 2011, M. Bakhshi (IRAN 17042F, CCTU 1120); Talesh, Dulbin, on Hydrangea sp. (Hydrangeaceae), Jul. 2011, M. Bakhshi (IRAN 17039F, CCTU 1007 = CBS 136031). Guilan Province: Kiashahr, on leaves of Ph. vulgaris, Aug. 2012, M. Bakhshi (CCTU 1168 = IRAN 2715C); Kiashahr, on leaves of $R$. sativus, Aug. 2012, M. Bakhshi (IRAN 17041F, CCTU 1171); Kiashahr, on leaves of Arachis hypogea (Fabaceae), Aug. 2012, M. Bakhshi (CCTU 1159 = CBS 136148); Sowme`eh Sara, Dowgur, on leaves of Urtica dioica (Urticaceae), Jun. 2012, M. Bakhshi (IRAN 17043F, CCTU $1031=$ IRAN 2648C = CBS 136036); Chamkhaleh, on leaves of Lepidium sativum (Brassicaceae), Jun. 2012, M. Bakhshi (IRAN 17040F, CCTU 1027 = CBS 136034); Lahijan, Rudboneh, on leaves of Citrullus lanatus (Cucurbitaceae), Aug. 2012, M. Bakhshi (IRAN 17038F, CCTU $1162=$ IRAN 2670C). South Africa: Limpopo Province: Messina, 30 Apr. 1995, on Populus deltoides (Salicaceae), P.W. Crous (CPC 1051). - South Korea: Jeju, on Dysphania ambrosioides (syn. Chenopodium ambrosioides) (Chenopodiaceae), 12 Nov. 2003, H.D. Shin (CBS $132653=$ CPC 10884) (as C. chenopodii-ambrosioidis).

\section{Cercospora cf. flagellaris Clade 2}

Specimens examined: Iran: Ardabil Province: Moghan, on leaves of Xanthium spinosum (Astraceae), Oct. 2011, M. Bakhshi (IRAN 17049F, CCTU 1068); Moghan, on leaves of Xanthium strumarium (Asteraceae), Oct. 2011, M. Bakhshi (IRAN 17050F, CCTU 1085); Moghan, on leaves of Ecballium elaterium (Cucurbitaceae), Oct. 2011, M. Bakhshi (IRAN 17047F, CCTU 1059 = CBS 136136); Moghan, on leaves of E. elaterium, Oct. 2012, M. Bakhshi (IRAN 17048F, CCTU $1216=$ IRAN 2717C); Moghan, on leaves of Abutilon theophrasti (Malvaceae), Oct. 2012, M. Bakhshi (IRAN 17044F, CCTU 1204). Guilan Province: Astara, on leaves of Cercis siliquastrum (Caesalpinaceae), Oct. 2012, M. Bakhshi (CCTU 1115 = IRAN 2659C = CBS 136139); Talesh, Khotbeh Sara, on leaves of Eclipta prostrata (Astraceae), Oct. 2012, M. Bakhshi (CCTU $1223=$ IRAN 2683C = CBS 136154); Talesh, on leaves of Datura stramonium (Solanaceae), Oct. 2012, M. Bakhshi (IRAN 17046F, CCTU 1195). Mazandaran Province: Ramsar, on leaves of Acer velutinum (Aceraceae), Sep. 2012, M. Bakhshi (IRAN 17045F, CCTU 1198 = CBS 136151). - South Korea: Hoengseong, on Celosia argentea var. cristata (syn. C. cristata) (Amaranthaceae), 11 Oct. 2004, H.D. Shin (CBS 132667 = CPC 11643).

\section{Cercospora cf. flagellaris Clade 3}

Specimens examined: Iran: Guilan Province: Rudsar, on leaves of Cucurbita maxima (Cucurbitaceae), Oct. 2012, M. Bakhshi (CCTU 1029 = IRAN 2647C = CBS 136035); Rudsar, Korjehposht, on leaves of Tagetes patula (Asteraceae), Aug. 2012, M. Bakhshi (IRAN 17065F, CCTU 1141 = CBS 136144); Talesh, Khotbeh Sara, on leaves of Cucurbita pepo (Cucurbitaceae), Jun. 2012, M. Bakhshi (CCTU 1136); Khotbeh Sara, on leaves of Vicia faba (Fabaceae), Oct. 2012, M. Bakhshi (IRAN 17067F, CCTU 1160 = CBS 136149); Khotbeh Sara, on leaves of Calendula officinalis (Asteraceae), Jun. 2012, M. Bakhshi (IRAN 17058F, CCTU $1140=$ IRAN 2666C = CBS 136143); Talesh, Khalif Abad, on Ph. vulgaris, Jul. 2012, M. Bakhshi (CCTU 1142 = IRAN 2667C); Talesh, Dulbin, on leaves of X. strumarium, Jul. 2011, M. Bakhshi (IRAN 17069F, CCTU 1005 = IRAN 2644C); Dulbin, on leaves of Impatiens balsamina (Balsaminaceae), Jul. 2011, M. Bakhshi (IRAN 17062F, CCTU 1006 = CBS 136030); Dulbin, on leaves of Pelargonium hortorum (Geraniaceae), Aug. 2011, M. Bakhshi (CCTU 1010 = CBS 136032); Talesh, Jowkandan, on leaves of Po. deltoides, Oct. 2012, M. Bakhshi (CCTU 1118 = IRAN 2660C = CBS 136140); Talesh, Jowkandan, on leaves of Oenothera biennis (Onagraceae), Oct. 2012, M. Bakhshi (IRAN 17051F, CCTU 1172); Talesh, on leaves of $D$. stramonium, Oct. 2012, M. Bakhshi (IRAN 17059F, CCTU 1143 = CBS 136145); Guilan Province: Astara, Chubar, on leaves of Ph. vulgaris, Jun. 2012, M. Bakhshi (CCTU $1138=$ IRAN 2664C, CCTU $1139=$ IRAN 2665C); Rasht, Khomam, on leaves of $X$. strumarium, Aug. 2012, M. Bakhshi (IRAN 17068F, CCTU 1156); Khomam, on leaves of $A b$. theophrasti, Aug. 2012, M. Bakhshi (IRAN 17052F, CCTU $1154=$ CBS 136147); Langarud, Otaqvar, on leaves of $X$. strumarium, Aug. 2012, M. Bakhshi (CCTU 1158 = IRAN 2668C); Lahijan, Rudboneh, on leaves of Ph. vulgaris, Aug. 2012, M. Bakhshi (CCTU $1161=$ IRAN 2669C); Guilan Province: Fuman, on leaves of $P h$. vulgaris, Aug. 2012, M. Bakhshi (CCTU 1155.11); Fuman, on leaves of Buxus microphylla (Buxaceae), Jul. 2012, M. Bakhshi (IRAN 17057F, CCTU 1150); Fuman, on leaves of Amaranthus retroflexus, Sep. 2011, $M$. Bakhshi (IRAN 17054F, CCTU 1021 = CBS 136033); Sowme'eh Sara, Dowgur, on leaves of Ph. vulgaris, Aug. 2012, M. Bakhshi (CCTU 1175 = IRAN 2673C); Sowme`eh Sara, Bahambar, on leaves of $R$. sativus, Aug. 2012, M. Bakhshi (IRAN 17063F, CCTU 1075); Kiashahr, on leaves of Anubias sp. (Araceae), Oct. 2012, M. Bakhshi (IRAN 17056F, CCTU 1167 = CBS 136150); Masal, on leaves of $U$. dioica, Aug. 2012, M. Bakhshi (IRAN 17066F, CCTU 1147). Zanjan Province: Tarom, Pasar, on leaves of $X$. strumarium, Sep. 2011, M. Bakhshi (IRAN 17070F, CCTU 1048 = CBS 136029); Tarom, on leaves of Olea europaea (Oleaceae), Nov. 2011, M. Torbati $($ CCTU $1130=$ CBS 136142). Ardabil Province: Moghan, on leaves of Silybum marianum (Astraceae), Oct. 2012, M. Bakhshi (IRAN 17064F, CCTU 1212 = IRAN 2680C = CBS 136153); Moghan, on leaves of $A$. retroflexus, Oct. 2011, M. Bakhshi (IRAN 17053F, CCTU 1064); Moghan, on leaves of Amaranthus sp., Oct. 2011, M. Bakhshi (IRAN 17055F, CCTU 1084 = CBS 136156); Moghan, on leaves of Amaranthus blitoides, Oct. 2011, M. Bakhshi (CCTU $1072=$ IRAN 
2653C); Moghan, on leaves of Glycine max (Fabaceae), Oct. 2012, M. Bakhshi (IRAN 17060F, CCTU 1209 = CBS 136152, CCTU 1210 = IRAN 2679C, CCTU 1211); Moghan, on leaves of Hib. trionum, Oct. 2012, M. Bakhshi (IRAN 17061F, CCTU 1218 = IRAN 2682C). South Africa: Limpopo Province: Messina, on Citrus sp. (Rutaceae), M.C. Pretorius (CBS $115482=\mathrm{CPC} 4410)$. Unknown, on Bromus sp. (Poaceae), M.D. Whitehead (CBS 143.51 = CPC 5055).

\section{Cercospora sp. G complex}

The 16 isolates previously recognised as Cercospora sp. G based on a five-gene phylogenetic tree (Groenewald et al. 2013, Bakhshi et al. 2015a) cluster into two distinct phylogenetic clades based on the eight-gene phylogenetic tree (Fig. 1, part 1). One, four, one, two, two, two, three and two allele groups were detected for the ITS, tef1, actA, cmdA, his3, tub2, rpb2 and gapdh sequences, respectively (Table 9 ).

\section{Cercospora sp. G Clade 1}

Description: Mycelium internal. Caespituliamphigenous, brown. Conidiophores aggregated in loose fascicles, arising from a moderately developed, intraepidermal and substomatal, brown stroma, to $35 \mu \mathrm{m}$ diam; conidiophores pale brown to brown, 2-11-septate, straight to flexuous, simple, unbranched, uniform in width, (55-)110-150(-260) × 3.5-5 $\mu \mathrm{m}$. Conidiogenous cells integrated, terminal, proliferating sympodially, mono- and multilocal; loci thickened, darkened, apical or formed on shoulders caused by sympodial proliferation, 1.5-2.5 $\mu \mathrm{m}$ diam. Conidia solitary, hyaline, subcylindrical, filiform to obclavate, straight to slightly curved, with truncate to obconically truncate base and subacute to subobtuse apices, (40-)75-100(-165) $\times 2-4 \mu \mathrm{m}$, 4-15-septate; hila distinctly thickened, darkened, refractive, 1-2 $\mu \mathrm{m}$ diam.
Specimens examined: Iran: Guilan Province: Talesh, Dulbin, on leaves of Plantago major, Jul. 2011, M. Bakhshi (IRAN 17085F, CCTU 1015 = IRAN 2645C = CBS 136024); Talesh, Kishonben, on leaves of Bidens tripartita (Asteraceae), Sept. 2012, M. Bakhshi (IRAN 17084F, CCTU 1197). - New Zealand: Manurewa, on Salvia viscosa (Lamiaceae), C.F. Hill (CPC 5438) (as C. salviicola).

\section{Cercospora sp. G Clade 2}

Description: Mycelium internal. Caespituli amphigenous, brown. Conidiophores aggregated in loose to dense fascicles, arising from a weakly to well-developed, intraepidermal and substomatal, brown stroma, to $50 \mu \mathrm{m}$ diam; conidiophores pale brown to brown, 3-11-septate, straight to flexuous, simple, unbranched, uniform in width, (30-)65-105(-240) × 2.5-5 $\mu \mathrm{m}$. Conidiogenous cells integrated, terminal, proliferating sympodially, $10-30 \times 2.5-5 \mu \mathrm{m}$, mono- or multi-local; loci distinctly thickened, darkened and somewhat refractive, apical or formed on shoulders caused by sympodial proliferation, 1.5-2.5 $\mu \mathrm{m}$ diam. Conidia solitary, subcylindrical, filiform to obclavate, straight to slightly curved, hyaline, (25-)75-110($200) \times 3.5-5.5 \mu \mathrm{m},(3-) 8-15(-20)$-septate, with subacute to subobtuse apices and truncate to obconically truncate bases; hila thickened, darkened, refractive, 1-2 $\mu \mathrm{m}$ diam.

Notes: Isolates of Cercospora sp. G clustered in two distinct clades with high posterior probability in the eight-gene phylogenetic tree (Fig. 1, part 1). However, several isolates from diverse host families cluster in these two clades, to which different names can be applied. Moreover, there is also overlap between host ranges of the two clades. On the other hand, there is no morphological basis to divide them into two distinct species. Based on the gene loci screened in the present study, we were unable to resolve the taxonomy of

Table 9. Results from allele group designation per locus for Cercospora sp. G isolates in this study. Abbreviations of loci and collection accession numbers follow Table 1.

\begin{tabular}{|c|c|c|c|c|c|c|c|c|c|c|}
\hline \multirow[t]{2}{*}{ Species } & \multirow[t]{2}{*}{ Culture accession number } & \multirow[t]{2}{*}{ Host } & \multicolumn{8}{|c|}{ Allele group per locus } \\
\hline & & & ITS & tef1 & actA & cmdA & his3 & tub2 & $r p b 2$ & gapdh \\
\hline \multirow[t]{3}{*}{$\begin{array}{l}\text { Cercospora sp. G } \\
\quad \text { clade } 1\end{array}$} & $\begin{array}{l}\text { CCTU 1015; CBS 136024; } \\
\text { IRAN 2645C }\end{array}$ & Plantago major & 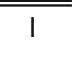 & IV & 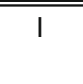 & 1 & 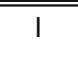 & 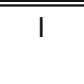 & בIII & III \\
\hline & CCTU 1197 & Bidens tripartita & 1 & IV & 1 & 1 & 1 & 1 & I & II \\
\hline & CPC 5438 & Salvia viscosa & I & II & 1 & 1 & II & II & _- & II \\
\hline \multirow{13}{*}{$\begin{array}{l}\text { Cercospora sp. G } \\
\quad \text { clade } 2\end{array}$} & CCTU 1002 & Celosia cristata & I & 1 & I & I & I & I & I & I \\
\hline & CCTU 1020; CBS 136023 & Sorghum halepense & 1 & 1 & 1 & I & 1 & 1 & II & I \\
\hline & CCTU 1030; CBS 136026 & Bidens tripartita & I & III & I & I & II & I & I & I \\
\hline & CCTU 1046 & Plantago major & I & 1 & 1 & I & 1 & 1 & 1 & 1 \\
\hline & CCTU 1053; CBS 136027 & Cichorium intybus & I & III & I & I & II & I & I & I \\
\hline & CCTU 1054 & Amaranthus sp. & 1 & 1 & 1 & I & I & I & I & I \\
\hline & CCTU 1058 & Helminthotheca echioides & 1 & 1 & 1 & 1 & 1 & I & I & 1 \\
\hline & CCTU 1079; CBS 136025 & Amaranthus retroflexus & 1 & 1 & 1 & I & 1 & I & I & 1 \\
\hline & CCTU 1090 & Abutilon theophrasti & I & 1 & I & I & I & I & I & I \\
\hline & CCTU 1116 & Plantago major & I & 1 & 1 & 1 & I & I & I & I \\
\hline & CCTU 1122 & Amaranthus sp. & 1 & I & I & I & I & I & I & I \\
\hline & CCTU 1144; CBS 136130 & Cucurbita maxima & I & I & I & ॥ & I & I & I & I \\
\hline & CBS 115518; CPC 5360 & Bidens frondosa & I & IV & I & I & I & I & _- & I \\
\hline
\end{tabular}


these isolates and for now prefer to treat them as unresolved phylogenetic species. As with $C$. cf. flagellaris, in order to resolve their taxonomy, fresh collections from the same host and country as the original material need to be recollected and included in future studies.

Specimens examined: Iran: Zanjan Province: Tarom, Pasar, on leaves of P. major, Oct. 2011, M. Bakhshi (CCTU 1046); Tarom, Pasar, on leaves of $P$. major, Nov. 2011, M. Bakhshi (IRAN 17093F, CCTU 1116). Guilan Province: Talesh, Kishonben, on leaves of Bi. tripartita, Oct. 2012, M. Bakhshi (IRAN 17091F, CCTU 1030 = CBS 136026); Talesh, on leaves of Sorghum halepense (Poaceae), Sep. 2011, M. Bakhshi (IRAN 17094F, CCTU 1020 = CBS 136023); Talesh, Dolbin, on leaves of Celosia cristata, Jul. 2011, M. Bakhshi (IRAN 17092F, CCTU 1002). Ardabil Province: Moghan, on leaves of $A$. retroflexus, Oct. 2011, M. Bakhshi (IRAN 17088F, CCTU 1079 = CBS 136025); Moghan, on leaves of Amaranthus sp., Oct. 2011, M. Bakhshi (IRAN 17089F, CCTU 1054); Moghan, on leaves of Helminthotheca echioides (Asteraceae), Oct. 2011, M. Bakhshi (IRAN 17086F, CCTU 1058); Moghan, on leaves of Ab. theophrasti, Oct. 2012, M. Bakhshi (IRAN 17087F, CCTU 1090). Guilan Province: Talesh, Jamakuh, on leaves of Amaranthus sp., Nov. 2011, M. Bakhshi (IRAN 17090F, CCTU 1122); Masal, on leaves of Cu. maxima, Jul. 2012, M. Bakhshi (CCTU 1144 = CBS 136130); Sowme`eh Sara, Dowgur, on leaves of Cichorium intybus (Asteraceae), Jun. 2012, M. Bakhshi (CCTU 1053 = CBS 136027). - New Zealand: Kopuku, on Bidens frondosa (Asteraceae), C.F. Hill (CBS $115518=$ CPC 5360).

\section{Identification of the best-performing DNA barcode}

\section{Kimura-2-parameter values}

The Kimura-2-parameter distribution graphs (Fig. 9) visualise the inter- and intraspecific distances per locus corresponding to the barcoding gap (Hebert et al. 2003, Schoch et al. 2012). A useful barcoding locus should have no overlap between the inter- and intraspecific K2P distances and generally should have an average interspecific distance that is at least ten times as high as the average intraspecific distance of that locus (Quaedvlieg et al. 2012, Verkley et al. 2013, Stielow et al. 2015).

The eight tested loci showed varying degrees of overlap in their K2P distribution between inter- and intraspecific variation graphs (Fig. 9). In this dataset, the average interspecific variation in ITS dataset was very low (0.002) compared to its intraspecific variation (0.0005), leading to a very low interto intraspecific variation ratios of $4: 1$ for this locus (Fig. 9, Table 4). This low ratio is far below the recommended 10:1 ratio, indicating a general lack of natural variation within the ITS locus, making it ill-suited for effective identification of the individual species of Cercospora. Due to the presence of introns in the seven protein coding loci, these genes provide much higher interspecific variation than the more conserved ITS locus. These protein coding genes had K2P inter- to intraspecific variation ratios of 127:1 for tef1, 76:1 for $c m d A$, 74:1 for rpb2, 71:1 for tub2, 44:1 for gapdh, 15:1 for act $A$ and 13:1 for his3 (Table 4), making them all suitable for reliable species resolution of Cercospora spp. As the tef1, cmdA, rpb2, tub2 and gapdh have the largest barcoding gap, these loci should give the highest species resolution. However, all of these genes do have overlap between the inter- and intraspecific K2P distances (as is evident in the graphs of Fig. 9 ), suggesting that no one of them can serve as a single ideal barcoding locus for Cercospora spp.

Molecular phylogenetic resolution (clade recovery) Based on the results of the individual gene tree assessments, no single gene region was found which could reliably distinguish all species, and occurrences of the same sequence(s) shared between multiple species were observed in each locus.

The ITS phylogeny had low resolution and was only able to distinguish C. chenopodii, C. solani and C. sorghicola from the other included species. The remaining loci had different levels of resolution. The gapdh region was more effective and could resolve $61 \%$ of 28 lineages, whereas his3, tub2, act $A$, tef1, cmd $A$ and $r p b 2$ had respectively $48,43,43,39,32$ and $32 \%$ clade recovery. Based on the gapdh region, we were able to distinguish 17 of the 28 species clades, including $C$. althaeina, C. armoraciae, C. bizzozeriana, C. chenopodii, C. conyzae-canadensis, C. cf. flagellaris clade 1, C. cf. flagellaris clade 2, C. cf. gossypii, C. pseudochenopodii, C. cf. richardiicola, C. rumicis, C. solani, C. sorghicola, Cercospora sp. G clade 1, Cercospora sp. G clade 2, C. violae and C. cf. zinnia; whereas, 13 species clades including C. althaeina, C. chenopodii, C. conyzae-canadensis, C. cylindracea, C. pseudochenopodii, C. cf. richardiicola, C. rumicis, C. solani, C. sorghicola, C. uwebrauniana, C. violae, C. zebrina and C. cf. zinniae were distinguished in the his 3 phylogeny; 12 species clades including C. althaeina, C. chenopodii, C. conyzaecanadensis, C. cylindracea, C. iranica, C. pseudochenopodii, C. cf. richardiicola, C. solani, C. sorghicola, Cercospora sp. T, C. uwebrauniana and C. cf. zinniae were distinguished in the tub2 phylogeny; 12 species clades including $C$. althaeina, C. chenopodii, C. convolvulicola, C. conyzae-canadensis, C. cylindracea, C. pseudochenopodii, C. cf. richardiicola, C. solani, C. sorghicola, C. violae, C. zebrina and C. cf. zinniae were distinguished in the actA phylogeny; 11 species clades including C. bizzozeriana, C. chenopodii, C. conyzae-canadensis, C. pseudochenopodii, C. cf. richardiicola, C. rumicis, C. solani, C. sorghicola, C. uwebrauniana, C. violae and C. cf. zinniae were distinguished in the tef1 phylogeny; nine species clades including C. convolvulicola, C. conyzae-canadensis, C. iranica, C. cf. richardiicola, C. solani, C. sorghicola, Cercospora sp. T, C. violae and $C$. cf. zinniae were distinguished in the $\mathrm{cmd} A$ phylogeny; and nine species clades including C. bizzozeriana, C. chenopodii, C. conyzae-canadensis, C. pseudochenopodii, C. cf. richardiicola, C. solani, C. sorghicola, C. zebrina and C. cf. zinniae were distinguished in the rpb2 phylogeny.

Therefore, the gapdh phylogeny displayed a high resolution and had the highest clade recovery and was responsible for resolving most of the cryptic taxa within $C$. apii, C. armoraciae, C. beticola, Cercospora sp. G, and C. cf. flagellaris.

\section{DISCUSSION}

In this study, we re-assessed species of the genus Cercospora using a combined approach based on the evaluation of an 

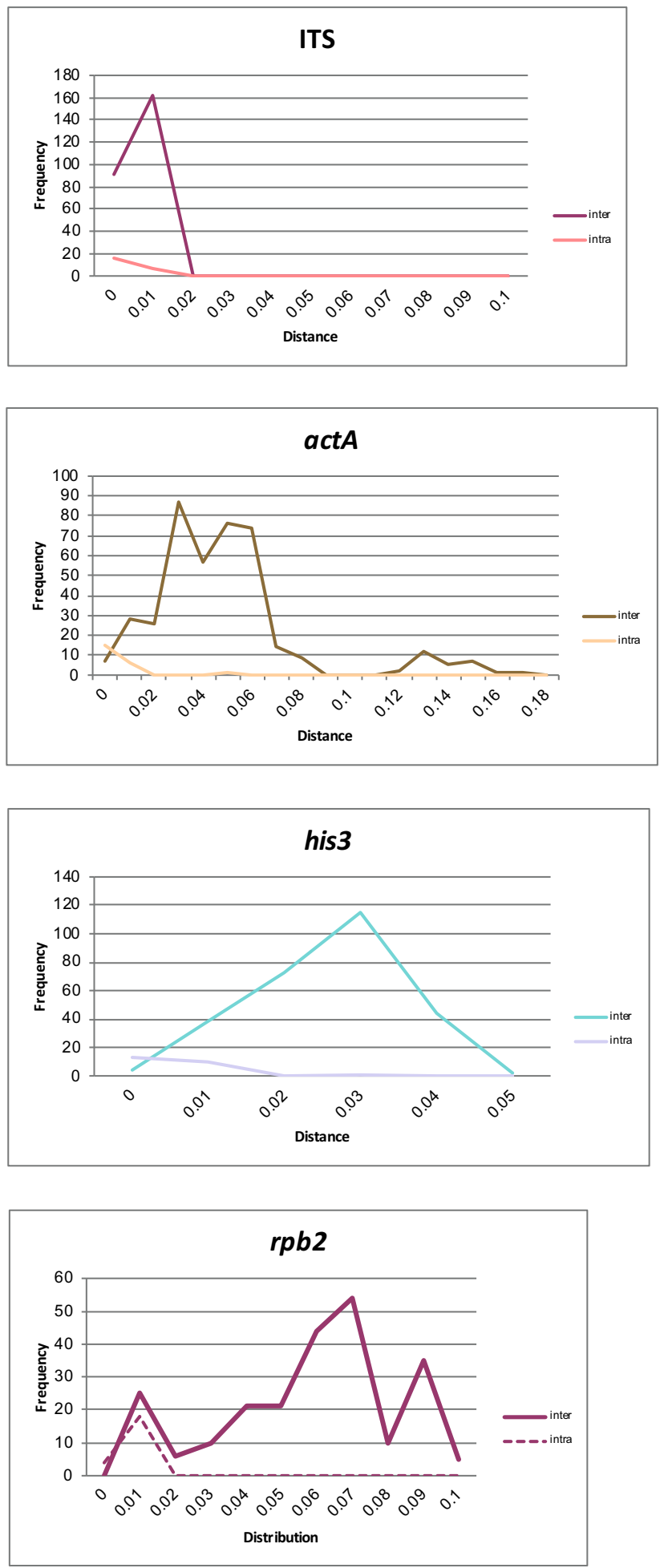
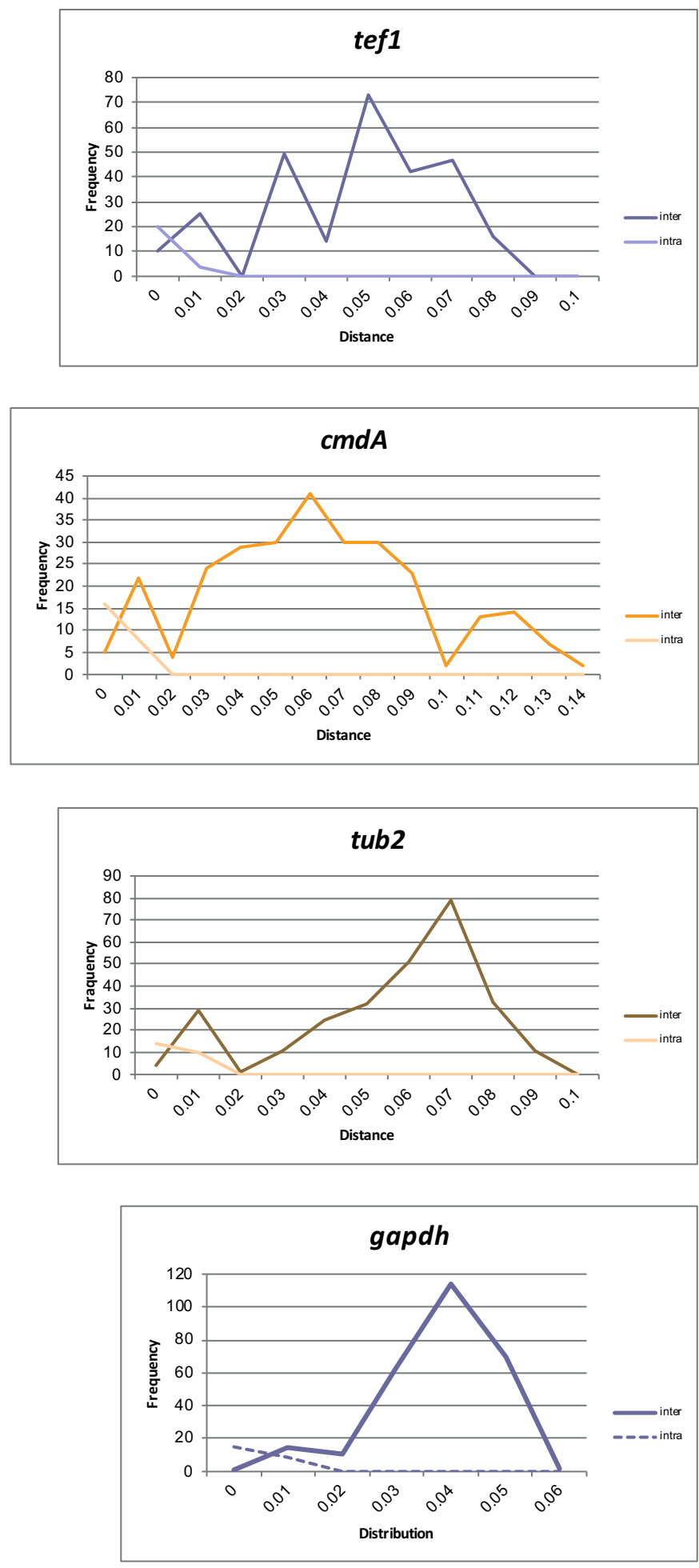

Fig. 9. Frequency distributions of the Kimura-2-parameter distance (barcoding gap) for the eight loci.

eight-gene molecular DNA sequence dataset, host, and morphological data (in those cases where morphological variation was present). In recent years, the rapid advance of molecular techniques has brought about the possibility of a more precise species delimitation and a better consideration of the evolution of fungi. It is well-known that many fungal taxa based on morphology or on sequence data of the commonly used fungal barcode ITS region of the nrDNA operon (Schoch et al. 2012) hide cryptic species complexes when molecular data from multiple gene regions are considered (Lombard et al. 2010, Cabral et al. 2012, Crous et al. 2013, Groenewald et al. 2013, Quaedvlieg et al. 2013, Woudenberg et al. 2013). This is most likely an underestimation for many fungal taxa. For instance, the Colletotrichum acutatum species complex, once considered to be a single species, has been shown to include at least 31 cryptic taxa (Damm et al. 2012). In the present study, phylogenetic inference also revealed cryptic species complexes that could not be distinguished based on geography, host association, morphology, or ITS sequence data alone. 
Before this study, Groenewald et al. (2013) and Bakhshi et al. (2015a) inferred phylogenies of Cercospora based on sequence data of five genomic loci (ITS, tef1, act $A, c m d A$ and his3). Their results showed the importance of all five loci in a combined analysis for Cercospora taxonomy (Groenewald et al. 2013, Bakhshi et al. 2015a). Despite this, the sequences of these five loci were too conserved in Cercospora, and it was not possible to identify a single gene as the best DNA barcoding locus. In addition, several species complexes remained unresolved (Groenewald et al. 2013, Bakhshi et al. 2015a). To overcome these deficiencies, three more potential candidate gene regions, tub2, rpb2, and gapdh, were amplified and sequenced for Cercospora isolates previously investigated by Bakhshi et al. (2015a) and some related reference isolates investigated by Groenewald et al. (2013). Phylogenetic performance of the eight loci (ITS, tef1, act $A$, $c m d A$, his3, tub2, rpb2 and gapdh) were assessed based on the inter-/intraspecific distance ratio and clade recovery. With the final classification presented here, none of the genes we analysed provides an effective barcode on its own across the entire genus. However, gapdh emerged as a strong candidate for improved species delimitation in Cercospora and provides better insight, especially into species complexes. Groenewald et al. (2013) evaluated this gene in the Cercospora sp. $Q$ species complex and their results also showed high variation in this gene. The performance of gapdh in species delimitation has been also reported in other fungal groups, including Alternaria (Woudenberg et al. 2013) and the Colletotrichum gloeosporioides species complex (Weir et al. 2012). Additionally, when using the gapdh gene, $\mathrm{cmdA}$ sequences are crucial to distinguish some species of Cercospora. We therefore recommend gapdh as the gene for species delimitation in Cercospora. However, it needs to be combined with $c m d A$, tef1 and tub2 to obtain a robust species identification. In addition, data from the ITS, actA, rpb2, and his3, have been useful, and were at times necessary, to provide clear evidence of multi-gene phylogenetic concordance to separate cryptic species.

The amplification of gapdh with the available primers was not, however, easy, and we need to design new primer sets for gapdh in Cercospora derived from the sequences generated. On the other hand, lack of ex-type or reliable sequences in public databases is a serious problem in the accurate molecular identification of Cercospora species, and it is essential to also amplify at least the gapdh and tub2 genes for all of the reference isolates used by Groenewald et al. (2013) in the future.

One of the main goals of this project was to generate an eight-gene DNA dataset for species of the genus Cercospora. In this regard, one of the achievements of this research was that the sequencing of additional loci revealed new clades within some taxa which were found to actually represented a species complex (in the eight-gene phylogenetic tree) rather than a single species, while the five-gene phylogenetic tree (Bakhshi et al. 2015a) was unable to resolve them. The phylogenetic tree based on the combined eight-gene dataset resolved at least four, three, two, two and two wellsupported clades respectively within the species complexes C. cf. flagellaris, C. apii, C. beticola, C. armoraciae, and Cercospora sp. G.
Some of the species revealed by the eight-gene phylogeny in this study can be distinguished based on their morphology or host range. For example, as explained in the notes for $C$. uwebrauniana, characteristics of the conidia in this species, which clustered in the $C$. apii complex based on the five-gene phylogenetic tree (see Bakhshi et al. 2015a), are clearly distinguishable from those of $C$. apii. However, some species cannot be separated using morphological characters. For instance, the $C$. cf. flagellaris species complex included at least three distinct clades and there is considerable overlap between morphological features and host ranges of the clades 1,2 , and 3 . In addition, pursuant to high levels of intraspecific variation in these three clades, the distinction between these clades is only possible based on molecular data. It is conceivable that some members of these three clades represent new species, yet to be described. This is also true for the Cercospora sp. G species complex.

Another problem arises because many morphological features change according to the host plant and different weather conditions. Such differences in morphological characters under different conditions have also been seen in other groups of fungi, such as Colletotrichum species (Weir et al. 2012). Because we do not yet have access to sequence data of most species of the Cercospora, we have chosen to consider these clades as different clades of $C$. cf. flagellaris and Cercospora sp. G rather than introduce new species names. Recent molecular studies on the Cercospora species associated with Cercospora leaf blight and purple seed stain on soybean, have revealed several Cercospora species, including C. cf. flagellaris as one of the most important agents (Bakhshi et al. 2015a, Soares et al. 2015, Albu et al. 2016). In this regard, Guillin et al. (2017) studied the genetic entanglement between Cercospora species infecting soybean and provided evidence that revealed interspecific gene flow played a significant role in the evolutionary dynamics of Cercospora species. Taking into consideration the shared host range that exists between different clades of $C$. cf. flagellaris, our data also provide more support for this hypothesis.

Furthermore, we found that all of the isolates of $C$. apii obtained from Plantago lanceolata from different localities clustered in clade 2 of this species in the eight-gene phylogenetic tree. Additionally, isolates of $C$. beticola and $C$. apii which intermix with $P$. lanceolata, had a common allele in gapdh. Thus, it seems that the gapdh gene might play a role in pathogenicity or host range, and has the potential to reflect this phylogenetically; however, that remains to be tested.

This study emphasises the complex nature of the evolutionary pathways that have been traversed within the genus Cercospora. Speciation has taken place much more prolifically than had previously been suspected in this genus, and it seems likely that the C. apii sensu Crous \& Braun (2003) species complex is still rapidly evolving. The emergence of new species is doubtlessly encouraged by the opportunities for mixing gene pools that are provided by modern global agricultural practices, and indiscriminate use of fungicides combined with imperfect phytosanitary regulation.

The present study provides the first eight-gene phylogenetic overview of Cercospora species. We hope that this dataset will provide a stable platform to accommodate the numerous undescribed species that still await description, 
and the recollection and epitypification of already named species. Moreover, it seems that Cercospora should still be subjected to a more detailed analysis based on yet additional gene loci to provide a more vigorous phylogenetic basis for species delimitation.

\section{ACKNOWLEDGEMENTS}

We gratefully acknowledge the Iran National Science Foundation (INSF), Research Deputy of the Iranian Research Institute of Plant Protection, Agricultural Research, Education and Extension Organization (AREEO), Westerdijk Fungal Biodiversity Institute, Research Deputy of the University of Tabriz, and Studienstiftung für mykologische Systematik und Ökologie for financial support. We thank Mieke Starink-Willemse, for her invaluable technical support and assistance in sequencing of some of the isolates. Uwe Braun (Martin Luther University, Halle, Germany) is thanked for comments pertaining to typification of several of the Cercospora spp. studied here.

\section{REFERENCES}

Agrios GN (2005) Plant Pathology. $5^{\text {th }}$ edn. New York: Academic Press.

Albu S, Schneider R, Price P, Doyle V (2016) Cercospora cf. flagellaris and Cercospora cf. sigesbeckiae are associated with Cercospora leaf blight and purple seed stain on soybean in North America. Phytopathology 106: 1376-85.

Bakhshi M, Arzanlou M, Babai-Ahari A (2012a) Comprehensive check list of Cercosporoid fungi from Iran. Plant Pathology and Quarantine 2: 44-55.

Bakhshi M, Arzanlou M, Babai-Ahari A (2012b) Morphological and molecular characterization of Cercospora zebrina from black bindweed in Iran. Plant Pathology and Quarantine 2: 125-130.

Bakhshi M, Arzanlou M, Babai-ahari A, Groenewald JZ, Braun U, Crous PW (2015a) Application of the consolidated species concept to Cercospora spp. from Iran. Persoonia 34: 65-86.

Bakhshi M, Arzanlou M, Babai-Ahari A, Groenewald JZ, Crous PW (2015b) Is morphology in Cercospora a reliable reflection of generic affinity? Phytotaxa 213: 22-34.

Berlese AN (1888) Fungi Veneti novi vel critici. Malpighia 2: 241-250.

Braun U (2002) Miscellaneous notes on some micromycetes (II). Schlechtendalia 8: 33-38.

Braun U, Crous PW (2016) (2415) Proposal to conserve the name Cercospora (Ascomycota: Mycosphaerellaceae) with a conserved type. Taxon 65: 185

Braun U, Crous PW, Nakashima C (2014) Cercosporoid fungi (Mycosphaerellaceae) 2. Species on monocots (Acoraceae to Xyridaceae, excluding Poaceae). IMA Fungus 5: 203-390.

Braun U, Crous PW, Nakashima C (2015a) Cercosporoid fungi (Mycosphaerellaceae) 3. Species on monocots (Poaceae, true grasses). IMA Fungus 6: 25-97.

Braun U, Crous PW, Nakashima C (2015b) Cercosporoid fungi (Mycosphaerellaceae) 4. Species on dicots (Acanthaceae to Amaranthaceae). IMA Fungus 6: 373-469.

Braun U, Crous PW, Nakashima C (2016) Cercosporoid fungi (Mycosphaerellaceae) 5. Species on dicots (Anacardiaceae to Annonaceae). IMA Fungus 7: 161-216.
Braun U, Nakashima C, Crous PW (2013) Cercosporoid fungi (Mycosphaerellaceae) 1. Species on other fungi, Pteridophyta and Gymnospermae. IMA Fungus 4: 265-345.

Cabral A, Rego C, Nascimento T, Oliveira H, Groenewald JZ, Crous PW (2012) Multi-gene analysis and morphology reveal novel Ilyonectria species associated with black foot disease of grapevines. Fungal Biology 116: 62-80.

Chupp C (1954) A Monograph of the Fungus Genus Cercospora. Ithaca, NY: C. Chupp.

Crous PW, Aptroot A, Kang JC, Braun U, Wingfield MJ (2000) The genus Mycosphaerella and its anamorphs. Studies in Mycology 45: 107-121.

Crous PW, Braun U (2003) Mycosphaerella and its Anamorphs: 1. Names published in Cercospora and Passalora. [CBS Biodiversity Series no. 1.] Utrecht: Centraalbureau voor Schimmelcultures.

Crous PW, Braun U, Hunter GC, Wingfield MJ, Verkley GJM, et al. (2013) Phylogenetic lineages in Pseudocercospora. Studies in Mycology 75: 37-114.

Crous PW, Gams W, Stalpers JA, Robert V, Stegehuis G (2004a) MycoBank: an online initiative to launch mycology into the 21st century. Studies in Mycology 50: 19-22.

Crous PW, Groenewald JZ (2005) Hosts, species and genotypes: opinions versus data. Australasian Plant Pathology 34: 463-470.

Crous PW, Groenewald JZ, Mansilla JP, Hunter GC, Wingfield MJ (2004b) Phylogenetic reassessment of Mycosphaerella spp. and their anamorphs occurring on Eucalyptus. Studies in Mycology 50: 195-214.

Crous PW, Schoch CL, Hyde KD, Wood AR, Gueidan C, et al. (2009a) Phylogenetic lineages in the Capnodiales. Studies in Mycology 64: 17-47.

Crous PW, Summerell BA, Carnegie AJ, Wingfield MJ, Hunter GC, et al. (2009b) Unravelling Mycosphaerella: do you believe in genera? Persoonia 23: 99-118.

Damm U, Cannon PF, Woudenberg JHC, Johnston PR, Weir BS, et al. (2012) The Colletotrichum boninense species complex. Studies in Mycology 73: 1-36.

Deighton FC (1973) Studies on Cercospora and allied genera. IV. Cercosporella Sacc., Pseudocercosporella gen. nov. and Pseudocercosporidium gen. nov. Mycological Papers 133: 1-62.

Deighton FC (1979) Studies on Cercospora and allied genera VII. New species and redispositions. Mycological Papers 144: 1-156.

Deighton FC (1983) Studies on Cercospora and allied genera. VIII. Further notes on Cercoseptoria and some new species and redispositions. Mycological Papers 151: 1-13.

Drummond A, Ashton B, Buxton S, Cheung M, Cooper A, et al. (2012) Geneious. Version 5.6, http://www.geneious.com.

Ellis MB (1971) Dematiaceous Hyphomycetes. Kew: Commonwealth Mycological Institute.

Ellis MB (1976) More Dematiaceous Hyphomycetes. Kew: Commonwealth Mycological Institute.

Fresenius G (1863) Beiträge zur Mykologie. Vol. 3. Frankfurt: H.L. Brönner.

Gola G (1930) L'Erbario Micologico di P. A. Saccardo. Catalogo. Tipografia Editrice Antoniana, Padova.

Goodwin SB, Dunkle LD, Zismann VL (2001) Phylogenetic analysis of Cercospora and Mycosphaerella based on the internal transcribed spacer region of ribosomal DNA. Phytopathology 91: 648-658.

Groenewald JZ, Groenewald M, Braun U, Crous PW (2010) Cercospora speciation and host range. In: Cercospora Leaf Spot of Sugar Beet and related species (Lartey RT, Weiland J, 
Panella L, Crous PW \& Windels C, eds): 21-37. St Paul, MN: American Phytopathological Society Press.

Groenewald JZ, Nakashima C, Nishikawa J, Shin HD, Park JH, et al. (2013) Species concepts in Cercospora: spotting the weeds among the roses. Studies in Mycology 75: 115-170.

Groenewald M, Groenewald JZ, Braun U, Crous PW (2006) Host range of Cercospora apii and $C$. beticola and description of $C$. apiicola, a novel species from celery. Mycologia 98: 275-285.

Groenewald M, Groenewald JZ, Crous PW (2005) Distinct species exist within the Cercospora apii morphotype. Phytopathology 95: 951-959.

Guillin EA, de Oliveira LO, Grijalba PE, Gottlieb AM (2017) Genetic entanglement between Cercospora species associating soybean purple seed stain. Mycological Progress 16: 593-603.

Guatimosim E, Schwartsburd PB, Barreto RW, Crous PW (2017) Novel fungi from an ancient niche: cercosporoid and related sexual morphs on ferns. Persoonia 37: 106-41.

Hebert PDN, Cywinska A, Ball SL, Dewaard JR (2003) Biological identifications through DNA barcodes. Proceedings of the Royal Society of London B 270: 313-321.

Hesami S, Khodaparast S, Zare R (2012) New reports on Cercospora and Pseudocercospora from Guilan province. Rostaniha 13: 95-100.

Hsieh W-H, Goh T-K (1990) Cercospora and Similar Fungi from Taiwan. Taiwan, Taipei: Maw Chang Book Company.

Katoh K, Standley DM (2013) MAFFT multiple sequence alignment software version 7 : improvements in performance and usability. Molecular Biology and Evolution 30: 772-780.

Lall G, Gill HS, Munjal RL (1961) Some Cercospora species from India-V. Indian Phytopathology 14: 115-119.

Lartey R, Caesar-TonThat T, Caesar A, Shelver W, Sol N, Bergman J (2005) Safflower: a new host of Cercospora beticola. Plant Disease 89: 797-801.

Little S (1987) Cercospora malayensis. CMI Descriptions of Pathogenic Fungi and Bacteria 916: 1-2.

Liu YJ, Whelen S, Hall BD (1999) Phylogenetic relationships among ascomycetes: evidence from an RNA polymerse II subunit. Molecular Biology and Evolution 16: 1799-1808.

Lombard L, Crous PW, Wingfield BD, Wingfield MJ (2010) Species concepts in Calonectria (Cylindrocladium). Studies in Mycology 66: 1-13.

Maddison WP, Maddison DR (2011) Mesquite: a modular system for evolutionary analysis. Version 2.75. http://mesquiteproject.org.

Montegut J (1967) Étude systématique des principales espèces. Coton et Fibres Tropicales 22: 451.

Montenegro-Calderón JG, Martínez-Álvarez JA, Vieyra-Hernández MT, Rangel-Macías LI, Razzo-Soria T, et al. (2011) Molecular identification of two strains of Cercospora rodmanii isolated from water hyacinth present in Yuriria lagoon, Guanajuato, Mexico and identification of new hosts for several other strains. Fungal Biology 115: 1151-1162.

Myllys L, Stenroos S, Thell A (2002) New genes for phylogenetic studies of lichenized fungi: glyceraldehyde-3-phosphate dehydrogenase and beta-tubulin genes. Lichenologist 34: 237-246.

Narayan S, Kharwar RN, Singh RK (2001) Some novel taxa of hyphomycete genus Cercospora causing foliar spots in India. Indian Phytopathology 54: 351-357.

Nylander JAA (2004) MrModeltest. Version 2.0. Program distributed by the author. Evolutionary Biology Centre, Uppsala University, Uppsala, Sweden.
O'Donnell K, Cigelnik E (1997) Two divergent intragenomic rDNA ITS2 types within a monophyletic lineage of the fungus Fusarium are nonorthologous. Molecular Phylogenetics and Evolution 7: 103-116.

Pirnia M, Zare R, Zamanizadeh HR, Khodaparast A (2012) New records of cercosporoid hyphomycetes from Iran. Mycotaxon 120: 157-169.

Pollack FG (1987) An annotated compilation of Cercospora names. Mycological Memoirs 12: 1-212.

Pretorius MC, Crous PW, Groenewald JZ, Braun U (2003) Phylogeny of some cercosporoid fungi from Citrus. Sydowia 55: 286-305.

Pons N, Sutton BC (1996) Cercospora and similar fungi on Heliotropium weeds. Mycological Research 100: 815-820.

Quaedvlieg W, Binder M, Groenewald JZ, Summerell BA, Carnegie AJ, et al. (2014) Introducing the Consolidated Species Concept to resolve species in the Teratosphaeriaceae. Persoonia 33: 1-40.

Quaedvlieg W, Groenewald JZ, De Jesús Yáñez-Morales M, Crous PW (2012) DNA barcoding of Mycosphaerella species of quarantine importance to Europe. Persoonia 29: 101-115.

Quaedvlieg W, Kema G, Groenewald JZ, Verkley GJM, Seifbarghi $S$, et al. (2011) Zymoseptoria gen. nov.: a new genus to accommodate septoria-like species occurring on graminicolous hosts. Persoonia 26: 57-69.

Quaedvlieg W, Verkley GJM, Shin H-D, Barreto RW, Alfenas AC, et al. (2013) Sizing up Septoria. Studies in Mycology 75: 307-390.

Rannala B, Yang Z (1996) Probability distribution of molecular evolutionary trees: a new method of phylogenetic inference. Journal of Molecular Evolution 43: 304-311.

Rayner RW (1970) A Mycological Colour Chart. Kew: Commonwealth Mycological Institute.

Ronquist F, Teslenko M, Van der Mark P, Ayres DL, Darling A, et al. (2012) MrBayes 3.2: efficient Bayesian phylogenetic inference and model choice across a large model space. Systematic Biology 61: 539-542.

Saccardo PA (1880) Conspectus generum fungorum italiae inferiorum nempe ad Sphaeropsideae, Melanconieas et Hyphomyceteas pertinentium systemate sporologico dispositorum. Michelia 2: 1-38.

Schoch CL, Seifert KA, Huhndorf S, Robert V, Spouge JL, et al. (2012) Nuclear ribosomal internal transcribed spacer (ITS) region as a universal DNA barcode marker for Fungi. Proceedings of the National Academy of Sciences, USA 109: 6241-6246.

Soares APG, Guillin EA, Borges LL, Da Silva AC, De Almeida ÁM, et al. (2015) More Cercospora species infect soybeans across the Americas than meets the eye. PloS One 10: e0133495.

Solheim WG (1930) Morphological studies of the genus Cercospora. Illinois Biological Monographs 12: 1-85.

Stewart EL, Liu Z, Crous PW, Szabo LJ (1999) Phylogenetic relationships among some cercosporoid anamorphs of Mycosphaerella based on rDNA sequence analysis. Mycological Research 103: 1491-1499.

Stielow JB, Lévesque CA, Seifert KA, Meyer W, Irinyi L, et al. (2015) One fungus, which genes? Development and assessment of universal primers for potential secondary fungal DNA barcodes. Persoonia 35: 242-263.

Stukenbrock EH, Quaedvlieg W, Javan-Nikhah M, Zala M, Crous PW, McDonald BA (2012) Zymoseptoria ardabiliae and Z. pseudotritici, two progenitor species of the Septoria tritici leaf blotch fungus Z. tritici (synonym: Mycosphaerella graminicola). Mycologia 104: 1397-1407. 
Tamura K, Stecher G, Peterson D, Filipski A, Kumar S (2013) MEGA6: Molecular Evolutionary Genetics Analysis Version 6.0. Molecular Biology and Evolution 30: 2725-2729.

Tessmann DJ, Charudattan R, Kistler HC, Rosskopf EN (2001) A molecular characterization of Cercospora species pathogenic to water hyacinth and emendation of C. piaropi. Mycologia 93: 323-334.

Verkley GJM, Quaedvlieg W, Shin H-D, Crous PW (2013) A new approach to species delimitation in Septoria. Studies in Mycology 75: 213-305.
Videira SIR, Groenewald JZ, Nakashima C, Braun U, Barreto RW, et al. (2017) Mycosphaerellaceae - chaos or clarity? Studies in Mycology 87: 257-421.

Weir BS, Johnston PR, Damm U (2012) The Colletotrichum gloeosporioides species complex. Studies in Mycology 73: 115180.

Woudenberg JHC, Groenewald JZ, Binder M, Crous PW (2013) Alternaria redefined. Studies in Mycology 75: 171-212. 